\section{Pulmonary Pathology (including Mediastinal)}

1849 Loss of p16INK4A Expression Predicts Worse Outcome in Thymic Carcinoma

Scott W Aesif, Marie-Christine Aubry, Eunhee S Yi, Sarah M Jenkins, Grant Spears, Anja C Roden. Mayo Clinic, Rochester, MN.

Background: In many human cancers, abnormalities in genes and proteins that regulate the cell cycle have been demonstrated. For instance, p16 ${ }^{\mathrm{INK} 4 \mathrm{~A}}(\mathrm{p} 16)$, a tumor suppressor, inhibits cyclin D1-mediated phosphorylation of the retinoblastoma protein and ultimately leads to cell cycle blockade. In non-small cell lung cancer, low p16 expression has been associated with increased cyclin D1 expression and worse survival. In thymic carcinoma, studies showed loss of p16 expression in approximately two thirds of cases. However, the association of loss of p16 expression with cyclin D1 expression, prognosis, stage and clinical parameters has not been investigated in thymic carcinomas. Design: Thymic carcinomas (1963-2013) were reviewed by 3 thoracic pathologists who agreed on the diagnosis. Medical records were studied. Consecutive slides were stained for $\mathrm{p} 16$ and cyclin D1. Percent tumor cell staining (p16, cyclin D1) was scored as $0(<1 \%$ or negative $), 1+(1-25 \%$ tumor cells staining $), 2+(26-50 \%), 3+(51-75 \%)$, and $4+(>75 \%)$. Staining profiles were correlated with patient demographics, clinical presentation, pathologic staging (TNM and modified Masaoka), tumor subtype (WHO), tumor size, and overall (OS) and disease free survival (DFS).

Results: Thymic carcinomas from 28 patients $(16 \mathrm{men})$ with a median age of 51 years (range 18-80) were included. Cases with $\mathrm{p} 16$ expression in $<1 \%$ of tumor cells $(\mathrm{n}=14)$ were more likely to be of $\mathrm{M} 1$ stage $(8 / 14,67 \%)$ than cases with higher p16 expression $(\mathrm{n}=14)(3 / 14,21 \%)(\mathrm{p}=0.04)$. Follow-up was available in 27 cases with 2-year DFS of $58.3 \%$ (95\% CI: 39.5\%, 77.2\%) (Kaplan-Meier estimate). Cases with p16 expression in $<1 \%$ tumor cells had shorter 2-year DFS (30.8\% [95\% CI: $5.7 \%, 55.9 \%]$ vs $85.1 \%$ [ $95 \%$ CI: $66.0 \%, 100 \%]$ ) than cases with higher p16 expression (overall $\mathrm{p}=0.0009$ ). No significant correlations were observed with regard to the number of cyclin D1 positive cells; though, nuclear expression was observed in all cases $(1+, 6 / 28,21 \%$ vs $\geq 2+, 22 / 28,79 \%$ ).

Conclusions: Loss of 16 expression portends worse outcomes in patients with thymic carcinoma. These results suggest that $\mathrm{p} 16$ is a prognostic parameter and might be implicated in the tumorigenesis of thymic carcinomas. While expression of cyclin D1 was observed uniformly in thymic carcinomas, the significance of this observation is uncertain.

1850 Interobserver Variability in Assessing Sarcomatoid Components of Biphasic Malignant Mesothelioma (BMM): Implications for Outcome Measures

Vijayalakshmi Ananthanarayanan, Wickii Vigneswaran, Diana Kircheva, Qudsia Arif, Thomas Krausz, Aliya N Husain. Loyola University Medical Center, Maywood, IL; University of Chicago, Chicago, IL.

Background: Among pleural malignant mesotheliomas (MM), sarcomatoid mesotheliomas have the worst prognosis. Biphasic mesothelioma (BMM) histology, defined as mesotheliomas with at least $10 \%$ of either epithelioid or sarcomatoid components confers an intermediate prognosis. In this study, we investigated a cohort of surgically resected BMMs 1) to see if relative percentages of sarcomatoid components can prognostically stratify these cases and 2) to evaluate the extent of agreement between two pulmonary pathologists in assessing the percentages of the two histologic components.

Design: H\&E stained slides of 49 cases of pleural decortication/extended pleurectomy (39 males, 10 females) with a diagnosis of BMM were retrieved from the pathology database (2008-2013) and clinical data were tabulated. Two pathologists independently assessed the percentages of epithelioid and sarcomatoid components for these cases in a blinded fashion. Data were analysed using uni-variable and log-rank statistics. Results: The mean age of this cohort was 70.1 years. The median overall survival (OS) was 255.5 days. Adjuvant chemotherapy $(\mathrm{n}=28)$ was associated with an improvement in overall survival $(\mathrm{p}=0.0014)$. Median survival was 11.0 months in $\mathrm{BMM}$ cases with $<$ $50 \%$ sarcomatoid component and 5.4 months in cases with greater than $51 \%$ sarcomatoid component. The overall correlation between the two raters in estimation of sarcomatoid proportions was strong $(\mathrm{r}=0.728)$, while the intra-class correlation coefficient was 0.684 $(p<0.001)$ for each of the histologic components. Bland-Altman plot showed no bias in interobserver rating with increasing sarcomatoid percentages. However, three cases showed substantial differences in the scores between two raters.

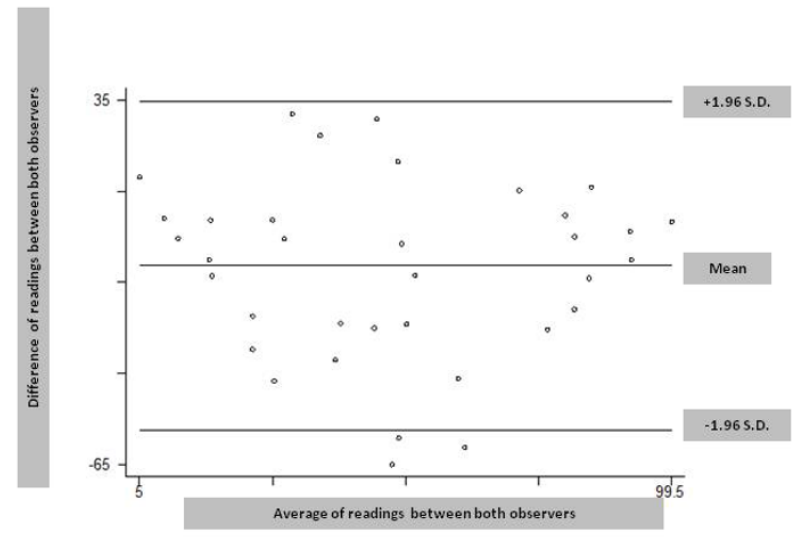

Conclusions: In this limited data set, we find that BMM cases with less than $50 \%$ sarcomatoid component have a survival advantage. Significant interobserver agreement is noted between two independent pathologists in sub-stratifying BMM cases.

1851 Programmed Death Ligand-1 (PD-L1) Expression in Operable Non-Small Cell Lung Cancer (NSCLC) by Immunohistochemical Analysis Sabina Babayeva, Artem Dyatlov, Ranjita Pallavi, Michael Fanucchi, Carmelo Puccio, Rocco Lafaro, Fouzia Shakil, John T Fallon, Minghao Zhong. New York Medical College, Valhalla, NY.

Background: Non-small cell lung (NSCLC) cancer is the leading cause of cancer-related deaths globally. Recently, FDA has granted approval for Programmed Death 1 ligand blocking antibody nivolumab in advanced NSCLC. Expression of Programmed Death Ligand-1 (PD-L1) has been showed as a predictive biomarker of anti-PD-1/PD-L1 therapies in some studies of advanced disease. However, pathological features associated with PD-L1 expression have not been studied in operable NSCLC. In this study, we evaluate pathological features associated with PD-L1 expression in operable NSCLC. Design: After IRB approval, the medical records of NSCLC patients with complete surgical resection between November 2012 and December 2014 were retrospectively reviewed at our institution. Tissue microarray (TMA) was constructed with 3 cores from each specimen. Immunohistochemical (IHC) stain was performed on Ventana system with anti-PD-L1 monoclonal antibody (E1L3N, 1:100 dilutions). e not been studied in operable NSCLC. In this study, we evaluate pathological features associated with PD-L1 expression in operable NSCLC.

Results: 73 cases of NSCLC (36 squamous cell carcinoma and 37 adenocarcinoma) were collected for this study. Patient characteristics were as follows: median age (range) 65 (51-85) years; female $68 \%$; and stage I/II/III/IV distribution: 58\%/31\%/7\%/4\%. $9(25 \%)$ SCC and 10(27\%) adenocarcinomas were positive for PD-L1. 66\% poorly differentiated (12/19) vs $33 \%$ well to moderately differentiated (7/19) Ca were positive for PD-L1. $40 \%$ higher stage $(=>\mathrm{pT} 2)(8 / 19)$ vs $60 \%$ lower stage $(11 / 19)(<\mathrm{pT} 2)$ Ca were positive for PD-L1. Interestingly, all (2/2) mucinous carcinoma are strongly positive for PD-L1. Conclusions: PD-L1 expression is positive in $\sim 25 \%$ of operable squamous cell carcinoma and adenocarcinoma. High grade and higher stage carcinoma are associated with higher rate of positivity for PD-L1. The few mucinous carcinomas are strongly positive for PD-L1. The correlation between PD-L1 expression and relapse free survival warrants further study.

1852 Programmed Death Ligand-1 (PD-L1) Expression in Squamous Cell Carcinoma from Different Anatomic Sites

Sabina Babayeva, Hongfeng Zhang, Michael Fanucchi, Rocco Lafaro, Yasmin Yusuf, John T Fallon, Minghao Zhong. New York Medical College, Valhalla, NY; Central Hospital of Wuhan, Wuhan, China.

Background: Programmed death-ligand 1 (PD-L1; also known as CD274 or B7-H1) represents a mechanism of immune escape for cancer. Therapeutics targeting this pathway gained accelerated approval from FDA for the treatment of several cancers, including lung squamous cell carcinoma. In this study, we would like to investigate PD-L1 expression in squamous cell carcinomas (SCC) from different anatomic sites. Design: After IRB approval, the medical records (including pathology reports) of squamous cell carcinoma patients with surgical resection were retrospectively reviewed at our institution between January 2012 and December 2014. 61 cases of squamous cell carcinoma from different sites (lung, head and neck, GYN and skin) were collected. Tissue microarray (TMA) was constructed with 3 cores from each specimen. Immunohistochemical (IHC) stain was performed on Ventana system with anti-PD-L1 monoclonal antibody (E1L3N, 1:100 dilution).

Results: We found that 25\% (9/36) of lung SCC, 67\% (4/6) of H\&N SCC, 29\% (2/7) of skin SCC and 25\% (4/12) cervical SCC were positive for PD-L1. In addition, PD-L1 expression was more likely associated with high grade carcinoma in different sites. Conclusions: PD-L1 expression is heterogeneous among squamous cell carcinomas from different sits, ranging $25 \%-67 \%$. High grade SCC was more likely to express PD-L1. PD-L1 immunotherapy may be effective for SCC from different sits, especially head \& Neck origin which had highest expression level. (2/7) of skin SCC and $25 \%$ (4/12) cervical SCC were positive for PD-L1. In addition, PD-L1 expression was more likely associated with high grade carcinoma in different sites. lution). 
1853 Cancer

Nike Beaubier, Valerica Mateescu, Julia A Elvin, Siraj M Ali, Elizabeth Morency, Ritu Nayar. Northwestern University, Chicago, IL; Foundation Medicine, Cambridge, MA. Background: Non-small cell lung cancer (NSCLC) has a 5-year survival of 30\% for stage II disease that drops to $1 \%$ for stage IV. Current National Comprehensive Cancer Network (NCCN) guidelines suggest targeted mutation analysis in EGFR and KRAS with translocation testing for ALK by fluorescent in situ hybridization (FISH) for advanced disease. This study examines the clinical utility of an extensive next generation sequencing (NGS) panel both to replace FISH testing and assist clinical trial enrollment. Design: DNA was extracted from 40 microns of FFPE sections from 28 cases of relapsed/metastatic lung adenocarcinoma. NGS was performed on hybridizationcaptured, adaptor ligation based libraries to a mean coverage depth of $>500 \mathrm{X}$ for up to 315 cancer-related genes plus 37 introns from 14 genes frequently rearranged in cancer. Genomic alterations (GA) included base substitutions, INDELs, copy number alterations and fusions/rearrangements. Clinically relevant GA were defined as GA linked to drugs on the market or under evaluation in mechanism driven clinical trials. Results: In the 28 lung adenocarcinoma samples 166 GA were found in 82 genes. Of these, $132(78 \%)$ were considered to be biologically or clinically relevant, including clinical trial eligibility. There were 17 GA in NCCN guideline genes, EGFR (16), KRAS (1). It should be noted that the percentage of KRAS mutations in this population is significantly lower than expected because most patients with known KRAS mutations were excluded from NGS testing. Adding targetable genes currently being investigated in late stage clinical trails, and suggested by the NCCN for testing, BRAF (7), MET (3), HER2 (3), RET (2), ROS1 (1), there are 33 GA (20\%) with direct clinical relevance. In addition, there are 16 TP53 GA. Of the 28 patients, $18(64 \%)$ have a GA in an NCCN required or suggested gene which could alter current treatment.

Conclusions: This study shows a high percentage of GA relevant to lung adenocarcinoma with 8 of 28 patients $(29 \%)$ having a currently targetable mutation in a late-stage clinical trial, which would not have been found by doing the minimum required NCCN testing of KRAS, EGFR and ALK. While costs must always be considered in healthcare decisions, NGS is justified by the low survival in advanced lung adenocarcinoma and high yield of mutations relevant to clinical trial enrollment. Also, being able to screen for translocations without FISH is advantageous since lung adenocarcinoma can harbor treatable translocations in three different genes.

\section{Clinical Detection of Resistance to EGFR Inhibitors in Lung} Cancers by Next Generation Sequncing

Deborah Belchis, Li-Hui Tseng, Lisa Haley, Peter B Illei, Christopher D Gocke, James $R$ Eshleman, Ming-Tseh Lin. Johns Hopkins School of Medicine, Baltimore, MD; National Taiwan Univeristy, Taipei, Taiwan.

Background: Getifinib or erlotinib are tyrosine kinase inhibitors approved by the Food and Drug Administration (FDA) for treatment of patients with EGFR-mutated metastatic lung cancers, but their benefit is limited by the development of acquired resistance. Detection of secondary resistant mutations may provide therapeutic or prognostic information.

Design: In this retrospective quality assessment study, we searched the $A K T, B R A F$, $E G F R, E R B B 2, K R A S, N R A S$, and PIK3CA genes by a clinically validated next generation sequencing (NGS) assay to identify secondary resistant mutations in 34 specimens submitted from 29 patients after getifinib or erlotinib therapy.

Results: NGS failed in $5(15 \%)$ specimens. Primary mutations were detected in 17 patients with an exon 19 deletion mutation, 7 patients with the p.L858R mutation, one patient with exon 20 insertion mutation and one patient with concomitant p.G719C and p.S768I mutations seen in both pre-treatment and post-treatment specimens. p.T790M was detected in $20(77 \%)$ of 26 patients. Mutant allele frequency was less than $10 \%$ and $20 \%$ in $10(44 \%)$ and $17(74 \%)$ of 23 p.T790M-mutaed specimens, respectively. An EGFR p.S768 V769delinsIL mutation was only seen in the post-treatment, but not the pre-treatment specimen. Additional p.K860I, p.G724S, and p.K754Q mutations were observed in 3 patients whose pre-treatment $E G F R$ mutations were documented at other institutes. PIK3CA mutations were detected in $8(31 \%)$ patients. Five of 8 PIK3CA mutations were outside of codons 542, 545, and 1047. A KRAS mutation was observed in one patient whose pre-treatment specimen was not available for testing. Transformation from adenocarcinoma to small cell carcinoma was observed in 4 $(14 \%)$ patients. Two or more resistant mutations were detected in 7 patients. Only 4 patients showed no EGFR p.790M mutation, PIK3CA mutation, KRAS mutation or transformation to small cell transformation.

Conclusions: NGS is a robust tool for detecting secondary resistant mutations. It demonstrates high analytic sensitivity, a broad reportable range, and

provides simultaneous detection of multiple resistant mutations. PIK3CA mutations appear to be a significant resistant mechanism. Upfront combination treatment targeting both EGFR and PIK3CA mutations may prevent development of resistance.

\section{Pulmonary Mucinous Adenocarcinoma- A Molecular Genetic Study} of 52 Cases

Jennifer M Boland, Joseph J Maleszewski, Benjamin R Kipp, Jason A Wampfler, Ping Yang, Eunhee S Yi. Mayo Clinic, Rochester, MN

Background: Invasive mucinous adenocarcinoma (IMA) is a recognized variant of lung adenocarcinoma in the 2015 WHO classification. IMA is defined as a tumor with at least $90 \%$ of cells showing classic goblet cell and columnar morphology, with abundant apical mucin and basal nuclei. Tumors that have a component of IMA but with more than $10 \%$ non-mucinous morphology are categorized as mixed IMA/non-mucinous adenocarcinoma (NMA). While IMA is known to frequently harbor KRAS mutations, other genetic abnormalities in these tumors are not as well defined, and it is not entirely clear if mixed IMA/NMAs share common mutations with pure IMAs.

Design: Slides from surgically treated lung adenocarcinomas $(n=763)$ were reviewed by 2 pulmonary pathologists. The diagnosis of IMA or mixed IMA/NMA was agreed upon by both pathologists. Sixty cases of IMA ( $7.9 \%)$ and 10 cases of mixed IMA/NMA $(1.3 \%)$ were identified. DNA was extracted from formalin-fixed paraffin embedded tumor samples and applied to the Ion AmpliSeq Cancer Hotspot Panel, which detects 207 mutations in 50 oncogenes and tumor suppressor genes, including $K R A S, E G F R$, $B R A F, E R B B 2, M E T, N R A S, R E T$, and TP53, among others. Testing was successful in 45 of 60 IMAs and 7 of 10 mixed IMA/NMAs.

Results: At least 1 mutation was identified in 40 of 45 IMAs (89\%) and 6 of 7 mixed IMA/NMAs (86\%). KRAS mutations were present in 33 of 45 IMAs $(73 \%)$ and 6 of 7 mixed IMA/NMAs (86\%). Fifteen of the total 39 KRAS mutations detected (38\%) occurred in combination with another mutation (7 STK11, 2 GNAS, 2 TP53, 2 CDKN2A, 1 PIK3CA, 1 with both $C D K N 2 A$ and $P I K 3 C A$ ). Other genes with mutations observed as sole abnormalities included 2 TP53, 1 BRAF (G464V), and 1 ERBB2. One tumor had both TP53 and GNAS mutations, and 1 had mutations in EGFR G796S, TP53, PTEN and PIK3CA. Six cases had no detectable mutations.

Conclusions: $K R A S$ mutations were by far the most commonly detected abnormality, present in $73 \%$ of IMAs and $86 \%$ of mixed IMA/NMAs. However, $38 \%$ of KRAS mutations occurred in conjunction with other mutations, especially STK11 mutations, which were present in 7 cases (13\%). TP53 mutations were present in 5 cases $(10 \%)$. Only 1 case had a mutation for which targeted therapy for lung cancer is available (EGFR mutation). Six cases did not have a detectable abnormality. Further analysis of these "negative" cases by single nucleotide polymorphism array for genomic copy number variations is ongoing.

1856 A Small Panel of microRNAs Effectively Separates Typical Carcinoids from Neuroendocrine Carcinomas and May Predict Outcome in Atypical Carcinoid Tumors

Yao-Tseng Chen, Xiaojing Yang, Kathrin Tyryshkin, Paula Ginter, Thomas Tuschl, Neil Renwick. Weill Cornell Medical College, New York, NY; Queen's University, Kingston, ON, Canada; Rockefeller University, New York, NY.

Background: Neuroendocrine tumors of the lung comprise a heterogeneous group of neoplasms with a wide variation in clinical behavior. We previously showed that miroRNA profiles readily separated typical carcinoid tumors (TC) from neuroendocrine carcinomas (NEC), including both small cell lung carcinomas (SCLC) and large cell neuroendocrine carcinoma (LCNEC). We have now validated these findings, identified their distinctive miRNA signatures, and tested this as a potential clinical predictor for atypical carcinoids (AC).

Design: microRNA sequencing data from a training set of 23 lung NET (4 TC, 9 AC, 5 SCLC and 5 LCNEC) were analyzed to identify miRNAs differentially expressed between carcinoid tumors (TC+AC) and NEC (SCLC+LCNEC) with high folddifferences (Kruskal-Wallis test). These miRNA candidates were tested by qRT-PCR on a validation set of 33 NET ( $10 \mathrm{TC}$ and $23 \mathrm{NEC}$ ) to develop a miRNA diagnostic tool for distinguishing TC vs. NEC. This molecular tool was applied to $6 \mathrm{AC}$ and correlated to the clinical outcome of these patients.

Results: A set of 32 miRNAs cleanly separated (TC+AC) from NEC in the training set. Most significantly, miR-196a showed higher expression in NEC $(\mathrm{P}=0.0001,3884$ fold-difference), whereas miR-129-2 showed higher expression in $(\mathrm{TC}+\mathrm{AC})(\mathrm{p}=0.0002$, 1035 fold-difference). miRNA cluster on chr.14q32, e.g. miR-487b and miR-136, also showed higher expression in (TC+AC), with 46 to 366 fold-differences.

miRNA qRT-PCR for miR-196a, $-129-2,-487 b$ and -136 were performed on the validation set of $10 \mathrm{TC}$ and 23 NEC (10 LCNEC, 6 primary SCLC and 7 metastatic SCLC). Results confirmed differential expression of all 4 miRNAs in TC vs. NEC, with p-value of $<0.0001,0.0004,0.0002$ and 0.0003 , respectively. The combined expression differences of these 4 miRNAs, calculated as delta-Ct $(129+136+487 b-196)$, had a median score of 7.40 for TC (range 1.51 to 14.89 ) and -13.48 for all NEC ( -4.49 to -21.24), with p-value $<0.0001$.

Six AC were then analyzed, and delta-Ct $(129+136+487 b-196)$ scores obtained ranged from 5.58 to -19.71 . Clinical follow-up showed the 2 cases with lowest scores in the NEC range (-19.71 and -10.09) to have recurred as metastatic SCLC. Other 4 cases had no recurrence or recurred as carcinoid tumors.

Conclusions: A small panel of 4 miRNAs can effectively separate typical carcinoids from neuroendocrine carcinomas of the lung. The expression of these miRNAs may also predict the clinical behavior of atypical carcinoids.

1857 Amplification of 3q26 and High Expression Levels of SOX2 Are Associated with a Favorable Prognosis in Patients with Lung Squamous Cell Carcinoma

Hoiwan H Cheung, Laura J Tafe. Geisel School of Medicine at Dartmouth, Hanover, NH; Dartmouth-Hitchcock Medical Center, Lebanon, NH

Background: Amplification of the $3 \mathrm{q} 26$ region is highly associated with lung squamous cell carcinoma (LUSC). While a likely contributing to tumororgenesis as $40-50 \%$ of LUSC harbor this alteration, the clinical and pathological outcomes stratified by 3 q 26 amplification status has not yet been described in LUSC. In this study we correlate relevant clinical data with 3q26 amplification to determine its prognostic utility in LUSC. Design: Clinical and pathological data from all 504 cases of LUSC were downloaded from The Cancer Genome Atlas (TCGA) and 3q26 amplification status was determined by mapping probes to the relevant region to calculate the $3 q 26$ copy number variation (CNV) for each tumor. Kaplan-Meier Survival Curves and Cox Proportional hazards regressions were used to determine the prognostic value of $3 q 26$ amplification. Survival 
analysis was also performed on patients stratified by SOX2 expression levels, a gene located within the $3 \mathrm{q} 26$ region of interest, in order to validate results. All statistical analyses were performed using the R 3.2.2 statistical package software

Results: 3q26 amplification was found to be highly favorably prognostic with regards to overall survival in LUSC patients (death Hazard Ratio $[\mathrm{HR}]=0.52, \mathrm{p}=.025$ ); survival differences persisted even after controlling for the pathologic tumor stage. Amplification of $3 \mathrm{q} 26$ also had increased tumor-free survival, (death $\mathrm{HR}=.48, \mathrm{p}=.13$, not significant). ECOG and Karnofsky scores, as well as rates of metastases did not differ significantly by 3 q26 amplification status. Patients with high levels of SOX2 expression also showed increased rates of survival (metastasis $\mathrm{HR}=.69, \mathrm{p}=.20$, not significant). Tumor-free survival did not differ by SOX2 expression levels.

\section{Overall survival by $3 q 26$ amplification}

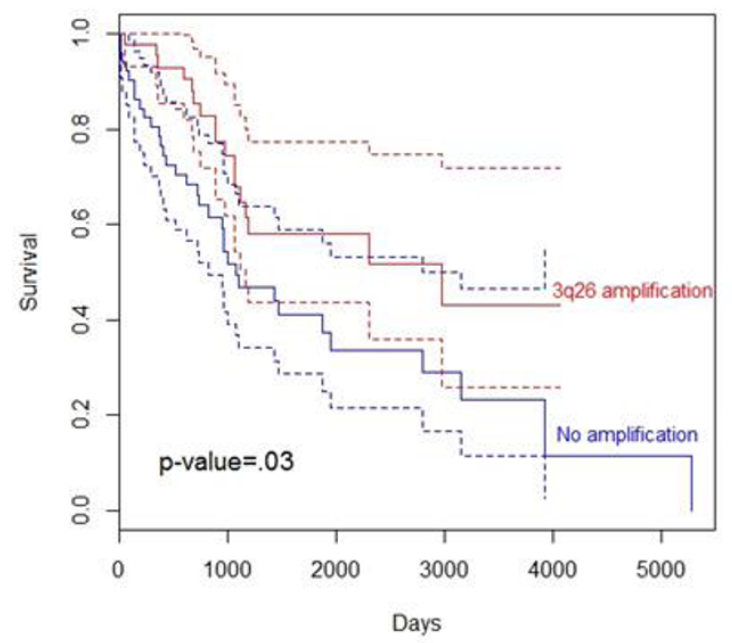

Conclusions: We show that $3 \mathrm{q} 26$ amplification confers an advantage in overall survival in LUSC. Similar directionality in survival advantage seen with increased SOX expression levels further validates our findings. Additional studies with long-term follow-up are needed to better determine the role of the $3 \mathrm{q} 26$ locus in the biological evolution and clinical significance in LUSC patients.

1858 Identification of a Novel Partner Gene, KIAA1217, Fused to RET: Functional Characterization and Inhibitor Sensitivity of Two Isoforms in Lung Adenocarcinoma

Yoon-La Choi, Mi-Sook Lee, Jhingook Kim. Samsung Medical Center, Seoul, Korea. Background: $R E T$ (rearranged during transfection) encodes a receptor tyrosine kinase and its fusion form is mainly known as an oncogenic driver factor in some types of cancer, including thyroid and lung. RET fusions were detected in approximately $1 \%$ of lung adenocarcinomas, but might partake as much as $6-19 \%$ of never-smokers without different genetic alteration. To date, six fusion partners in RET, which are KIF5B, CCDC6, CUX1, TRIM33, NCOA4 and KIAA1468, have all been reported in NSCLC. Design: Here, we report a novel RET fusion gene, KIAA1217, identified from a NSCLC patient. We also validated functional characteristics and inhibitor sensitivity with two isoforms of KIAA1217-RET constructs in vivo and in vitro.

Results: This novel KIAA1217-RET fusion gene wasgenerated through the combination of exon 11 of KIAA1217 and exon 11 of RET. Both isoforms of KIAA1217-RET fusion protein have led to increase in cell growth through activation of PI3K/AKT and ERK signaling pathways. In addition, it rendered invasive properties and subsequently was able to cause oncogenic transformation in lung cells. We also identified that cells expressing each isoform of KIAA1217-RET fusion protein were sensitive to vandetanib, which is a multiple RET kinase inhibitor, and treatment with vandetanib attenuated $\mathrm{PI} 3 \mathrm{~K} / \mathrm{AKT}$ and ERK signaling pathway.

Conclusions: In this study, we identified that KIAA1217-RET fusion is a novel oncogenic driver gene and gives rise to tumorigenicity in lung cells in both isoforms. This report provides experimental support for the use of RET inhibitors, and suggests its potential as a new diagnostic target and therapeutic options for the selected patients in NSCLC.

1859 The High Delta Ct Value of EGFR Exon 19 Deletion by PNA-Mediated PCR Clamping Can Predict Longer Progression-Free Survival in Non-Small Cell Lung Cancer

Yu-Deok Choi. Chonnam National University Medical School, Gwangju, Republic of Korea.

Background: Epidermal growth factor receptor (EGFR) mutation, such as deletions in exon 19 (19del) and point mutations in exon 21 (L858R) are reliable factors of response to EGFR tyrosine kinase inhibitors (TKIs) in non-small cell lung cancer (NSCLC). We investigated the quantification of EGFR mutation can predict progression-free survival (PFS) of EGFR-TKIs.

Design: We screened 555 cases for EGFR mutations using peptide nucleic acid (PNA)mediated clamping polymerase chain reaction (PCR) and found 156 single activating mutations (90 case of 19del and 66 cases of L858R in NSCLC). The efficiency of PCR clamping was determined by measuring the cycle threshold $(\mathrm{Ct})$ value and the EGFR quantification was determined by delta $\mathrm{Ct}(\Delta \mathrm{Ct})$ value which was calculated (wild type control Ct-sample $\mathrm{Ct}$ ).

Results: The median PFS of patients with $\Delta \mathrm{Ct} \geq 2.0$ (371 days, $95 \%$ confidence interval [CI] 336-406) had longer tendency as compared with $\Delta \mathrm{Ct} \leq 2.0(210$ days, $95 \% \mathrm{Cl}$ 53-367, $\mathrm{p}=0.280$ ). The median PFS of patients with $\Delta \mathrm{Ct} \geq 2.0$ was significantly longer than patients with $\Delta \mathrm{Ct}<2.0$ in cytology specimen ( 371 vs. 210 days, $\mathrm{p}=0.009$ ), but not in biopsy specimen $(\mathfrak{p}=0.453)$. In 19del subgroup, the median PFS of patients with $\Delta \mathrm{Ct} \geq 2.0$ (353 days, $95 \% \mathrm{CI} 203-503$ ) was significantly longer than patients with $\Delta \mathrm{Ct}<2.0(104$ days, $95 \% \mathrm{CI} 0-251, \mathrm{p}<0.001)$. And theses significant longer median PFS of patients with $\Delta \mathrm{Ct} \geq 2.0$ were shown both in biopsy ( $\mathrm{p}=0.028)$ and cytology specimen $(\mathrm{p}<0.001)$.

Conclusions: Quantification of EGFR mutation by $\triangle \mathrm{Ct}$ value of PNA-mediated PCR clamping can predict longer PFS of EGFR-TKIs in patients with 19del mutation regardless of specimen types.

1860 Mcl-1 Protein Expression and MCL1 Gene Amplification in Non Small Cell Lung Cancer (NSCLC)

Siddhartha Dalvi, Sungeun Kim, Christine E Sheehan, Jeffrey S Ross. Albany Medical College, Albany, NY.

Background: Myeloid cell leukemia 1 (Mcl-1) is a member of the B-cell lymphoma 2 (Bcl-2) family known to play a significant role in the regulation of apoptosis. Mcl-1 expression has been studied in NSCLC cell lines, but has not been previously evaluated as a prognostic factor in clinical samples. In the following study we assessed both Mcl-1 protein expression and $M C L 1$ gene amplification in NSCLC and compared the results with clinicopathologic variables.

Design: Formalin-fixed, paraffin embedded sections from 119 NSCLC, including 33 squamous cell carcinomas (SCC), 55 adenocarcinomas (AC), and 31 either pure bronchioloalveolar carcinomas (BAC) or AC with $\mathrm{BAC}$ features were immunostained by an automated method (Ventana/Roche, Tucson, AZ) with rabbit polyclonal Mcl-1 (clone S-19, Santa Cruz). Cytoplasmic (cMcl-1) immunoreactivity was scored based on intensity and percentage of positive tumor cells in both the tumor and adjacent benign epithelium in each case. Scoring was based on staining intensity (weak, moderate, intense) and percentage of positive cells (focal $<=10 \%$, regional $11-50 \%$, diffuse $>50 \%$ ). MCL1 gene amplification was determined by hybrid capture based comprehensive genomic profiling (CGP) on a separate cohort of 9,393 NSCLC samples.

Results: Intense diffuse cMcl-1 overexpression was noted in 35/119 (29\%) tumors overall and correlated with tumor type [ $52 \% \mathrm{BAC}$ vs $31 \% \mathrm{AC}$ vs. $6 \% \mathrm{SCC}, \mathrm{p}<0.0001]$, tumor grade [ $48 \%$ grade 1 vs $14 \%$ grade 2 vs $31 \%$ grade $3, p=0.007]$, small tumor size $[36 \%<=3.0 \mathrm{~cm}$ vs $16 \%>3.0 \mathrm{~cm}, \mathrm{p}=0.016]$, and lengthened survival within the BAC subgroup [ $[100 \%$ alive vs $42 \%$ expired, $p=0.018]$; while showing a trend toward correlation with non-recurrent disease overall $[32 \%$ non-recurrent vs $11 \%$ recurrent, $\mathrm{p}=0.072]$ and within the AC subgroup [ $33 \%$ non-recurrent vs $0 \%$ recurrent, $\mathrm{p}=0.092]$. MCL1 gene amplification was identified in $569(6 \%)$ of 9,393 NSCLC by CGP. On multivariate analysis, positive lymph node status $(\mathrm{p}<0.0001)$ independently predicted overall survival.

Conclusions: cMCL-1 overexpression appears to occur independently from $M C L 1$ gene amplification in NSCLC and correlates with bronchioloalveolar histologic type, lower tumor grade, smaller tumor size, non-recurrent disease, and increased survival. Continued studies are required to learn if cMCL-1 overexpression can be routinely used in surgical pathology practice for predicting prognosis for NSCLC cases.

1861 CTLA-4 Detection in Malignant Mesothelioma Tissues and in Effusions: Relevance for Immunotherapy

Franco Fedeli, Serena Varesano, Paola Ferro, Maria Cristiana Franceschini, Sandra Salvi, Simona Boccardo, Jean Louis Ravetti, Anna Morabito, Alessandro Valentino, Silvio Roncella, Maria Pia Pistillo. ASL5, La Spezia, Italy; IRCCS AOU San MartinoIST, Genoa, Italy.

Background: Malignant mesothelioma (MM) is a very aggressive tumor having a high mortality for absence of effective treatment. The immunotherapy might offer a new possibility of cure and in this contest, the anti-CTLA- $4 \mathrm{mAb}$ Tremelimumab represents a promising novel approach. Although CTLA-4 expression has been documented in a variety of tumors, in MM it has not been investigated so far. CTLA- 4 expressed by MM cells might bind to Tremelimumab and induce activation of cytotoxic immune cells as shown for melanoma cells (Pistillo MP et al. 2013). We investigated CTLA-4 expression in MM tissues and the possibility of releasing the soluble isoform (sCTLA-4) in pleural effusions $(\mathrm{PE})$ that might play a role in the immunotherapy as well

Design: CTLA-4 expression was detected by immunohistochemistry (IHC) in 29 formalin-fixed, paraffin-embedded MM tissues (15 epithelioid, 8 sarcomatoid, 6 others), using the anti-CTLA-4 mAb (14D3, eBioscence). CTLA-4 staining was classified by the immunoreactive score (negative, weak, intermediate and strong) based on both the percentage and intensity of positive cells. sCTLA- 4 was measured in $76 \mathrm{PE}$, including $45 \mathrm{MM}$ (28 epithelioid, 9 sarcomatoid, 8 others) and 31 pulmonary benign diseases (BNG), by using a specific ELISA kit (Bender MedSystems).

Results: Twenty eight out of 29 (96.5\%) MM tissues were found positive for CTLA-4 expression with variable intensity and proportion of positive cases. In particular, 4 out of 28 (14.3\%) CTLA-4-positivetissues showed strong expression, 18 (64.3\%) intermediate expression and $6(21.4 \%)$ weak expression.

The epithelioid MM showed the strong CTLA-4 expression in $20.0 \%$ of cases, whereas $73.3 \%$ of cases showed intermediate expression and $6.7 \%$ of cases were negative. In the sarcomatoid MM, CTLA-4 expression was strong in $12.5 \%$, intermediate in $75.0 \%$ and weak in $12.5 \%$ of cases. All the papillary and biphasic MM showed weak and intermediate expression, respectively. 
sCTLA-4 levels in PE were found $\geq 67 \mathrm{pg} / \mathrm{ml}$ (cut off) in 22/45 (48.9\%) MM samples and in 10/31 (32.2\%) BNG samples, but this difference did not reach statistical significance. Conclusions: We show that CTLA-4 expression can be detected in MM cells and variable levels of sCTLA-4 are present in PE, most likely released by the MM cells. Our findings may have important therapeutic implications for the current immunotherapy of MM based on CTLA-4 targeting.

1862 Inactivation of BRCA1 Associated Protein-1 (BAP1) in Mesothelioma. Immunohistochemical and Molecular Genetic Study of 94 Cases

Anna Felisiak-Golabek, Bartosz Wasag, Zengfeng Wang, Piotr Czapiewski, Wojciech Biernat, Jerzy Lasota, Markku Miettinen. National Cancer Institute, Bethesda, MD; Medical University of Gdansk, Gdansk, Poland.

Background: Malignant mesothelioma is a rare neoplasm that originates from serosal surface of pleural, peritoneal and pericardial cavities. Recent studies indicated loss of BRCA1-associated protein-1 (BAP1) in the familial and sporadic mesotheliomas. A wide variety of DNA mutations affecting BAP1 coding sequences was identified. Detection of these mutations may require extensive screening employing different molecular strategies. This study was undertaken to evaluate immunohistochemistry for BAP1 as the surrogate marker for loss of BAP1 function in malignant mesothelioma. Design: Ninety four mesotheliomas including 89 pleural, 4 peritoneal and 1 pericardial tumors were evaluated. The cohort consisted of 65 men and 29 women (mean age 60). Mesothelioma diagnosis was based on clinicopathologic and histological features, and expression of keratins, calretinin, and WT1-protein. BAP1 immunohistochemistry was performed using mouse mAB C-4 BAP1 antibody and Leica Bond-Max automatic immunostainer. DNA was extracted from formalin fixed paraffin embedded tumor samples in 66 cases. The multiplex ligand-dependent probe analysis (MLPA) was applied to detect large $B A P 1$ deletions, while PCR amplification followed by Sanger sequencing was used to screen $B A P 1$ for other DNA mutations.

Results: Lack of nuclear BAP1 expression was seen in 55 of $95(58 \%)$ analyzed cases. Tumors with losses included both epithelioid and sarcomatoid mesotheliomas. MLPA revealed large, up to several exon deletions in 8 tumors. Sanger sequencing identified four missense and two frame shift mutations leading to STOP codons and two in-frame deletions. Overall, $24 \%$ of tumors studied had BAP1 mutations. All tumors with large $B A P 1$ deletions or STOP codon mutations lack BAP1 expression. No such mutations were detected in mesotheliomas with nuclear BAP1 expression, although in two cases in-frame deletions were identified.

Conclusions: Screening for $B A P 1$ mutations in FFPE tissue is hampered by diversity of molecular changes and quality of DNA. Presence of BAP1 mutations such as large intragenic deletions, frame shift and missense mutations leading to STOP codons closely correlates with lack of nuclear BAP1 expression. Thus, immunohistochemistry is useful for assessing BAP1 functional status in malignant mesothelioma.

1863 ALK Rearragnement Testing by Immunohistochemistry and Fluorescent In Situ Hybridization in Lung Cancers: A Canadian Reference Testing Center's Routine Clinical Experience

Pierre Fiset, Jeffrey Tanguay, Scott Boerner, David M Hwang, Ming-Sound Tsao. University Health Network, Toronto, ON, Canada.

Background: A molecular subgroup of lung adenocarcinomas harbors rearrangements of the anaplastic lymphoma kinase (ALK) gene, the expression of which predict clinical response to ALK inhibitors such as crizotinib and ceritinib. The gold standard for detection of ALK rearrangements has been fluorescence in situ hybridization (FISH), however, immunohistochemistry (IHC) has gained acceptance as an alternate detection method, evidenced by the recent FDA approval of a stand-alone IHC test for ALK fusion-protein expression using the D5F3 antibody. We analyzed the clinical experience of using a validated ALK IHC assay with the $5 \mathrm{~A} 4$ antibody in routine practice of a large regional reference pathology laboratory.

Design: Data from lung cancer cases dating from January 2012 to September 2015 for which ALK IHC was performed were retrieved. IHC was performed using the $5 \mathrm{~A} 4$ antibody and a protocol optimized to detect ALK fusion proteins. IHC slides were reviewed by a thoracic pathologist or cytopathologist and reported as negative (no staining), equivocal (weak focal staining), or positive (diffuse moderate to strong staining). Cases showing equivocal or positive staining were validated by FISH breakapart probe testing.

Results: From 2012-15, we retrieved 5819 cases that had ALK testing. Of these, 120 (2.1\%) were reported as ALK positive, $164(2.8 \%)$ as equivocal, and $5535(95.1 \%)$ as negative for ALK staining. Of the ALK IHC positive cases, 10 (8.3\%) were inconclusive by FISH due to crush artifact, insufficient cells, or high background. Of the remaining 110 cases, 102 (92.7\%) were confirmed to have the ALK rearrangement by FISH. ALKIHC immunopositivity without rearrangement by FISH was seen in 8 cases (7.2\%). Of these, 3 cases showed additional ALK fusion signals seen on FISH suggesting either polysomy, and/or unbalanced structural rearrangements. Of the IHC equivocal cases, FISH was positive for $3(1.8 \%)$, negative for $145(88.4 \%)$, and inconclusive for $9(5.4 \%)$ due to insufficient tissue. Of the IHC equivocal cases, $77(47.0 \%)$ showed additional ALK fusion signals by FISH.

Conclusions: Our data show a low rate $(7.2 \%)$ of failure to detect ALK gene rearrangement by FISH for lung cancer cases that are ALK-IHC positive using the $5 \mathrm{~A} 4$ clone. Further characterization of this subset of ALK IHC positive/FISH-negative cases is needed to identify the genomic alteration(s) responsible for the discordances. Our results also suggest that FISH testing should be continued for detection of gene rearrangement in IHC-equivocal cases.
1864 Adipophilin Expression in Pulmonary Adenocarcinoma Is Associated with Poor Clinical Prognosis: Immunohistochemical Study of 328 Cases

Masakazu Fujimoto, Akihiko Yoshizawa, Shinji Sumiyoshi. Kyoto University Hospital, Kyoto, Japan; Wakayama Medical University, Wakayama, Japan; Kumamoto University Hospital, Kumamoto, Japan.

Background: Lipogenic pathway is up-regulated in proliferating cells including cancer. However, clinical impact of neoplastic steatogenesis remains unclear in lung cancers. Recent progress in immunostaining of PAT protein, which coats the surface of cytoplasmic lipid droplet, enabled the study of intracellular lipid using archived paraffin embedded tissue samples. In this report, we have evaluated the clinicopathological associations with the presence of intra-cytoplasmic lipid on lung adenocarcinomas (ADC), by analyzing the immunohistochemical expression of adipophilin (ADP), which is a member of PAT protein.

Design: We have immuno-stained ADP on tissue microarray composed of 328 primary lung ADC surgically resected at Kyoto University Hospital. We studied the correlation of the ADP immune-expression and clinical, molecular and survival data.

Results: Of the cohort, fifty-one (15.5\%) cases were ADP-positive. Positive vascular invasion, negative EGFR mutation and high Ki-67 index showed significant correlation with ADP-positive ADC, whereas no correlation with gender, age, smoking history, tumor stage, TTF-1 expression and KRAS mutation was found. Histologically, poorly differentiated, solid-predominant, enlarged nuclei with prominent nucleoli, and either abundant eosinophilic cytoplasm or abundant foamy cytoplasm, reminiscent of apocrine differentiation, were common in ADP-positive ADC. Regarding the prognostic significance, the ADP-positive ADC showed worse prognosis compared to the ADPnegatives in both disease free and overall survivals (log-rank test, $\mathrm{p}=0.047$ and 0.014 ). Univariate Cox regression analysis also indicated ADP expression as a poor prognostic marker in overall survival rate (HR: $2.0, \mathrm{p}=0.022$ ), but not in multivariate analysis. Conclusions: ADP, which is an indicator of cytoplasmic lipid droplets, is expressed in minority of lung ADC. ADP expression significantly correlates with high grade morphology, negative EGFR mutation, and is a negative prognostic indicator in lung $\mathrm{ADC}$. This indicates that a distinct phenotype with upregulation of lipogenic pathway exist in pulmonary ADC, which can be the target of lipogenesis inhibitor.

1865 Are There Biologic Relationships between Pulmonary Adenofibromas and Breast Fibroadenomas?

Nicola Fusco, Elena Guerini-Rocco, Davide Tosi, Marco Maggioni, Letterio Runza, Claudia Augello, Caterina Fumagalli, Davide Vacirca, Giulia Ercoli, Silvano Bosari, Massimo Barberis, Stefano Ferrero. Fondazione IRCSS Ca' Granda - Ospedale Maggiore Policlinico, Milan, Italy; European Institute of Oncology, Milan, Italy; University of Milan, Milan, Italy.

Background: Pulmonary adenofibroma is an extremely rare benign fibroepithelial neoplasm that could provide great diagnostic challenges to both clinicians and pathologists. This tumor with unknown phylogenesis histologically resembles fibroadenoma of the breast, which display recurrent somatic mutations in exon 2 of the mediator subunit 12 (MED12). Given the morphological similarity of these two biphasic neoplasms, here we sought to define whether adenofibromas of the lung would display similar immunoprofiles and somatic mutations compared to fibroadenomas of the breast. Design: Two adenofibromas of the lung (male $n=1$; female $n=1$ ) were retrieved from the authors' institutions and reviewed by four pathologists. Sections from formalinfixed paraffin-embedded (FFPE) tissue blocks were stained for estrogen receptor (ER), progesterone receptor (PR), thyroid transcription factor (TTF1), cytokeratin (CK) 7, CD99, and vimentin. DNA samples extracted from tumor and matched normal tissue sections were subjected to Sanger sequencing of MED12 exon 2.

Results: Histological analysis revealed a uniform and complex admixture of glandular and spindle cell components resembling the characteristic intracanalicular growth pattern observed in the majority of fibroadenomas occurring in young women. The epithelial component was arranged in "phyllodes-like" spaces lined by simple cuboidal to columnar epithelium and displayed strong positivity for TTF1 and CK7. The stromal component surrounding the epithelium was composed of bland spindle cells strongly positive for vimentin. ER was overexpressed in approximately $20 \%$ and $60 \%$ of the epithelial and mesenchymal cells, respectively, whereas the latter displayed strong and diffuse PR overexpression. None of the pulmonary adenofibromas harbored somatic mutations in the exon 2 of MED12 gene.

Conclusions: Our findings broaden the understanding of the biology underpinning pulmonary adenofibromas, suggesting that, in a way akin to fibroadenomas, ER and PR could play a part in their development. Furthermore, although MED12 exon 2 somatic mutations are likely to constitute the genomic driver of a subset of fibroepithelial tumors, including fibroadenomas, our results imply that pulmonary adenofibromas may be driven by other molecular alterations.

1866 High-Grade Components as the Second- or Third-Most Predominant Pattern Predict Poor Prognosis in pT1 Lung Adenocarcinoma Makoto Hamasaki, Tomoyuki Hida, Masayo Yoshimura, Sousei Abe, Akinori Iwasaki, Kazuki Nabeshima. Fukuoka University School of Medicine and Hospital, Fukuoka, Japan.

Background: Invasive lung adenocarcinoma is diagnosed based on the predominant histologic pattern according to the 2015 WHO classification, and histologic subtypes classified in that manner reflect prognosis. However, most adenocarcinomas are heterogenous and may contain high-grade components (HGCs) such as micropapillary, solid or cribriform patterns, as the second- or third-most predominant pattern. In this study, we investigated whether HGCs with less predominant patterns were associated with shorter survival even in small pT1 adenocarcinomas. 
Design: We reviewed 247 lung adenocarcinomas that had been surgically resected at the Department of Thoracic Surgery, Fukuoka University Hospital, between January 2006 and December 2009 and identified 121 pT1 adenocarcinomas. The tumors were classified according to the 2015 WHO classification. We defined micropapillary, solid, cribriform and partially solid/acinar patterns as HGCs. A partially solid/acinar pattern was defined as that in which solid and acinar structures are intermixed. The effects of the HGCs on overall survival were statistically analyzed.

Results: The $121 \mathrm{~T} 1$ adenocarcinomas included 13 adenocarcinomas in situ/minimally invasive adenocarcinoma (AIS/MIA, $10.7 \%$ ) and 58 papillary (PA,

47.9\%), 39 acinar (AA, 32.2\%), 4 micropapillary (MPA, 3.3\%), 3 solid (SA, 2.5\%) and 4 invasive mucinous (IMA, 3.3\%) adenocarcinomas. The 5-year overall survival rates for AIS/MIA, PA, AA, MPA, SA and IMA were 100\%, 82.4\%, 87.3\%, 25.0\%, 66.7\% and $75.0 \%$, respectively. HGCs as the second- or third-most predominant component were significantly associated with shorter survival in PA (hazard ratioo [HR] 2.98; 95\% confidence interval $[\mathrm{CI}]: 1.189-7.426 ; P=0.0188)$, while HGCs tended to correlate with shorter survival in AA (HR: $2.472 ; 95 \% \mathrm{CI}$ : $0.998-6.124 ; P=0.0506)$. HGCs as a minor component in PA, older age ( $\geq 75$ years) and vascular involvement significantly correlated with shorter survival by univariate analysis. However, multivariate analysis identified vascular involvement as the most significant predictor of poor prognosis. Conclusions: In PA and AA, HGCs included not as a predominant component but as a minor component could predict poor prognosis in pT1 lung adenocarcinoma.

1867 Squamous Metaplasia: Indicator of Acute Exacerbation and Poor Prognostic Factor in Usual Interstitial Pneumonia

Masatake Hara, Mikiko Hashisako, Yasuhiko Yamano, Takeshi Johkoh, Yasuhiro Kondoh, Hiroyuki Taniguchi, Junya Fukuoka. Nagasaki University School of Medicine, Nagasaki, Japan; Nagasaki University Graduate School of Biomedical Sciences, Nagasaki, Japan; Tosei General Hospital, Seto, Aichi, Japan; Kinki Central Hospital of Mutual Aid Association of Public School Teachers, Itami, Hyogo, Japan.

Background: Idiopathic Pulmonary Fibrosis is the most common disease among Idiopathic Interstitial Pneumonias (IIPs) and histologically shows Usual Interstitial Pneumonia pattern (UIP). One of the lethal factors in UIP is an event of acute exacerbation (AE) for which pathological indicators are unknown at present. We reviewed the cases of UIP to find the indicators which predict AE.

Design: We selected 91 cases which showed UIP of unknown cause. 12 histological findings were evaluated by two observers independently and graded semi-quantitatively into score of 0 to 3 . The consensus scores were obtained afterwards by the discussion which also includes another pathologist. Primary clinical data was also collected. The judgement of $\mathrm{AE}$ was given by experienced pulmonologists based on the guidelines. Association between the scores of each findings and AE was investigated using chisquare test and logistic regression. Survival analysis was performed by log rank test. Kaplan-Meier curves were also plotted.

Results: During the median follow-up of 40 months, 20 cases developed AE. 38 patients were dead (AE, 15; non-AE, 23). A median duration until the onset of AE is 497 days (4-2139 days). For clinical factors, only vital capacity was predictive for $\mathrm{AE}(\mathrm{p}<0.01)$. Histologically, squamous metaplasia showed positive association to the event of $\mathrm{AE}(\mathrm{p}=0.04)$.

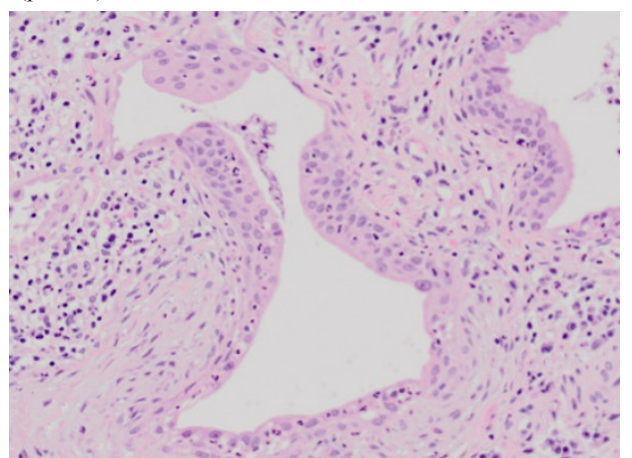

Fibroblastic foci, known as poor prognostic factor in UIP, did not associate with AE $(\mathrm{p}=0.3)$. Significant differences were noted between the prognosis and vital capacity $(\mathrm{p}<0.01)$ and squamous metaplasia $(\mathrm{p}=0.046)$.

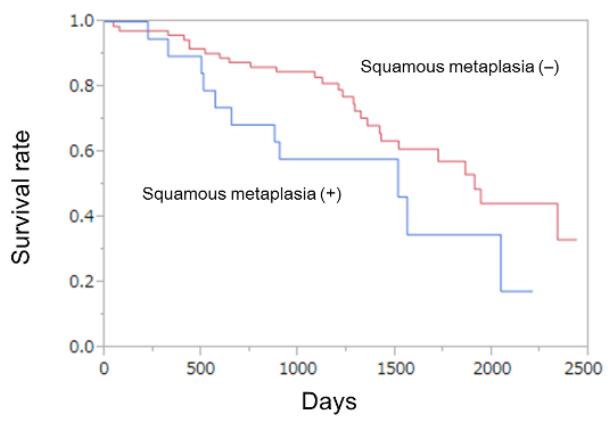

Conclusions: We found that presence of squamous metaplasia in surgical lung biopsy is an indicator of AE and a poor prognostic factor in UIP.
1868 Immunohistochemical Analysis of p14ARF, p15INK4B, p16INK4A, MTAP, and Their Combinations May Predict the Deletion Status of 9p21 in Malignant Pleural Mesothelioma

Tomoyuki Hida, Makoto Hamasaki, Shinji Matsumoto, Ayuko Sato, Tohru Tsujimura, Kunimitsu Kawahara, Yoshinao Oda, Kazuki Nabeshima. Fukuoka University School of Medicine and Hospital, Fukuoka, Japan; Graduate School of Medical Science, Kyushu University, Fukuoka, Japan; Hyogo College of Medicine, Nishinomiya, Hyogo, Japan; Osaka Prefectual Medical Center for Respiratory and Allergic Disease, Habikino-shi, Osaka, Japan.

Background: Differentiating malignant pleural mesothelioma (MPM) from reactive mesothelial hyperplasia (RMH) is still problematic. Homozygous deletion (HD) of $p 16^{I N K 4 A}(p 16)$, detected by fluorescence in situ hybridization (FISH), is a good marker for MPM in such a setting, but this method cannot be performed in all laboratories. Immunohistochemistry (IHC) is a more common, feasible technique, and a combined loss of methylthioadenosine phosphorylase (MTAP) and p16 expression has been proposed as a surrogate marker for $p 16 \mathrm{HD}$ in pancreatic and lung cancer. In this study, we investigated whether immunohistochemical detection of the loss of or decrease in expression of the products of genes located in the $9 \mathrm{p} 21$ region, such as $p 14^{A R F}, p 15^{I N K 4 B}$, p16, and MTAP, could predict the deletion status detected by $p 16$ FISH in MPM.

Design: Expression levels of $p 14^{A R F}, p 15^{I N K 4 B}, p 16$, and $M T A P$ were investigated immunohistochemically in 35 epithelioid MPM, and concordance between their results and the deletion status of the 9p21 locus as detected by FISH was analyzed statistically. Moreover, we examined combinations of the IHC expression levels of the gene products for concordance with $p 16$ FISH results.

Results: By IHC, loss or decreased expression of $p 14^{A R F}, p 15^{I N K A B}, p 16$, and MTAP were observed in $30 \%, 37 \%, 37 \%$, and $51 \%$ of MPM, respectively, while p16 HD was detected in $69 \%$ of MPM by FISH. Among the four genes, the results of MTAP IHC showed best accordance with those of $p 16$ FISH (kappa coefficient $[\mathrm{k}]=0.65$ ). Moreover, loss or decrease of $p 15^{I N K 4 B}-$ or-MTAP and that of $p 16-$ or-MTAP shown by IHC exhibited much better concordance $(\mathrm{k}=0.81$ and 0.76 , respectively). For predicting $p 16 \mathrm{HD}$ detected by FISH, loss or decrease of $p 15^{I N K 4 B}, p 16$, or MTAP, but not of $p 14^{A R F}$, showed $100 \%$ specificity.

Conclusions: Combinations of $p 15^{I N K 4 B}-$ or-MTAP and $p 16$-or-MTAP IHC can possibly detect most instances of $p 16$ HD in MPM, and thus could serve as useful ancillary IHC for discriminating MPM from RMH.

1869 Utility of BAP1 FISH and immunohistochemistry in Distinguishing between Mesothelioma and Reactive Mesothelial Proliferation. Comparison with Analysis of p16/CDKN2A

Kenzo Hiroshima, Di Wu, Mizue Hasegawa, Yasuo Sekine, Daisuke Ozaki, Toshikazu Yusa, Zhibin Gao, Ann E Walts, Alberto M Marchevsky, Yuji Tada, Hideaki Shimada, Masatoshi Tagawa. Tokyo Women's Medical University, Yachiyo, Chiba, Japan; Chiba Rosai Hospital, Ichihara, Chiba, Japan; Yuyao People's Hospital, Yuyao, Ningbo, China; Cedars-Sinai Medical Center, Los Angeles, CA; Chiba University, Chiba, Japan; Toho University, Tokyo, Japan; Chiba Cancer Center Research Institute, Chiba, Japan. Background: Distinction between mesothelioma and reactive mesothelial proliferation is sometimes difficult, especially when the biopsy specimen is small. However, correct diagnosis is crucial to patient care and compensation. It is reported that most mesotheliomas have deletion of $p 16 / C D K N 2 A$. Recently, loss of BAP1 expression has been reported in some mesotheliomas.

Design: FISH analysis of $p 16 / C D K N 2 A$ and immunohistochemistry (IHC) of BAP1 was performed on 47 surgical biopsies from histologically confirmed malignant mesotheliomas. BAP1 stain was also performed on 22 cell block samples (17 mesotheliomas and 5 reactive mesothelial proliferations). FISH analysis of $B A P 1$ gene was performed on tissue sections of 16 mesotheliomas.

Results: Homozygous deletion of $p 16 / C D K N 2 A$ was observed in $74.3 \%(26 / 35)$ of the mesotheliomas. There was a $100 \%$ concordance in CDKN2A deletion status between epithelioid and sarcomatoid components in biphasic mesotheliomas. BAP1 loss was observed in 50\% (15/30) of epithelioid mesotheliomas and 33.3\% (4/12) of biphasic mesotheliomas, but none $(0 / 5)$ of sarcomatoid mesotheliomas. BAP1 loss was observed in epithelioid component but not in sarcomatoid component of one of biphasic mesotheliomas. BAP1 was retained in reactive mesothelial proliferations. All of the mesotheliomas with BAP1 loss harbored homozygous deletion of $B A P 1$ gene. However, two mesotheliomas without BAP1 loss harbored homozygous deletion of $B A P 1$ gene. Homozygous deletion of $p 16 / C D K N 2 A$ and/or BAP1 loss was observed in $88.6 \%(31 / 35)$ of mesotheliomas. In cell blocks, homozygous deletion of $p 16 / C D K N 2 A$ was observed in $76.5 \%$ (13/17) and BAP1 loss was observed in $82.4 \%(14 / 17)$. Homozygous deletion of $p 16 / C D K N 2 A$ and/or BAP1 loss was observed in $88.2 \%(15 / 17)$ of cell blocks. Concordance of the results of homozygous deletion of $p 16 / C D K N 2 A$ and BAP1 loss between surgical biopsies and cell blocks of mesothelioma was good.

Conclusions: Some of mesotheliomas with homozygous deletion of BAP1 gene maintain BAP1 function. Because there is discrepancy of BAP1 loss in epithelioid and sarcomatoid component, BAP1 loss may be later event. BAP1 FISH and IHC is a good tool for the diagnosis of mesothelioma without $p 16 / C D K N 2 A$ deletion on surgical biopsies and on cell blocks. 
1870 Non-neoplastic Pulmonary Parenchymal Findings in Patients Undergoing Lung Resection for Mass Lesions

Yin Hung, Gary M Hunninghake, Rachel K Putman, Lynette M Sholl, Marina Vivero. Brigham and Women's Hospital, Boston, MA.

Background: Potentially significant non-neoplastic lung disease may be detected in patients undergoing resection for mass lesions, but the incidence of such findings is unknown. We determined the prevalence and characteristics of parenchymal findings in patients with benign and malignant lung nodules, aiming to increase awareness of findings that could potentially impact patient management.

Design: The study included 347 lung resections from 340 patients with benign or malignant mass lesions and available pre-surgical computed tomography obtained between January 2001 and July 2015. Histologic assessment of interstitial, pleural, airway, airspace, and vascular abnormalities was correlated with clinical and radiologic abnormalities. Non-neoplastic lung findings were categorized as significant if they could potentially alter pathologic diagnosis or patient management, explain reported symptoms, or account for radiologic findings.

Results: 195 women and 145 men (mean age 65.6, range 31.9 to 89.4) underwent resection for carcinomas $(256,74 \%)$ or benign nodules $(91,26 \%) .86(25 \%)$ specimens showed significant histologic parenchymal abnormalities, most commonly patchy granulomatous inflammation (27 patients, $7.8 \%$ ) and findings consistent with interstitial lung disease (ILD) (53, 15\%) including: smoking-related interstitial fibrosis (SRIF) (26, $7.5 \%)$, aspiration $(15,4.3 \%)$, undefined ILD $(9,2.6 \%)$, Langerhans cell histiocytosis (2), and sarcoidosis (1). Other findings included thrombotic angiopathy $(6,1.7 \%)$, misdiagnosis (2), small airway obliteration (1), aspiration and amyloidosis (1), and suspicious lymphoid infiltrates (4). Abnormalities were documented in 28 of 86 original pathology reports $(33 \%)$, of which $19(68 \%)$ were reviewed by pulmonary pathologists. SRIF and granulomas were the only abnormalities recognized by general pathologists. Clinical correlates to histologic abnormalities were present in 42 of 86 cases (49\%), 3 (4\%) of which had been diagnosed clinically prior to biopsy.

Conclusions: Potentially significant pulmonary parenchymal findings were present in $25 \%$ of patients undergoing resections for lung masses at our institution. Despite potential impact on histologic diagnosis and patient management, these findings often go unreported by general pathology practitioners. Increased awareness, education, and judicious use of subspecialty consultation may prevent misdiagnosis or misinterpretation and help improve patient management.

\section{Lung Adenocarcinomas with KRAS Mutations Are Biologically} Heterogeneous

Tiffany G Huynh, Justin F Gainor, Valentina Nardi, Mari Mino-Kenudson. Massachusetts General Hospital, Boston, MA.

Background: No effective targeted therapies are currently available for lung adenocarcinomas (ADC) with KRAS mutations, the most common oncogenic driver in NSCLC in western patients. We have reported the association of PD-L1 expression with KRAS mutations in ADC, suggesting that PD-1/PD-L1 blockade may be a treatment strategy for KRAS-mutated ADC (K-ADC). However, the majority of K-ADC do not exhibit PD-L1. In addition, multiple studies have shown heterogeneity in therapeutic responsiveness and biology in K-ADC. Thus, we evaluated a cohort of $139 \mathrm{~K}-\mathrm{ADC}$ with a few biomarkers to classify them into biologically relevant groups.

Design: p53, HNF4a, PD-L1 and CD8 IHC were performed on tissue microarray slides or a representative section of biopsy/cytology specimens. p53 nuclear expression was scored using a 3-tiered system $(0-2)$ with $0 \%$ and $50 \%$ cut-offs, and scores 0 and 2 were deemed abnormal as previously reported. Nuclear expression in $\geq 5 \%$ of tumor cells was considered positive for HNF4a. Membranous expression in $\geq 5 \%$ of tumor cells was deemed positive for PD-L1 expression. CD8+ tumor infiltrating lymphocytes (TILs) were scored using a 2 -tiered system $(0-1)$. The cohort was classified into p53 abnormal (KP), p53 normal \& HNF4a positive (KH) and p53 normal \& HNF4a negative (KN) groups. Clinicopathological variables, $\mathrm{PD}-\mathrm{L} 1$ expression, CD8+ TILs and progression free survival (PFS) were compared between the 3 groups.

Results: Of the 139 patients (62 stage I, 14 stage II, 20 stage III, and 43 stage IV), 73, 25 and 41 were classified into the KP, KH and KN groups, respectively. The KP group was associated with advanced stage (IV) $(41 \%, \mathrm{p}=0.023$ vs. KN), PD-L1 expression $(50 \%, \mathrm{p}=0.010$ vs. $\mathrm{KH}, \mathrm{p}=0.018$ vs. $\mathrm{KN})$ and increased CD $8+\mathrm{TILs}(29 \%, \mathrm{p}=0.011$ vs. $\mathrm{KH}, \mathrm{p}=0.008$ vs. $\mathrm{KN}$ ), while the $\mathrm{KH}$ group was associated with an invasive mucinous adenocarcinoma (IMA) morphology ( $48 \%, \mathrm{p}=0.0003$ vs. $\mathrm{KP}, \mathrm{p}=0.0021$ vs. $\mathrm{KN})$. As for survival, the $\mathrm{KN}$ group showed a trend towards adverse outcomes with the 3-year PFS being $57 \%, 74 \%$ and $46 \%$ for the $\mathrm{KP}, \mathrm{KH}$, and $\mathrm{KN}$ groups, respectively.

Conclusions: K-ADC appear to consist of biologically distinct groups. While those with $\mathrm{p} 53$ alterations more likely present at an advanced stage but may be amenable to the PD-1/PD-L1 axis blockade, HNF4a positive tumors more likely exhibit IMA morphology and follow a more indolent clinical course. Those with neither alteration may have addtional abnormalities such as LKB1 loss that is reportedly associated with poor survival in K-ADC. Additional study with LKB1 IHC is underway.

1872 Next Generation Sequencing: A Novel Approach to Distinguish Multifocal Primary Lung Adenocarcinomas from Intrapulmonary Metastases

Wendy Kadi, Snehal Patel, Jean Lopategui, Ann E Walts, Alberto M Marchevsky, Andy Pao, Tudor Mudalige, Angela Aguiluz. Cedars Sinai Medical Center, Los Angeles, CA. Background: Multifocal lung adenocarcinomas (ACAs) are difficult to stage and pose major problems in patient management. Whereas independent primary lung ACAs are staged individually and treated accordingly, contralateral pulmonary and extrapulmonary metastases are assigned to stage IV and treated with more aggressive therapy. Currently, this distinction is based on clinical, radiologic, and pathologic findings, but these approaches can yield equivocal and/or conflicting conclusions. This study explores the use of next generation sequencing (NGS) to distinguish independent primary lung ACAs from intrapulmonary metastases and facilitate more accurate staging of multiple lung ACAs.

Design: Samples of 40 formalin-fixed paraffin-embedded ACAs from 19 patients were selected from our institutional archive: 5 patients with synchronous bilateral lung ACAs (4 pairs, 1 trio), 5 patients with metachronous bilateral lung ACAs (5 pairs), and 9 patients with primary lung ACA/distant metastasis ( 8 pairs, 1 trio). Samples were analyzed using the Ion AmpliSeq Cancer Hotspot Panel v2 with aberrations filtered to include only non-synonymous amino acid changes in 2855 "hotspots" frequently mutated in 50 cancer-associated genes.

Results: All 5 synchronous bilateral ACA pairs/trios had entirely non-overlapping mutations. All 9 primary ACA/distant metastasis pairs/trio had identical or overlapping mutations. Of the 5 metachronous bilateral ACA pairs, the 2 suspected to represent primary/metastatic pairs based on clinicopathologic features had identical or overlapping mutations whereas the 3 suspected to be independent primaries had non-overlapping mutations.

Conclusions: Our findings suggest that metastatic and their paired lung primary ACAs harbor identical or overlapping mutations while independent primary lung ACAs display non-overlapping mutations. Future studies are needed to clinically validate NGS as a useful and objective tool in staging multifocal lung cancers as either independent primaries or intrapulmonary metastases.

\section{Prognostic Factors and Recurrence in Primary Lung Carcinoid} Tumor Treated at a Single Institution

Neda Kalhor, Guoping Xu, Junya Fujimoto, Francesco Stingo, Bonnie Glisson, Reza J Mehran, James C Yao, Cesar A Moran. MD Anderson Cancer Center, Houston, TX; Dali University, Yunnan, China.

Background: Primary Lung Carcinoid Tumors (PLCT) are uncommon neoplasms and formulating a clinically effective prognostic stratification is a challenge and to date, histologic grade remains the main predictive parameter established. This study aimed to identify predictors capable of selecting subgroups with higher risk of tumor progression and adverse overall survival.

Design: Patients with PLCT who underwent resection at MDACC between 1997 and 2012 with available clinical information and pathology material were retrospectively identified. Follow up ranged from 13-262 months. Histologic grading of the tumor was evaluated based on the current WHO criteria using mitosis and necrosis. Patients' clinical characteristics were extracted from the medical records. Tumor proliferation index was assessed using Ki67 immunohistochemistry with image-assisted quantitative analysis (Aperio) evaluating both hot spot (hs-Ki67) and mean index (m-Ki67). The primary end point was recurrence free survival (RFS), which was assessed using the Kaplan-Meier method. Univariable and multivariable Cox regression analysis was used to identify predictors for tumor recurrence.

Results: One hundred and twenty four patients ( $\mathrm{M}: \mathrm{F}=1: 1.88$, mean age: 52.4$)$ were identified including 101 typical carcinoids (TC) and 23 atypical carcinoids (AC). 74 patients were in pathologic stage I, 17 in stage II, 50 in stage III, and 3 in stage IV. Median follow up was 80.25 months (range, 13-262 months). m-Ki67 and hs-Ki67 ranged from 0.2-8.3 and 0.6-16 in AC, respectively. In TC, the m-Ki67 and hs-Ki67 varied from 0.03-8.7 and 0.1-14.6, respectively. 18 patients developed recurrent/metastatic disease. From univariable analysis, the major risk factors for tumor recurrence were Ki67 proliferation index (hs-Ki67: HR=1.29, $\mathrm{p}<0.0001 ; \mathrm{m}-\mathrm{Ki} 67: \mathrm{HR}=1.47, \mathrm{p}<0.0001$ ) and histologic grade $(\mathrm{HR}=0.12, \mathrm{p}=0.0001)$. When combined in a multivariable model, Ki67 hot spot index $(\mathrm{HR}=1.56, \mathrm{p}=0.0024)$ and histologic grade $(\mathrm{HR}=0.254, \mathrm{p}=0.0430)$ were independent predictors of recurrence after adjustment for age, stage and multifocality. Conclusions: This cohort provides supportive evidence for the use of Ki-67 hot spot index to identify patients with higher risk of tumor recurrence which can help the clinicians plan appropriate therapeutic strategies.

\section{Cell Cycle Progression Score Is a Promising Predictor of} Recurrence in Primary Lung Carcinoid Tumors

Neda Kalhor, Junya Fujimoto, Guoping Xu, Ryan Hoff, Placede Tiemeny, Susanne Wagner, Reza J Mehran, Cesar A Moran, Ignacio I Wistuba. MD Anderson Cancer Center, Houston, TX; Myriad Genetics, Inc., Salt Lake City, UT; Dali University, Yunnan, China.

Background: The prognosis of patients with primary lung carcinoid tumors (PLCT), including typical carcinoid (TC) and atypical carcinoid (AC), is difficult to predict. The cell cycle progression (CCP) score is a proliferation based expression profile that has been shown to provide significant prognostic information that can be utilized in stratification of NSCLC patients with higher risk of cancer-related death. The purpose of this study was to evaluate CCP score in pulmonary carcinoid tumors resected at a single institution.

Design: Formalin-fixed paraffin-embedded surgical tumor samples from 126 patients diagnosed with TC and AC who underwent surgical resection were analyzed for 31 proliferation genes by quantitative PCR. A CCP score was calculated from the mRNA expression levels of these genes. The primary endpoint was recurrence free survival (RFS). Cox proportional hazards regression analysis for the association of clinical variables and CCP score with RFS was performed.

Results: The study cohort consisted of 124 patients (M:F=1:1.88, mean age: 52.4) including $101 \mathrm{TC}$ and $23 \mathrm{AC}$. 74 patients were in pathologic stage I, 17 in stage II, 50 in stage III, and 3 in stage IV. Follow up ranged from 13 to 262 months. 18 patients developed recurrent/metastatic disease ( 7 TC and $11 \mathrm{AC}$ ), and 10 died of cancer-related ( $4 \mathrm{TC}$ and $6 \mathrm{AC}$ ). The CCP score was calculated in 93 patients (79 TC and $14 \mathrm{AC}$ ), including 13 recurrences. Histology ( $\mathrm{HR} 0.15,95 \% \mathrm{CI} 0.05-0.46$, $\mathrm{p}=0.0019)$, node status (HR 3.45, 95\%CI 1.11-10.66), $\mathrm{p}=0.029$ ), age (HR 1.06, 95\%CI 1.01-1.11, $\mathrm{p}=0.0086$ ), 
necrosis (HR 7.76, 95\%CI 2.26-26.6, $\mathrm{p}=0.0047$ ), pathological stage (HR 2.40, 95\% CI $1.33-4.33, \mathrm{p}=0.00035)$ and the CCP score (HR 3.15,95\% CI 1.77-5.59, $\mathrm{p}=0.00021$ ) were significant predictors of recurrence-free survival in univariate Cox $\mathrm{PH}$ models. In a multivariate model, pathological stage (HR 2.74, 95\%CI 1.17-6.42, $\mathrm{p}=0.016$ ) and the CCP score (HR per unit 2.04, 95\% CI 0.96-4.36, $\mathrm{p}=0.052$ ) were independent predictors of recurrence after adjustment for age, histology, necrosis and node status.

Conclusions: A significant correlation between CCP score and RFS is demonstrated in this cohort. A prospective CCP score may help in effective stratification of high risk primary lung carcinoid tumors that may require adjuvant therapy.

1875 Expression of P16 and Detection of Subtypes of HPV RNA in Primary Non-Small Cell Lung Cancers (NSCLC) and Their Potential Clinical Implication

Armen Khararjian, Xiao-Jun Ma, Zhen Zhang, Frederic Askin, Edward Gabrielson, Yuling Luo, Qing Kay Li. Johns Hopkins, Baltimore, MD; Advanced Cell Diagnostics, Hawyard, CA.

Background: P16 expression and HPV infections have been strongly linked to cervical and oropharyngeal squamous cell carcinomas (SQCC). However, there is controversy in the involvement of P16 and HPV in lung cancer. Studies have shown an association between both HPV16/18 infected lung tissues and SQCC and have also confirmed a higher HPV infection rate in SQCC. In this study, we examined P16 expression and performed HPV RNA probes on primary NSCLC positive for P16, and correlated levels of P16 with clinical outcomes in NSCLC patients.

Design: Primary NSCLC TMAs ( $0.6 \mathrm{~mm}$ in diameter, $3-4$ cores per case) were constructed using specimens retrieved from department archives. Included were 75 cases of ADC, 88 cases of SQCC, and 60 tumor-matched normal lung tissues. Immunohistochemistry (IHC) of P16 (clone INK4a/E6H4) was performed. Nuclear staining patterns were scored semi-quantitatively as 0-3+ (negative to strong positive). HPV RNA in situ hybridization (ISH) and HPV subtype detection were then performed. Clinical information was correlated.

Results: The overall frequency of high expression of P16 in ADCs and SQCCs was $50.7 \%$ and $35.2 \%$, respectively. HPV RNA was detected by ISH in both ADCs and SQCCs with variable P16 stains. HPV16/18 showed a similar detection rate between ADC (23.3\%) and SQCC (22.4\%); however, HPV31/33 showed higher detection rate of $16.4 \%$ in ADC than that of $7.1 \%$ in SQCC. In ADCs, patients with p16 positive tumors had smaller tumor size, earlier tumor stage, and longer survival, with a mean survival of 41.1 vs. 28.9 month $(\mathrm{P}=0.047)$. In SQCCs, a slightly worse survival for patients with $\mathrm{P} 16$ positive tumors (30.1 vs. 37.3 months), It did not reach statistical significance $(\mathrm{P}=0.382)$

Conclusions: Our data demonstrates that P16 is aberrantly expressed in a large subset of both ADC and SQCC. A subset of tumors with strong P16 staining were positive for HPV RNA by ISH. Elevated P16 levels appear to be correlated with better prognosis in patients with ADCs, but not of those with SQCCs. These findings suggest that molecular pathways that result in increased P16 levels might play different roles in these different subtypes of NSCLC. Detecting HPV RNA may suggest additional pathways in our understanding of HPV related tumorigenesis. While there has been previous controversy in the role HPV plays in NSCLC, our study shows support that some primary lung cancers are associated with HPV. This association may provide promise as the use of the HPV vaccine becomes more prevalent.

1876 Relationship between PD-L1 Expression and Epithelial Mesenchymal Transition in Adenocarcinoma of the Lung

Sehui Kim, Moon-Young Kim, Jaemoon Koh, Heounjeong Go, Yoon Kyung Jeon, Doo Hyun Chung. Seoul National University Hospital, Seoul, Republic of Korea; Seoul National University College of Medicine, Seoul, Republic of Korea; Asan Medical Center, Seoul, Republic of Korea.

Background: Immunotherapy targeting the PD-1/PD-L1 pathway has emerged as a promising therapeutic strategy for non-small cell lung cancer. However, mechanism underlying PD-L1 induction in NSCLC cells remains unclear. Epithelial-tomesenchymal transition (EMT) is important for cancer progression and involved in drug resistance and immune evasion. Previously we observed that pleomorphic carcinoma of the lung highly expressed PD-L1. Thus, we investigated the association of PD-L1 expression and EMT phenotype in pulmonary adenocarcinoma (pADC).

Design: Immunohistochemical study for PD-L1, E-cadherin, ZEB1, Snail, Slug and vimentin and EGFR mutation analysis were done in 508 cases with pADC. PD-L1 expression was scored from 0 to 3 , and cases with score of 2 or 3 were deemed PDL1-positive. H-score for E-cadherin, ZEB1, Snail, Slug and vimentin expression was estimated.

Results: PD-L1 expression was scored 0 in $14.6 \%(n=74), 1$ in $26.6 \%(n=135), 2$ in $50.8 \%(\mathrm{n}=258)$ and 3 in $8.1 \%(\mathrm{n}=41)$ of the 508 cases. Comparing PD-L1-negative versus PD-L1-positive group dichotomized, $\mathrm{H}$-scores of SNAIL and vimentin were higher in PD-L1-positive group (for SNAIL, 102.82 \pm 67.85 versus $142.05 \pm 66.06, p$ $<0.001$; for VT, $21.56 \pm 56.57$ versus $51.81 \pm 88.16($ mean \pm SD), $p<0.001)$. Cases were classified into epithelial, mesenchymal, epithelial-mesenchymal, and unspecified type according to the immunohistochemical results. PD-L1-positivity was significantly higher in patients with mesenchymal $(72.2 \%, 91 / 126)$ and epithelial-mesenchymal $(62.9 \%$, $88 / 140)$ type than those with epithelial $(48.9 \%, 44 / 90)$ and unspecified $(51.3 \%, 39 / 76)$ type $(p=0.002)$. This trends were maintained only in EGFR mutated pADC.

Conclusions: This study demonstrates that EMT phenotype is related with PD-L1 overexpression in pulmonary adenocarcinoma and that patients with pulmonary adenocarcinoma harboring EMT phenotype might benefit from PD-1/PD-L1 blockade.
1877 Favorable Prognostic Implications of FGFR1 Amplification and MYC Amplification or Overexpression in Resected Esophageal Squamous Cell Carcinoma

Dohee Kwon, Ji Y Yun, Bhumsuk Keam, Young T Kim, Yoon K Jeon. Seoul National University Hospital, Seoul National University College of Medicine, Seoul, Republic of Korea; The Tumor Immunity Medical Research Center, Cancer Research Center, Seoul National University College of Medicine, Seoul, Republic of Korea.

Background: Esophageal squamous cell carcinoma (ESCC) is one of the leading causes of cancer-related mortality. It has been recently demonstrated that MYC expression with amplified FGFR1 modulates oncogenic transformation and response to the FGFR1 inhibitors in pulmonary squamous cell carcinoma. Subset of ESCC also exhibits FGFR1 amplification, however, little has been known for the FGFR1 and MYC status.

Design: A total of 180 patients with resected ESCC were included excluding those who received neoadjuvant chemo- or radiotherapy and had distant metastasis. FGFRI and $M Y C$ were analyzed using fluorescence in situ hybridization and immunohistochemistry. Results: FGFR1 and MYC amplification was observed in $21.4 \%(37 / 173)$ and $54.2 \%$ (91/168) respectively in ESCCs. MYC high-amplification was positively correlated with MYC overexpression $(P<0.001)$. In contrast, $F G F R 1$ status was not correlated with $M Y C$ amplification or protein expression. MYC overexpression was significantly higher in ESCCs with pT1 $(P<0.001)$, stage I $(P<0.001)$ and no lymph node metastasis $(P=0.023)$. Survival analysis among 122 patients with ESCC who did not received adjuvant chemo-or radiotherapy revealed that $F G F R 1$ amplification was related with better disease-free survival (DFS) $(P=0.039)$ and overall survival (OS) $(P=0.024)$. MYC amplification was correlated with longer DFS $(P=0.028)$ and MYC expression also showed a significant association with prolonged DFS and OS $(P=0.032$ and $P=0.031$, respectively). Patients (17/119) with ESCC harboring combined $F G F R 1$ amplification and MYC overexpression exhibited better DFS $(P=0.024)$ and OS $(P=0.024)$. In contrast, patients who received adjuvant chemo- or radiotherapy exhibited no significant difference in prognosis according to the $F G F R 1$ or MYC status.

Conclusions: Our data suggested that FGFRl and $M Y C$ were variably amplified and overexpressed in ESCCs and might be related with favorable prognosis but not in those receiving adjuvant chemo- or radiotherapy. Role of targeted therapy against FGFR1 or MYC in ESCC remains to be explored.

1878 Myxoid Malignant Pleural Mesothelioma: A Rare Histological Subtype with Good Prognosis

Sylvie Lantuejoul, Nolwenn Le Stang, Arnaud Scherpereel, Jean-Claude Pairon, Francoise Galateau-Salle. Centre Léon Bérard, Lyon, France; Hôpital Calmette, CHRU de Lille, Lille, France; Université Paris-Est Creteil, Créteil, France.

Background: Malignant mesothelioma is a rare disease with a dismal prognosis and an overall survival, all histological subtypes included, of 12 months. The main histological subtypes of MM include epithelioid (EMM) type and non-epithelioid types (biphasic (BMM), sarcomatoid (SMM) and desmoplastic malignant mesothelioma (DMM)).

Design: The aim of our study was to decipher the histopathological characteristics of 150 myxoid malignant pleural mesothelioma (MPM) and to compare their clinical presentation andprognosis with other $4022 \mathrm{MPM}$, including $3236 \mathrm{EMM}, 418 \mathrm{BMM}$, 102 DMM and 268 SMM; all cases were retrieved between 1997 and 2015 from the French clinico-biological database of mesothelioma MESOPATH.

Results: We observed a female predominance in the myxoid MPM group (61 females ; 41\%) in comparison with other MPM $(26 \%$ of females $)(\mathrm{p}<0,0001)$ and patients were slightly younger ( $70 \mathrm{yrs} ; 42-87)$ than in the other MPM group (73 yrs ; 55-97) $(\mathrm{p}<0,0001)$. Sixty-seven percent of the myxoid MPM patients were asbestos-exposed, but less than in the control population $(\mathrm{p}=0,01)$. Clinically, patients with myxoid MPM presented with less thoracic pain and fatigue ( $\mathrm{p}=0,006$ and $\mathrm{p}=0,04$, respectively). By definition, myxoid MPM were characterized microscopically by a diffuse and predominant myxoid stroma and all were of EMM subtype, except 3 cases diagnosed as BMM. At immunohistochemistry, myxoid MPM more frequently expressed CK56 and calretinin than the other EMM from the control group $(\mathrm{p}=0.001$ and $\mathrm{p}=0.003$, respectively); there was no striking difference regarding P53, P16 and BAP1 expressions between the two populations. Median overall survival was 26 months for myxoid MPM patients versus 12 for the overall population, and the 3 -year survival rate was twice higher for myxoid MPM than for MME from the control group.

Conclusions: Myxoid MPM is a rare histological subtype of MPM, accounting for less than $2 \%$ of all MPM; it more frequently affects females, who are less asbestos-exposed. Myxoid MPM is worth to be recognized as it harbors a better prognosis than classical EMM and other MPM.

1879 Malignancy Risk for the Categories: Non-Diagnostic, Benign, Atypical, Suspicious and Malignant Used in the Categorization of Endobronchial Ultrasound Guided-Fine Needle Aspirates of Pulmonary Nodules

Lester Layfield, Leslie G Dodd, Benjamin Witt. University of Missouri, Columbia, MO; University of North Carolina, Chapel Hill, NC; University of Utah, Salt Lake City, UT. Background: Endobronchial ultrasound-guided fine-needle aspiration (EBUS-FNA) is frequently used for the workup of pulmonary nodules. While no universally accepted diagnostic classification exists, the Papanicolaou Society of Cytopathology has recommended the categories: Non-diagnostic, negative for malignancy, atypical, suspicious for malignancy, and malignant. Sensitivity and specificity for the EBUS technique have been documented, but little information is available for malignancy risk associated with these categories.

Design: Departments of Pathology records at the University of Utah and the University of North Carolina, Chapel Hill were searched for EBUS-FNAs of pulmonary nodules. 
Only cases with surgical follow-up were selected for inclusion in the study. Cytologic diagnosis and subsequent surgical diagnosis were correlated and malignancy risk calculated for each category. Sensitivity and specificity were also calculated.

Results: 155 EBUS-FNAs with surgical follow-up were obtained. Comparison of cytologic cases with and without surgical follow-up show no statistical differences in distribution of cytologic diagnoses. Risks of malignancy were: Non-diagnostic $40 \%$, negative for malignancy $24 \%$, atypical $54 \%$, suspicious for malignancy $82 \%$ and malignant $87 \%$. Sensitivity and specificity were $81 \%$ and $84 \%$ respectively for surgically confirmed cytologic diagnoses when indeterminate categories were excluded. Conclusions: The diagnostic categories are associated with increasing risk of malignancy running from negative for malignancy to malignant. The non-diagnostic category has a significant risk of malignancy. While the risk of malignancy for a negative diagnosis is substantial $(24 \%)$, it is significantly less than that associated with an atypical (54\%) or suspicious for malignancy ( $82 \%)$ diagnosis. A suspicious diagnosis carries a risk for malignancy essentially the same as a malignant diagnosis. The categories atypical and suspicious for malignancy appear to have substantially different risks for malignancy ( $54 \%$ vs. $82 \%)$. The atypical category has twice the risk of malignancy as a negative for malignancy diagnosis. This risk stratification may be useful for patient management.

1880 Bilateral Invasive Mucinous Adenocarcinomas (Former Mucinous Bronchioloalveolar Carcinoma): Molecular Analysis Confirms Intrapulmonary Spread Rather Than Separate Primary Tumors

Charles Leduc, Kay See Tan, Shaozhou Tian, Snjezana Dogan, Marc Ladanyi, William Travis, Natasha Rekhtman. MSKCC, NY, NY.

Background: Invasive mucinous adenocarcinoma (IMA) is characterized by abundant cytoplasmic mucin, lepidic growth pattern, and a tendency for multifocality and occasional bilaterality, presenting as consolidations or nodules radiologically. It is generally presumed that multifocal, particularly pneumonic-type IMA, represents aerogenous spread of a single tumor. However, we have encountered several patients with nodular IMAs, in whom contralateral disease developed after a long time-interval, raising the question of a separate primary tumor. To assess tumor clonality in this setting, we compared molecular profiles of matched bilateral IMAs.

Design: A 10-year retrospective search for bilateral IMAs identified 9 patients in whom both lesions had undergone molecular testing. Clinical, radiologic, pathologic features were reviewed. Metastasis from extrapulmonary sites was excluded clinically. Minimum molecular studies included analysis of common KRAS and EGFR mutations. In the majority of cases, mass-spectrometry genotyping for hotspot mutations in additional 6 genes (AKT1, BRAF, ERBB2, MAP2K1, NRAS, PIK3CA) was also performed.

Results: 5 patients had metachronous bilateral IMAs with a mean latency of $4.5 \mathrm{yrs}$ (10 mos- 8.5 yrs), while the remainder had synchronous bilateral disease at diagnosis. On CT scan, a discrete nodular/mass-forming appearance was more common (13/18, $72 \%)$ than a pneumonic-type appearance $(5 / 18,28 \%)$. None of the patients had nodal or distant metastases. In all 9 patients, molecular results in bilateral tumors were identical: KRAS G12V (3 pairs); KRAS G12D (2 pairs); KRAS G12C (1 pair); no mutation (3 pairs). Notably, 2 patients had nodular metachronous IMAs developing with a $\geq 8$ yr latency; tumors in both of these patients harbored matching KRAS mutations. Based on the expected mutation frequencies in IMA, the probability of 9 consecutive matched molecular results occurring by chance is $4.1 \times 10^{-6}$.

Conclusions: This is the first study to provide molecular support that bilateral IMAs represent intrapulmonary spread of a single tumor rather than separate primary tumors. They also show that tumor spread can manifest many years after diagnosis, and likely occurs via the aerogenous (large airway) route, as no nodal and distant metastases were observed. This study reinforces the unique biologic and clinical behavior of IMA, and confirms that multifocal IMA is distinct from multifocal, bilateral non-mucinous adenocarcinomas with lepidic pattern, which have been previously shown to generally represent separate primary tumors.

1881 Histopathologic Findings in the Lungs of Patients Treated with Extracorporeal Membrane Oxygenation

Hee Eun Lee, Eunhee S Yi, Anja C Roden. Mayo Cliinc, Rochester, MN.

Background: Extracorporeal membrane oxygenation (ECMO) is increasingly used to treat patients with respiratory and/or cardiac failure, to bridge to lung transplantation or in post-transplantation primary graft dysfunction. The outcome of ECMO is influenced by both the patient's underlying disease and ECMO-related complications. Histopathologic findings in the lungs of patients treated with ECMO have not been systematically studied.

Design: Institutional autopsy files (1995-2015) were queried for cases with a history of treatment with ECMO. All lung slides from each case were reviewed by a thoracic pathologist blinded to clinical findings. Histologic findings of interstitium, alveoli, airways, pleura, and vasculature were recorded. In some cases Verhoeff-Van Giesson stains $(\mathrm{n}=8)$ were obtained. Autopsy reports and medical records were reviewed. Results: Seventy-five patients ( $38 \mathrm{men}$ ) with a median age of 42 years (range, 0-77 years) were included. Histologic findings are summarized in table.

\begin{tabular}{|l|l|}
\hline Histologic findings & Patients (N=75), N (\%) \\
\hline Pulmonary hemorrhage & $47(62.7)$ \\
\hline Acute alveolar hemorrhage & 28 \\
\hline Alveolar hemosiderosis & 33 \\
\hline Acute lung injury & $39(52.0)$ \\
\hline Organizing pneumonia & 9 \\
\hline Diffuse alveolar damage & 13 \\
\hline Acute or organizing fibrinous pneumonia & 7 \\
\hline Acute lung injury, not otherwise specified & 1 \\
\hline Acute bronchopneumonia & 16 \\
\hline Aspiration pneumonia & 3 \\
\hline Thromboembolic disease & $35(46.7)$ \\
\hline Pulmonary artery (PA), recent & 25 \\
\hline PA, recanalizing & 10 \\
\hline Pulmonary vein (PV) & 11 \\
\hline Small vessels & 17 \\
\hline Hydrophilic polymer emboli & 2 \\
\hline Vascular changes & $27(36.0)$ \\
\hline PA, intimal fibrosis & 12 \\
\hline PA, medial hypertrophy & 2 \\
\hline PV, intimal fibrosis & 7 \\
\hline Intravascular calcifications & 17 \\
\hline Hemorrhagic infarct & $16(21.3)$ \\
\hline Others & \\
\hline Prominent alveolar foamy macrophages & 3 \\
\hline Alveolar or interstitial calcifications & 6 \\
\hline Fibrinous pleuritis & 19 \\
\hline Co & \\
\hline
\end{tabular}

Conclusions: Most common histologic findings in autopsy lungs of patients treated with ECMO included acute lung injury pattern, pulmonary hemorrhage, hemorrhagic infarct, thromboembolic disease, and vascular changes. While some findings might be associated with the patients underlying disease, many, including pulmonary hemorrhage, hemorrhagic infarct, thromboembolic disease and, at least in some patients, acute lung injury are likely related to ECMO. These findings will provide a better understanding of ECMO-related lung disease and might help to prevent and/or treat ECMO-related lung diseases.

\section{Caveolin-1 Enhances Brain Metastasis in Lung Cancer, Associated} with SNAIL

Jae-Hyuk Lee, Kyung-Hwa Lee, Kyung-Sub Moon, Ji Shin Lee, Chan Choi. Chonnam National University Hwasun Hospital, Jellanam-do, Republic of Korea.

Background: Caveolin-1 (Cav-1) has been reported to play an important role in the development of a variety of human cancers. We aimed to investigate an association between Cav-1 expression and lung cancer progression in the context of brain metastasis (BM).

Design: Cav-1 expression was investigated in a series of $102 \mathrm{BM}$ samples and paired 49 primary lung cancer samples, and unpaired 162 primary samples with (63 cases) or without (99 cases) metastasis to distant organs other than the brain. Lung cancer cell lines were used for in vitro functional study.

Results: High Cav-1 expression in tumor cells was observed in $52 \%$ (38 out of 73) of squamous cell carcinoma (SQC), and 33\% (45/138) of non-squamous cell carcinoma (non-SQC) $(P=0.006)$. While patients with high Cav-1 expression in primary lung nonSQC had a shorter overall survival than the patients with low Cav-1 expression (60.7 months vs. 69.5 months, $P=0.083$ ), Cav-1 expression level did not affect the survival diffrences in patients with primary lung SQC $(P=0.978)$. However, the proportion of high Cav-1 expression cases in SQC was increased after BM in both paired and non-paired samples of lung primary and BM ( $53 \%$ vs. $84 \%, P=0.034 ; 52 \%$ vs. $78 \%, P=0.020)$. In vitro assays revealed that Cav-1 knockdown inhibited invasion and migration of lung cancer cells. Cav-1 knockdown was consistently associated with SNAIL downregulation. This finding was also supported by that SNAIL expression increased along with Cav-1 expression in BM samples of SQC.

Conclusions: $\mathrm{Cav}-1$ is believed to play an important role in BM processes of lung cancer, depending on histotypes. The mechanism is possibly linked with SNAIL regulation.

1883 Neuroendocrine Markers Possible Predictive Factors for Survival in Small Cell Lung Carcinoma

James Lee, Zahra Maleki. Johns Hopkins Hospital, Baltimore, MD.

Background: Small cell carcinoma comprises $\sim 15-20 \%$ of lung carcinomas and usually presents with a hilar mass and disseminated disease. It has a poor prognosis, and most patients are not surgical candidates, so patients are treated with chemotherapy and/or radiation. Few markers are available for prognostication of outcome.

Design: From 2008 to 2010, 65 cases of small cell lung cancer were evaluated for synaptophisin and chromogranin expressivity. Expression was categorized as diffusely positive, focal positive, or negative. The time to death from diagnosis was calculated for each category.

Results: Out of these specimens, it was determined that when synaptophysin, and chromogranin were each individually positive, the mean time to death from diagnosis was 407 days (mean age 64.6 years, $\mathrm{N}=22$ ) and 440 days (mean age 61.7 years, $\mathrm{N}=9$ ), 
respectively. This increased to 560 days (mean age 64.6 years, $\mathrm{N}=10$ ) if both of them together were either diffusely positive or just partially positive. But if synaptophysin was positive and chromogranin was negative, the mean time to death from diagnosis increased to 319 days (mean age 64.3 years, $\mathrm{N}=8$ ). When synaptophysin and chromogranin were negative, the mean time to death from diagnosis was 237 days (mean age 65.8 years, $\mathrm{N}=5$ ) and 288 days (mean age 64.9 years, $\mathrm{N}=14$ ), respectively. [table1] Conclusions: Loss of synaptophysin and chromogranin expression did not significantly alter the mean age at which small cell lung cancer presents. However, loss of these immunomarkers portended a shorter survival time for the patients involved.

\section{Primary Mediastinal Germ Cell Tumors: A Clinicopathologic Study} of 32 Cases

Liping Liu, Rafael E Jimenez, John C Cheville, Marie-Christine Aubry, Anja C Roden. Mayo Clinic, Rochester, MN.

Background: Primary mediastinal germ cell tumors (PMGCT) are rare; data is sparse. The diagnosis is critical because patients are generally younger, potentially curable and require different treatment than other mediastinal tumors. Our study details morphologic diagnoses, treatment and outcomes of PMGCT.

Design: Institutional database was searched for GCT in the mediastinum (1995-2015). Metastases were excluded. All cases were reviewed.

Results: 32 PMGCT (24 men) (median age of 31 yrs [range, 2d-67yrs]) included teratomas (15 mature, 1 immature), 11 mixed PMGCT and 5 seminomas. Sarcomatous transformation (ST) was present in 2 (of 32) PMGCT including 1 mixed PMGCT and 1 mature teratoma. 4 patients with mixed PMGCT including the one with ST died of local disease. 3 patients with mature teratoma, including the one with ST developed recurrences, only the later also had distant disease. Clinical presentation, treatments, and f/u data are summarized in table.

\begin{tabular}{|c|c|c|c|c|c|}
\hline & $\begin{array}{l}\text { Mature } \\
\text { teratoma } \\
(\mathrm{n}=14)\end{array}$ & $\begin{array}{l}\text { Mature teratoma } \\
\text { with sarcomatous } \\
\text { transformation }(\mathrm{n}=1)\end{array}$ & $\begin{array}{l}\text { Immature } \\
\text { teratoma } \\
(\mathrm{n}=1)\end{array}$ & $\begin{array}{l}\text { Seminoma } \\
(\mathrm{n}=5)\end{array}$ & \begin{tabular}{|l|} 
Mixed \\
PMGCT \\
$(\mathrm{n}=11)$ \\
\end{tabular} \\
\hline Chest pain & $3(21)^{a}$ & $1(100)$ & $1(100)$ & 0 & $6(55)$ \\
\hline Cough & $1(7)$ & 0 & 0 & $1(20)$ & $5(45)$ \\
\hline $\begin{array}{l}\text { Shortness of } \\
\text { breath }\end{array}$ & $4(29)$ & 0 & 0 & $1(20)$ & $4(36)$ \\
\hline Weight loss & $1(7)$ & $1(100)$ & 0 & $1(20)$ & $3(27)$ \\
\hline SVC syndrome & $1(7)$ & 0 & 0 & 0 & $1(9)$ \\
\hline Asymptomatic & $6(43)$ & 0 & 0 & $2(40)$ & 0 \\
\hline $\begin{array}{l}\text { Tumor size }(\mathrm{cm}) \text {, } \\
\text { median (range) }\end{array}$ & $8.5(5-17)$ & 8 & unknown & $\begin{array}{l}10(2.1- \\
14.5)\end{array}$ & $7(4-21)$ \\
\hline $\begin{array}{l}\hat{I}^{2} \mathrm{HCG}(\mathrm{IU} / \mathrm{L}) \text {, } \\
\text { median (range), } \\
\text { normal<0.5 }\end{array}$ & $\begin{array}{l}0.5(0.2- \\
0.6)\end{array}$ & 1.1 & $<0.5$ & $\begin{array}{l}2.3(0.7- \\
59)\end{array}$ & $\begin{array}{l}196(<0.5- \\
83,000)\end{array}$ \\
\hline $\begin{array}{l}\text { AFP }(\mathrm{ng} / \mathrm{mL}), \\
\text { median (range), } \\
\text { normal }<6\end{array}$ & $\begin{array}{l}1.8(1.4- \\
5.3)\end{array}$ & 257 & 0.8 & $\begin{array}{l}3.4(1.1- \\
3.9)\end{array}$ & $\begin{array}{l}1,115(5.1- \\
12,726)\end{array}$ \\
\hline \multicolumn{6}{|l|}{ Resection } \\
\hline Complete & $13(93)$ & $1(100)$ & $1(100)$ & $2(40)$ & $8(73)$ \\
\hline Incomplete & $1(7)$ & 0 & 0 & $1(20)$ & $2(18)$ \\
\hline Biopsy & 0 & 0 & 0 & $2(40)$ & $1(9)$ \\
\hline $\begin{array}{l}\text { Neoadjuvant } \\
\text { therapy }\end{array}$ & $1(7)$ & $1(100)$ & 0 & $2(40)$ & $9(82)$ \\
\hline Adjuvant therapy & 0 & 0 & 0 & $2(40)$ & $6(55)$ \\
\hline $\begin{array}{l}\text { Follow up (yrs), } \\
\text { median (range) }\end{array}$ & $\begin{array}{l}0.8(0- \\
19.4)\end{array}$ & 16 & 2.1 & $1(0.1-9.9)$ & \begin{tabular}{|l|}
$4.7(0.6-$ \\
$22.3)$
\end{tabular} \\
\hline Died of disease & 0 & 0 & 0 & 0 & $4(36)$ \\
\hline
\end{tabular}

a\# of patients (column \%) within each diagnostic category

Conclusions: In our experience, patients with mixed PMGCT have the worst outcome compared to patients with teratoma and seminoma. Furthermore, their demise is the result of advanced locoregional rather than systemic disease. Accurate classification is critical to ensure appropriate treatment and to assess risk of progression.

1885 Two Plasma MicroRNA Panels for Diagnosis and Subtype Discrimination of Lung Cancer

Shaohua Lu, Hui Kong, Yingyong Hou, Hongguang Zhu, Chunxue Bai. Zhongshan Hospital, Fudan University, Shanghai, China; Fudan University, Shanghai, China. Background: Early and accurate diagnosis of lung cancer is crucial for effective treatment. This study aimed to identify plasma microRNAs for diagnosis of lung cancer and for further discrimination of small cell lung cancer (SCLC) from non-small cell lung cancer(NSCLC).

Design: Plasma microRNA expression was investigated using three independent cohorts including 1132 participants recruited between October 2008 and September 2014 from five medical centers. The subjects were healthy individuals and patients with NSCLC or SCLC. Microarrays were used to screen 723 human microRNAs in 106 plasma samples for candidate selection. Quantitative reverse-transcriptase PCR was applied to evaluate the expression of selected microRNAs. Two logistic regression models were constructed based on a training cohort $(\mathrm{n}=565)$ and then validated using an independent cohort $(\mathrm{n}=461)$. The area under the receiver operating characteristic curve $(\mathrm{AUC})$ was used to evaluate diagnostic accuracy.

Results: Plasma panel A with six microRNAs (miR-17, miR-190b, miR-19a, miR-19b, miR-26b, and miR-375) provided high diagnostic accuracy in discriminating lung cancer patients from healthy individuals (AUC 0.873 and 0.868 for training and validation cohort, respectively). Moreover, plasma panel B with three microRNAs (miR-17, miR190b, and miR-375) demonstrated high diagnostic accuracy in discriminating SCLC from NSCLC (AUC 0.878 and 0.869 for training and validation cohort, respectively). Conclusions: We constructed and validated two plasma microRNA panels that have considerable clinical value in diagnosis of lung cancer, and could play an important role in determining optimal treatment strategies based on discrimination between SCLC and NSCLC.

1886 Spread through Air Spaces (STAS) Is an Independent Predictor of Recurrence in Lung Squamous Cell Carcinoma

Shaohua Lu, KS Tan, K Kadota, T Eguchi, S Bains, P Adusumilli, W Travis. MSKCC, New York, NY; Fudan University, Shanghai, China; Kagawa University, Kagawa, Miki-cho, Japan.

Background: STAS is recognized as a pattern of invasion in lung adenocarcinoma (AD) where it has been shown to be an independent predictor of recurrence in small lung AD. However, morphologic criteria for STAS and its prognostic impact have not been defined in squamous cell carcinoma. In this study, we investigated whether STAS occurs in resected lung squamous cell carcinoma, and whether it correlates with prognosis. Design: We reviewed a series of surgically resected, solitary lung squamous cell carcinomas ( $\mathrm{n}=445 ; \mathrm{M} / \mathrm{F}, 180 / 265 ; \mathrm{p}$-stage I/II/III, 249/131/65). STAS was defined as tumor nests spreading within air spaces in the lung parenchyma beyond the edge of the main tumor. We compared the cumulative incidence of recurrence (CIR) between groups using Gray's test, stratified by pathological stage. The effect of the STAS on recurrence, along with other factors, was evaluated using competing risk analyses. Results: STAS was observed in 132 cases (30\%), with limited (7.2\%) and extensive (22.5\%) morphologies, respectively. 5-year CIR was significantly higher in patients with STAS-positive (+) tumors compared to patients with STAS-negative (-) tumors $(39 \%$ versus $26 \% ; p=0.004)$. In the lobectomy group $(\mathrm{n}=376), 5$-year CIR was significantly higher in STAS+ than STAS- tumors ( $39 \%$ versus $27 \%, p=0.014$ ), whereas the difference was not significant in the limited resection group after stratification by pathological stage ( $44 \%$ versus $22 \%, p=0.242$ ). In multivariate analyses adjusting for smoking pack-years and $p$-stage, STAS was an independent predictor of recurrence (HR, $1.64 ; p=0.006$ ) but not of mortality $(p=0.3)$. STAS+ tumors showed significantly higher cumulative incidence of locoregional recurrence among stage I patients $(p=0.025)$, and higher cumulative incidence of distant recurrences among stage II/III patients $(p=0.019)$. Conclusions: STAS occurs in lung squamous cell carcinoma and is a strong predictor of recurrence, but not overall survival. Further evaluation and validation is needed to determine the prognostic significance and clinical implication of this finding.

1887 Non-Synonymous Mutation Burden in Lung Carcinoma Is Associated with Durable Clinical Response to Immune Checkpoint Blockade

Navin Mahadevan, Peter Hammerman, Mark Awad, Lynette M Sholl. Brigham and Women's Hospital, Boston, MA.

Background: Genes harboring non-synonymous somatic mutations (NSM) encode proteins containing potential immunogenic neoantigens. Recent evidence indicates that the efficacy and durability of responses to immune checkpoint inhibitors in carcinomas of different histologic origin, including lung, correlate with increased NSM burden, putative neoantigen number, alterations in DNA repair pathways, and in some tumor types, with PD-L1 protein expression. In this study, we retrospectively analyzed the relationship of lung carcinoma mutation burden and PD-L1 expression with clinical response in patients receiving immune checkpoint blockade.

Design: Somatic mutation data, including NSM, was gathered from targeted next generation sequencing (309 genes) of lung carcinomas from 21 patients treated with immune checkpoint inhibitors at our institution between 2012-2015, and was correlated with clinical outcome variables gathered from the EMR, including durable clinical benefit (DCB; $>6$ months partial or stable response by irRC) and progressionfree survival (PFS). PD-L1 immunohistochemistry (clone E1L3N, Cell Signaling Technology, Envision+ detection, Dako) was considered positive if $\geq 1 \%$ of tumor cells and/or tumor-infiltrating immune cells (IC) stained.

Results: The mean patient age was 61 years (range: $46-73$ years). The lung carcinomas included 16 adenocarcinomas, 2 squamous cell carcinomas, 2 adenosquamous carcinomas, and 1 small cell carcinoma. 13 and 2 patients received anti-PD1 antibodies, pembrolizumab or nivolumab, respectively. 6 patients received MPDL3280A, a monoclonal antibody against PD-L1. The mean PFS was 7.5 months. Across all lung carcinoma histotypes, patients who displayed a DCB had a significantly higher number of NSM (range 2-21) than patients who showed no durable benefit (NDB) [DCB: 13; NDB: $6, p=0.047]$. Patients who had greater than the median number of NSM (9) tended to have longer progression-free survival (10 vs. 4.6 months) than those with 9 or fewer NSM, though this trend did not reach statistical significance $(p=0.087)$. Expression of PD-L1 in either tumor cells or ICs was not associated with NSM burden $(p=0.85)$ nor with DCB $(p=0.5)$.

Conclusions: The NSM burden in lung carcinoma as detected by targeted next generation sequencing is associated with increased duration of response to immune checkpoint inhibitors. In this limited cohort, PD-L1 expression does not predict response to these therapies. We add to growing evidence that increased somatic mutations in carcinomas predict response to immune checkpoint blockade. 
1888 Programmed Death 1 (PD-1) and PD-L1 Expression in Lung Adenocarcinoma, with Lower Expression in In Situ and Minimally Invasive Tumors and Higher Expression in Larger and Invasive Tumors

Osamu Matsubara, Yasuto Jin, Shuta Yamauchi, Kazuteru Yamanaka, Yuichi Ishikawa, Eugene J Mark. The Cancer Insitute, Tokyo, Japan; Hiratsuka Kyosai Hospital, Hiratsuka, Japan; Massachusetts General Hospital and Harvard Medical School, Boston, MA.

Background: Immunopathological expression of programmed death 1 (PD-1) and its ligand PD-L1 play a key role in tumor immune escape and the formation of tumor microenvironment and is closely related to tumor progression. Blockading the PD-1/ PD-L1 pathway could alter the tumor microenvironment and enhance the endogenous antitumor immune responses. In lung cancer the precise conditions and diagnostic value of these immunological factors remains uncertain.

Design: Immunohistochemistry of PD-1 (NAT105, abcam), PD-L1 (EPR1161(2), abcam) and CD8 expression was performed in tumor infiltrating lymphocytes (TILs) and tumor cells in 113 cases with stage I-II lung adenocarcinoma resected by videoassisted thoracoscopic surgery or thoracotomy, including 19 cases with adenocarcinoma in situ (AIS), 3 cases with minimally invasive adenocarcinoma (MIA) and 91 cases with invasive adenocarcinoma. The membranous staining of any intensity in more than $10 \%$ of the tumor cells was considered positive. Histologic subtypes, tumor size and other clinicopathologic conditions were compared with the density of the expression. Results: PD-1 and PD-L1 expression in TILs and tumor cells was detected in 52.5\% and $58.7 \%$, respectively. The numbers of PD-1 positive TILs were significantly positively correlated with CD8 positive TILs. Cases displaying PD-L1 overexpression showed consistently dense CD8 positive TILs, even in subgroup analyses according to histological subtype, age, smoking status and the EGFR, MET and ALK status. The ratio of PD-1 positive/CD8 positive TILs was higher in smokers and in older patients. PD-L1 expression in tumor cells was significantly lower in AIS and MIA cases than in invasive adenocarcinoma cases. But, interestingly tumor cells showed PD-L1 expression in 5 of 19 cases (26.3\%) in AIS. It was significantly higher in tumors which were bigger than $3 \mathrm{~cm}$.

Conclusions: We identified PD-L1 overexpression in invasive adenocarcinoma which correlated positively with tumor size and negatively with in situ and minimally invasive adenocarcinoma and also correlated positively with denser PD-1/CD8 TILs. Activation of the PD-1/PD-L1 pathway influences the tumor microenvironment and progression of disease

1889 Does Strong and Diffuse PAX-8 Positivity Exclude Primary Lung Carcinoma? An Immunohistochemical Study of Whole-Tissue Sections from 337 Resected Primary Lung Carcinomas, and Review of 27 PAX-8Positive Carcinomas in Lung Specimens

Kelsey McHugh, Andrea V Arrossi, Carol Farver, Sanjay Mukhopadhyay. Cleveland Clinic, Cleveland, $\mathrm{OH}$.

Background: Previous studies, mainly tissue microarray-based, show that PAX-8 is rarely positive in primary lung cancer. The aim of this study was to determine whether strong and diffuse PAX-8 staining occurs in primary lung carcinoma. To approach the question from another perspective, we reviewed our experience with carcinomas in lung specimens that showed strong and diffuse PAX-8 positivity to determine if any of these subsequently proved to be primary lung tumors.

Design: Immunohistochemical staining for PAX-8 (Rabbit polyclonal, 10336-1-AP, Proteintech) was performed on whole-tissue sections from 337 resected primary lung carcinomas (177 adenocarcinomas, 52 carcinoid tumors, 34 large cell neuroendocrine carcinomas, 27 small cell carcinomas, 25 squamous cell carcinomas, 20 sarcomatoid carcinomas, 2 adenosquamous carcinomas). Additionally, we retrospectively reviewed all carcinomas in lung specimens that were read by pulmonary pathologists at our institution as strongly and diffusely positive for PAX-8 (2011-15). Twenty-seven carcinomas with strong and diffuse PAX-8 staining were identified in lung specimens. In each case, medical records were reviewed to determine if an extrapulmonary primary was documented.

Results: PAX-8 was positive in 5/337 (1.5\%) primary lung carcinomas. All 5 were large cell neuroendocrine carcinomas $(5 / 34,15 \%)$; staining ranged from weak to moderate, and was seen in $5-10 \%$ of tumor cells. All other primary lung carcinomas (332/337) were PAX-8-negative. Review of medical records in the 27 strongly and diffusely PAX8-positive carcinomas in lung specimens revealed a non-pulmonary primary in 26 , including kidney (17), endometrium (5), ovary (2), thyroid (1) and pelvis (1), confirmed histologically in 25 cases and radiologically in 1 . The site of origin was unclear in 1 patient with a history of thyroid carcinoma. There were no cases of primary lung carcinoma with strong and diffuse PAX-8 positivity.

Conclusions: This study, which represents the largest series of PAX-8-stained wholetissue sections of primary lung carcinoma to date, shows that strong and diffuse PAX-8 positivity does not occur in primary lung carcinoma. Focal weak to moderate PAX-8 positivity does occasionally occur in primary lung carcinoma, especially large cell neuroendocrine carcinoma. Strong and diffuse PAX-8 positivity in a carcinoma present in a lung specimen should prompt a search for an extra-pulmonary primary.

1890 Identification of Steroid Receptor Expression in Patients with Usual Interstitial Pneumonia

Mitra Mehrad, Humberto Trejo Bittar, Samuel A Yousem. University of Pittsburgh Medical Center, Pittsburgh, PA.

Background: Usual interstitial pneumonia (UIP) is characterized by progressive scarring of the lungs and is associated with high morbidity and mortality despite various therapeutic efforts. Recently, sex steroid receptors have been demonstrated to play an important role in pathological conditions in the lung such as cystic fibrosis, asthma and chronic obstructive pulmonary disease, however, the significance of these receptors is not well understood in patients with UIP.

Design: We retrospectively retrieved and reviewed 40 consecutive UIP cases. Diagnosis was made on tissue received from Video-Assisted Thoracoscopic Surgery wedge biopsies or native lung transplantation. Ten cases of unremarkable lung parenchyma from non-UIP patients were used as control cases. Immunohistochemical stains for estrogen receptor alpha (ER- $\alpha$ ), progesterone receptor (PR) and androgen receptor (AR) were performed in both groups. Within the UIP cases expression of these receptors was assessed in five areas of each of the following anatomic/pathologic compartments: alveolar and bronchiolar epithelium, blood vessels, mesenchyme including fibroblastic foci, old scar, and uninvolved parenchyma and compared to those in the control cases. Results: The majority $(75 \%)$ of patients were male. The mean and median age at diagnosis was 62.9 and 62, respectively. All UIP cases (100\%) were positive for PR in myofibroblasts in all five examined scarred areas and $34(85 \%)$ showed positivity in smooth muscle cells in at least one scarred region. PR was positive in myocytes of the large-sized vessels within the fibrotic areas in 31 cases (77.5\%). In addition, only 11 cases $(27.5 \%)$ showed PR expression in the uninvolved lung. PR was negative in the fibroblastic foci, endothelium, and alveolar and bronchial epithelium. Among the control cases, PR was expressed in a minority $(30 \%)$, seen within the fibroblasts of the alveolar septa. All cases and controls were uniformly negative for AR and ER- $\alpha$, with adequate controls.

Conclusions: To our knowledge this is the first study to evaluate the role of steroid receptors in UIP. The increased expression of PR mostly within the established fibrotic areas, indicate that progesterone may have profibrotic effects through its receptors in UIP patients, regardless of gender. Our findings suggest hormonal therapy by targeting PR may be of potential benefit in patients with this devastating disease.

\section{KRAS Mutations Are Predictive of Outcome in Patients with} Pulmonary Sarcomatoid Carcinoma

Mitra Mehrad, Pimpin Incharoen, Marina Nikiforova, Humberto Trejo Bittar, Sanja Dacic. University of Pittsburgh Medical Center, Pittsburgh, PA; Mahidol University, Bangkok, Thailand.

Background: Pulmonary Sarcomatoid Carcinoma (SC) includes five different subtypes of a poorly differentiated non-small cell lung carcinoma with an aggressive behavior. The aim of this study was to evaluate the prognostic significance of clinicopathologic and molecular characteristics of SC.

Design: Forty-three cases of surgically treated SC, including pleomorphic carcinoma $(42 / 43,97.7 \%)$ and carcinosarcoma $(1 / 43,2.3 \%)$ were selected for the study. Adenocarcinoma was found in $29(67.4 \%)$, squamous cell carcinoma (SCC) in 7 $(16.2 \%)$, adenosquamous carcinoma in $5(11.6 \%)$ and large cell neuroendocrine carcinoma in $2(4.6 \%)$ cases. Clinical information including gender, age, tumor stage and smoking status were obtained from patient's electronic medical records. Follow-up data with regard to first recurrence and survival were collected through the institutional Network Cancer Registry. Sangers sequencing for $E G F R, K R A S, B R A F$ and PIK3CA and FISH for $A L K, R O S 1, R E T$ and $M E T$ were performed on $27 \mathrm{SC}$ with adenocarcinoma component. FISH for FGFRI amplification and PIK3CA mutation analysis were performed on SC with SCC component.

Results: The majority of patients were male $(26 / 43,60.4 \%)$ and smoker $(41 / 43,95.3 \%)$ with median age of 66 years at diagnosis. The tumors ranged in size from 1.3 to $10 \mathrm{~cm}$ (median $4.5 \mathrm{~cm}$ ). The majority of cases showed angiolymphatic invasion $(31 / 43,72.1 \%)$ and visceral pleural invasion was present in 20 cases (46.5\%). The most common genetic alteration in SC with adenocarcinoma component was codon 12 KRAS mutation (16/27, $59.2 \%)$. EGFR mutations were identified in $2(2 / 27,7.4 \%)$ and $M E T$ amplification in one $(3.7 \%)$ case. No FGFR1 amplification or PIK3CA mutation were identified. Mean follow-up duration was 29 months. Overall, 16 of 25 (64\%) patients died within 1 to 34 months (mean 11.2) after diagnosis, and 18 were alive as of 5 to 118 months (mean 39) after diagnosis. Five-year overall survival (OS) was $12.5 \%$ for the whole population. At univariate analysis, KRAS ( $\mathrm{p}=0.017$ ) mutations were associated with significantly decreased survival (mean survival of 24.9 versus 32.6 months in wild-type $K R A S$ ). There was no sufficient data to reliably assess prognostic significance of EGFR mutations and $M E T$ amplification. No correlation was found between clinicopathologic characteristics, patient demographics and outcome.

Conclusions: Our data suggests that KRAS codon 12 mutations are the most common genetic alteration in $\mathrm{SC}$ and the most important predictor of aggressive behavior in SC with adenocarcinoma component.

\section{Next Generation Sequencing Approach to Non-Small Cell Lung} Carcinoma Testing Yields More Actionable Results

Mitra Mehrad, Marina Nikiforova, Humberto Trejo Bittar, Sanja Dacic. University of Pittsburgh Medical Center, Pittsburgh, PA.

Background: The current guidelines recommend testing for $E G F R$ mutations and $A L K$ rearrangements in all patients with advanced-stage lung adenocarcinoma. Additional gene targets have been proposed, and it is unclear if the patients would benefit from broader gene panel testing. In our institution, metastatic adenocarcinomas are subjected to a reflex testing for 8-gene panel composed of Sangers sequencing (SS) for $E G F R$, $K R A S, P I K 3 C A$ and BRAF and FISH for ALK, ROS1, MET and RET. At the oncologist request, a subset of wild-type tumors can be subjected to a 50 -gene mutation panel by Ion Torrent AmpliSeq. The aim of this study was to determine whether a large gene panel testing by NGS would improve the detection of actionable genetic abnormalities. Design: A total of 120 NCLCs were subjected to NGS, of which 35 cases were wild-type by SS and were subjected to sequencing by Ion Torrent. Cases included: $26(74.3 \%)$ adenocarcinoma, $5(14.2 \%)$ large cell neuroendocrine carcinoma and $4(11.5 \%)$ poorly differentiated non-small cell carcinoma. 
Results: Among the 35 cases, NGS detected 9 actionable variants (9/35, 25.7\%), occurring in the following genes: ERBB2 (3, 8.5\%), FBXW7 (2, 5.7\%), BRAF G469A $(1,2.8 \%), \operatorname{RET}(1,2.8 \%), \operatorname{MET}(1,2.8 \%)$ and PTEN (1, 2.8\%). In addition, NGS detected codon 12 KRAS mutation in one case that was missed by Sangers sequencing. All actionable mutations were mutually exclusive. Four of the mutations [PTEN (1), $E R B B 2(2), F B X W 7(1)]$ were found in adenocarcinomas, $3[M E T(1), R E T(1), F B X W 7$ (1)] in LCNEC and $2[B R A F(1), E R B B 2(1)]$ in poorly differentiated non-small cell carcinomas. Overall, 70 variants of uncertain clinical significance were found, most common being TP53 (30, 42.8\%).

Conclusions: Our data shows that testing for a larger gene panel by NGS would result in identification of a significantly high number of actionable gene alterations, increasing the therapeutic options in patients with NSCLCs. Our data argues against multistep approach to molecular testing and favors reflex NGS approach to NSCLC molecular testing.

1893 Pleuropulmonary Solitary Fibrous Tumors- A Clinicopathologic Study of 84 Cases with Comparison of Proposed Risk Stratification Systems

Wadad Mneimneh, Sarah M Jenkins, Marie Christine Aubry, Jennifer M Boland. Mayo Clinic, Rochester, MN

Background: Solitary fibrous tumors (SFTs) are mesenchymal neoplasms characterized by NAB2-STAT6 fusion. Most follow a benign course, but a subset will recur or metastasize. Various risk stratification schemes have been proposed for SFT, but none have been universally accepted or endorsed by the WHO classification of lung and pleural tumors. The purpose of this study is to perform a clinicopathologic analysis of pleuropulmonary (PP) SFTs and compare performance of several risk prediction models. Design: Histologic sections were examined from 84 excised PP SFTs (1997-2013). Immunohistochemistry for CD34 and STAT6 was performed. Follow-up data was collected from clinical records. Kaplan-Meier analysis was used to estimate overall survival (OS). Tumors were risk stratified using the following proposed schemes:

-Demicco et al: Includes age, size and mitoses, and stratifies patients to low, moderate, or high risk.

-DePerrot staging system: Includes pedunculation and "histologic features of malignancy" (cellularity, pleomorphism, mitoses, necrosis, stromal/vascular invasion), dividing patients into 5 stages $(0-4)$.

-Tapias et al: Includes pedunculation, site (parietal or visceral), size, cellularity, necrosis, hemorrhage, and mitoses, dividing patients into low and high risk.

Results: Patients included 51 females and 33 males with a median age of 64 years (26-87). Mean follow up was 5.3 years (0-21). Immunostains were performed in 76 cases: all were STAT6 positive, while $74(93 \%)$ were CD34 positive. Adverse outcomes occurred in 4 patients (5\%, 3 recurrences, 2 metastases), with 2 deaths from disease and 11 deaths from any cause. The 4 patients with progressive disease were all high risk by Tapias method; and 3 were high and 1 moderate by Demicco system; using DePerrot staging, 1 was stage 2, 2 stage 3 , and 1 stage 4 . Significant adverse predictors of OS included male gender, extrapulmonary location, severe atypia, necrosis, age, size, and mitoses; pedunculation was not significant. The ability of the DePerrot system to predict OS did not reach significance $(\mathrm{p}=0.08)$, while both Tapias and Demicco models performed well in predicting $\mathrm{OS}$ ( $\mathrm{p}=0.003$ and 0.006 respectively).

Conclusions: The scoring systems proposed by Tapias and Demicco were both predictive of OS, while DePerrot stage did not significantly correlate with outcome. The Demicco system has the advantage of simplicity, and thus may be the best system to apply in general practice.

1894 Lessons Learned from Next Generation Sequencing in Use of Immunohistochemistry (IHC) for EGFR L858R and ALK in Lung Cancer Predictive Testing

Joseph Montecalvo, C Leduc, N Rekhtman, A Moreira, M Arcila, M Berger, C Rudin, $V$ Rusch, D Jones, $W$ Travis. MSKCC, New York, NY; Mayo Clinic, Rochester, MN. Background: Introduction of next generation sequencing (NGS) has been a great advance for molecular testing of lung cancers, however, since patients with advanced disease often need rapid results to initiate therapy it has also presented some challenges. Design: We analyzed 1 year of biopsies managed by the thoracic pathology service beginning September 2014 using NGS using the Illumina HiSeq custom capture based platform analyzing mutations and copy number of 341 genes. Due to the 4 week delay for NGS results, to obtain more rapid results for key actionable mutations we test by IHC using mutation specific antibodies for ALK (D5F3, Ventana) and EGFR L858R (43B2, Cell Signaling) and Sanger sequencing (SS) for EGFR exon 19, EGFR exon 20 and ERBB2/HER2 exon 20 deletions. We focused on discrepant results in the 288 cases studied by NGS versus IHC and ALK FISH.

Results: In the 288 cases examined for NGS, 234 were AD, 31 squamous, 5 small cell, 5 LCNEC including one combined with $\mathrm{AD}, 10$ nonsmall cell, 2 pleomorphic and 1 adenosquamous carcinoma.

EGFR mutations were found in L858R (NGS) and Exon 19 deletions (SS) in 27 (9\%) and $39(14 \%)$ of cases (all with $\mathrm{AD}$ ) respectively. Other therapeutically relevant mutations found included KRAS:89 (31\%). BRAF: 11 (4\%), ERBB2/HER2:3 (1\%), RB: $2(0.7 \%)$, and $A K T: 1(0.3 \%)$. ROSI fusion was found in $1(0.3 \%)$.

Of 618 cases stained for EGFR L858R, 45 were positive (7.3\%, all ADC) and 9 were equivocal (1.5\%). In cases with NGS, in all 25 EGFR L 858R mutant cases IHC showed either positive $(21,88 \%)$ or equivocal $(3,12 \%)$ staining. However, IHC was positive in 3 of $64(5 \%)$ cases without mutation. Of false-positive cases two showed ERBB2 amplification (fold change of 8 and 19) and a third showed an $E G F R$ exon 19 deletion. Positive HER2 IHC was seen in one of the ERBB2 amplified cases.

Of 630 cases stained for $\mathrm{ALK}, 18$ were positive (all AD, 2.9\%) and 5 were equivocal. All 8 cases positive for ALK IHC and one of 4 equivocal cases showed ALK rearrangement by NGS and/or FISH. In one case confirmed by NGS, ALK IHC was negative.
Conclusions: Our data suggests false positive IHC for EGFR L858R can rarely occur in the setting of ERBB2/HER2 amplification and EGFR Exon 19 deletion. While IHC for $E G F R$ mutation is used for therapeutic decisions, it remains useful to perform molecular testing using NGS. Further study is needed to see if IHC for HER2 may be a useful marker for ERRB2/HER2 amplification as a cause of false positive EGFR L858R staining.

1895 Venous Infarcts and Pathologic Changes Mimicking Pulmonary Venoocclusive Disease Caused by Pulmonary Vein Obstruction outside the Lungs

Sanjay Mukhopadhyay, Andrea V Arrossi, Carol Farver. Cleveland Clinic, Cleveland, $\mathrm{OH}$.

Background: Pulmonary venoocclusive disease (PVOD) is a rare vasculopathy caused by occlusion of intrapulmonary venules. We present a series of cases in which pathologic changes similar to PVOD were caused by pulmonary vein obstruction outside the lungs. Design: We identified 4 surgical lung biopsies in which venous infarcts and other PVOD-like changes were identified in patients with clinical evidence of pulmonary vein obstruction outside the lung. Clinical, radiologic and pathologic findings in these cases were reviewed.

Results: All 4 patients were young men (25-39 years). Presenting symptoms included cough (3/4), chest pain (3/4), dyspnea (2/4) and hemoptysis (2/4). Chest CT findings in the lung parenchyma included unilateral nodular densities or opacities in all 4 cases, which progressed to consolidation in 1 and were associated with interstitial changes in 1. The clinico-radiologic differential diagnosis included malignancy, infection, septic emboli and granulomatosis with polyangiitis. Infarction was suspected clinically in only 1 case. Obstruction of the pulmonary vein was noted radiologically in all 4 cases. The cause of obstruction was fibrosing mediastinitis in 2 cases (with rare Histoplasma yeasts seen on biopsy in 1, typical radiologic features in the other), bronchogenic cyst in 1 (confirmed pathologically) and pulmonary vein stenosis following radiofrequency ablation for atrial fibrillation in 1 (CT angiogram performed after lung biopsy showed findings suspicious for pulmonary vein obstruction, as well as pulmonary emboli). Pathologic findings in all 4 cases included hemorrhagic subpleural or paraseptal ("venous") infarcts surrounded by a rim of fibroblasts mimicking organizing pneumonia, mild interstitial thickening by congested capillaries, and variable numbers of hemosiderin-laden macrophages within airspaces. The infarcts contained hematoidin in 3 cases. Infarction of interlobular septa was noted in 2 . The pleura was thickened by prominent granulation tissue in 3 cases and contained congested vessels in the fourth. All 4 cases showed pulmonary vascular changes secondary to venous obstruction, including congestion and dilatation of veins and capillaries, and intimal fibrosis and medial hypertrophy of arteries. These changes were severe enough to merit consideration of a vascular malformation in 1 case and pulmonary hypertension in 2 cases.

Conclusions: Obstruction of pulmonary veins outside the lungs can cause venous infarcts, interstitial changes and secondary vascular abnormalities that can mimic PVOD and other vasculopathies.

1896 Aggressive B-cell Lymphomas of the Respiratory System: Histologic, Immunohistochemical and Genetic Evaluation of a Large Series Yalda B Naeini, Annie Wu, Dennis O'Malley. UCLA, Los Angeles, CA; Clarient Pathology Services, Aliso Viejo, CA.

Background: Diffuse large B-cell lymphoma and related entities are the commonest type of non-Hodgkin lymphomas. However primary involvement of respiratory system by aggressive $\mathrm{B}-$ cell lymphoma (ABL) is extremely uncommon, diagnostically challenging and poorly studied.

Design: 760 consult cases that were diagnosed as ABL including Burkitt lymphoma (BL), B-cell lymphoma unclassifiable, including double hit lymphomas (DHL), and diffuse large B-cell lymphoma (DLBCL) were evaluated using an extensive panel of immunohistochemical stains (CD20, CD3, CD5, CD10, Cyclin D1, BCL6, BCL2, EBER, Ki-67, CD30) and a panel of FISH studies (including MYC, IgH/BCL2 and BCL6). Forty-four ( $5 \%$ ) cases were identified in the respiratory tract; $43 \%$ were located in nasopharynx.

Results: The majority of cases were DLBCL $(37 / 44 ; 84 \%)$, with two cases of BL (4\%), three lymphomatoid granulomatosis $(6 \%)$, one DHL ( $2 \%$ ) and one plasmablastic lymphoma (2\%) identified. MYC translocation was only identified in BL and DHL. Compared to a large group of lymphoma in other organs, respiratory tract lymphomas were more often positive for EBV ( $25 \%$ vs. $8 \%)$ and were of non-germinal center origin $(\sim 67 \%)$ by both Hans and tally classifiers (compared to $60 \%$ germinal center origin in other organs)

Conclusions: Our series shows that aggressive lymphomas in respiratory tract show heterogeneity of immunophenotype and genetics. Also the cell of origin in respiratory tract lymphoma differs from the large group of lymphoma in other organs ( $33 \% \mathrm{GC}$ vs $60 \% \mathrm{GC})$.

1897 Diagnostic Utility of Orthopedia Homeobox (OTP) in Pulmonary Carcinoid Tumor

Daisuke Nonaka, George Papaxoinis, Was Mansoor. The Christie Hospital, Manchester, United Kingdom; The University of Manchester, Manchester, United Kingdom. Background: Recently OTP (Orthopedia Homeobox) has been described as a prognostic marker for pulmonary carcinoid tumors, however, little is known about the function and distribution pattern of this transcription factor in normal organs/tissues and tumors, herein, OTP immunohistochemistry expression was investigated on a number of tumors, with special interest in pulmonary and non-pulmonary neuroendocrine tumors and high grade neuroendocrine carcinomas. 
Design: OTP immunohistochemistry was performed on a total of 164 pulmonary carcinoid tumors including 157 primary (140 resections: 121 typical 19 atypical; 17 biopsies) and 7 metastatic tumors, 26 cases of pulmonary neuroendocrine hyperplasia, 104 pulmonary high grade neuroendocrine carcinomas (11 large cell neuroendocrine, 93 small cell), 102 non-pulmonary neuroendocrine tumors ( $52 \mathrm{G} 1 / \mathrm{G} 2$ neuroendocrine tumors, 12 small cell carcinomas, 30 Merkel cell carcinomas, 8 large cell neuroendocrine carcinomas), 150 endocrine tumors (73 thyroid, 7 parathyroid, 27 adrenocortical, 20 pheochromocytomas, 23 paragangliomas), 279 adenocarcinomas and 88 squamous cell carcinomas of various organs, including those of lung, other types of carcinomas, and melanomas. Additionally, normal tissues from various organs except for CNS were studied. The extent of nuclear staining was graded as follows: $1+, 1-25 \% ; 2+, 25-50 \%$; $3+, 50-75 \% ; 4+, \geq 75 \%$.

Results: OTP expression was seen in $81 \%$ of lung carcinoid tumors ( $88 \%$ in typical, $49 \%$ in atypical), and $4+$ staining was seen in $75 \%$ of the tumors. Among other tumors, 4 small cell carcinomas showed focal expression $(1+$ in 2 pulmonary, $1+$ and $2+$ in 2 bladder tumors), but all other tumors were completely negative. Overall the sensitivity and specificity of OTP was $80.9 \%$ and $99.7 \%$, respectively. Notably, all TTF1 positive tumors were positive for OTP with $3+$ or $4+$ reaction, but none of OTP negative tumors were positive for TTF1. OTP expression was not seen in any normal tissues/organs. OTP was also negative in neuroendocrine cells of the normal bronchus/bronchiole, however, OTP was strongly expressed in neuroendocrine hyperplasia, including reactive and preneoplastic hyperplasia (DIPNECH).

Conclusions: OTP may serve as a useful diagnostic marker for lung carcinoid tumors, particularly in the setting where the differential diagnosis is lung carcinoid vs. small cell carcinoma, or where lung origin needs to be confirmed in metastatic well differentiated neuroendocrine tumors.

\section{Absence of Nuclear BAP1 Protein Expression Is Highly Sensitive for Loss-of-Function BAP1 Genomic Alterations in Malignant} Mesothelioma

Prodipto Pal, Fei Dong, Marina Vivero. Brigham and Women's Hospital, Boston, MA. Background: Somatic alterations of the BAPl tumor suppressor gene have been reported as a defining event in up to $60 \%$ of pleural diffuse malignant mesotheliomas (PDMM). Absence of nuclear BAP1 staining by immunohistochemistry (IHC) has likewise been reported in up to $60 \%$ of PDMM, with good correlation to BAPl genetic alterations, and has therefore been proposed as a diagnostic marker. Systematic correlation between BAP1 IHC and genetic alterations to include sequence alterations, copy number variation (CNV), and rearrangements, however, is lacking. The aim of this study is to correlate BAP1 IHC with gene-level changes in PDMM to support its use as a clinical tool. Design: Included were 78 PDMM and one peritoneal mesothelioma with available next generation sequencing (NGS) data collected between 2013-2015. Targeted hybrid capture NGS for 309 cancer-related genes including $B A P 1$ was performed for detection of single nucleotide variants (SNVs), indels, CNVs, and rearrangements. BAP1 (Santa Cruz C4 antibody; dilution 1:50) IHC was performed on 4 micron formalin-fixed paraffin embedded tissue sections following pressure cooker antigen retrieval, and scored as positive or negative.

Results: The study included 47 (59\%) epithelioid, 6 (8\%) sarcomatoid and $26(33 \%)$ biphasic PDMM. Overall, $54(68 \%)$ tumors had BAP1 alterations, including $2(2.5 \%)$ homozygous deletions (2DEL), $1(1 \%) 142$ base pair deletion, $2(2.5 \%)$ rearrangements, $6(8 \%)$ loss-of-function SNV or indels, $18(23 \%)$ biallelic alterations, $8(10 \%)$ SNV of unknown significance (VUS), and 9 (11\%) heterozygous deletions (1DEL). Of these 54 cases, $48(89 \%)$ showed loss of nuclear BAP1 IHC expression. 6 cases with genetic alterations demonstrated weak/heterogeneous BAP1 nuclear expression, of which 3 harbored 1DEL and 3 harbored VUS. No WT cases had BAP1 loss. Excluding 1DEL and VUS as alterations of unknown functional significance, loss of nuclear BAP1 expression yielded a sensitivity of $100 \%$, specificity of $72 \%$ positive predictive value of $75 \%$ and negative predictive value of $100 \%$ for deleterious $B A P 1$ alterations.

Conclusions: Complete loss of BAP1 IHC expression is highly sensitive for loss-offunction $B A P 1$ genomic alterations in PDMM . A minority of cases showed BAP1 loss in the absence of confirmed functional alterations, which may reflect limitations of IHC, limitations of NGS, or additional mechanisms of BAP1 deregulation. In summary, our results support the use of BAP1 IHC as a diagnostic tool and potential screening assay for significant $B A P 1$ genomic alterations.

\section{Clinico-Pathologic Characteristics of Primary Lung Carcinoma} with Hepatoid Differentiation

Prodipto Pal, Lucian Chirieac. Brigham and Women's Hospital, Boston, MA.

Background: Primary lung carcinoma with hepatoid morphology and differentiation is an exceedingly rare group of aggressive tumors. Distinction from primary hepatocellular carcinomas with hepatoid morphology is critical as there is direct impact in clinical and therapeutic decisions. In this study, we describe the clinico-pathologic characteristics and outcome in a series of 9 patients with primary lung carcinoma with hepatoid differentiation and characterize the histopathological parameters that may aid in diagnosis of these tumors.

Design: A search of the surgical pathology database from Brigham and Women's Hospital between January 2005 and August 2015 identified 7 women (median age, 58 yrs, range $45-78$ years) and 2 men (median age, 44.5 years, range $42-47$ years) with a diagnosis of lung carcinoma with hepatoid differentiation. Clinical presentation, staging data and survival were obtained from review of clinical charts. All patients included in this study had no evidence of primary liver tumors. Immunohistochemical studies were available for TTF-1, CK7, hepatocytic markers (Hep Par-1, arginase-1, glypican 3 ) and polyclonal CEA (pCEA) in the majority of cases.

Results: Eight of our patients had documented smoking history (range, $<1$ pack year to 100 pack years). All the tumors had hepatoid cytomorphology with predominantly solid architectural pattern and variable amounts of necrosis. Tumor cells showed distinct cell membranes, abundant cytoplasm, moderate to severe pleomorphism, vesicular nuclear chromatin and prominent nucleoli. Eight cases ( $89 \%$ ) had cytoplasmic granular staining for TTF-1, CK 7 (100\% of evaluated cases), pCEA in 44\% and expression for hepatocytic markers (Hep Par- 1 in $89 \%$, arginase- 1 in $22 \%$ and glypican-3 in $11 \%$, respectively). Average follow up time was 6 months (median, 17 months; range 0-21 months). Majority ( $89 \%$ ) of the patients had advanced clinical stage (stage III/IV) at presentation. Conclusions: Lung carcinomas with hepatoid differentiation are exceedingly rare and have unique clinicopathologic characteristics that are distinctive from the conventional primary lung adenocarcinomas. Tumors consistently have cytoplasmic TTF-1 (and lack of nuclear TTF-1), CK7 and Hep Par-1 expression. In our series the majority of the patients with lung carcinomas with hepatoid differentiation are younger, present in advanced clinical stages and have a worse prognosis.

\section{ROS1 Immunohistochemistry as a Screen for ROS1 Rearrangements} in Lung Adenocarcinoma: Application in Clinical Practice

Prodipto Pal, Peter Hammerman, David J Kwiatkowski, Bruce E Johnson, Lynette M Sholl. Brigham and Women's Hospital, Boston, MA; Dana Farber Cancer Institute, Boston, MA.

Background: $R O S 1$ gene rearrangements occur in 1-2\% of lung adenocarcinomas and predict response to crizotinib therapy. The optimal approach to ROS1 translocation detection has not yet been established, however many labs depend on fluorescence in situ hybridization (FISH). FISH is costly and time consuming; immunohistochemistry (IHC) for ROS1 protein is a promising screening tool, although there is limited published data on its use in routine clinical practice. In this study we examine the performance of ROS1 IHC in practice including its correlation with FISH and next generation sequencing (NGS).

Design: Upon clinical request, ROS1 IHC was carried out using clone D4D6 (Cell Signaling Technology) on 4 micron sections and reported as negative, equivocal (weak/ focal staining) or positive (moderate to strong, diffuse). All IHC positive and equivocal cases were analyzed by FISH; specimens from patients enrolled in the Lung Cancer Mutation Consortium study underwent FISH testing regardless of IHC result. FISH testing was carried out using commercially available ROS1 breakapart probes (Kreatech) using published procedures. Targeted hybrid capture next generation sequencing (NGS) for 309 cancer-related genes was performed for detection of mutations, indels, copy number variants $(\mathrm{CNV})$, and rearrangements in a subset of cases.

Results: From July 2013 to September 2015, ROS1 IHC was performed on 943 lung tumors. Positive ROS1 protein expression was detected in 19 cases $(2 \%)$. Of these, FISH confirmed a rearrangement in 8 , was negative in 7 , and gave no result in 4 . Equivocal ROS1 staining was reported in 40 cases (4\%); FISH was successful in 27 equivocal cases and all were negative for rearrangement. Of 69 cases that were IHC negative but for which FISH was requested, ROS1 FISH was successful in 58 cases and all were negative for rearrangement. NGS results were obtained in cases with equivocal $(n=2)$ and positive $(\mathrm{n}=2)$ ROS1 IHC staining that failed FISH; both positive cases contained a CD74-ROS1 fusion. No ROS1 fusions were detected by NGS in the equivocal cases. Conclusions: ROS1 protein detected as moderate to strong and diffuse staining by IHC is $100 \%$ sensitive and $93 \%$ specific for a rearrangement by FISH or NGS. Tumors with negative or equivocal ROS1 expression are consistently negative for ROS1 rearrangement. ROS1 IHC is a reliable screening tool in clinical practice; reflexive confirmation using FISH or molecular techniques should be restricted to tumors with moderate to strong and diffuse expression.

\section{Molecular Characterization of Invasive Mucinous Adenocarcinomas of the Lung}

Kyung Park, Shivakumar Subramaniyam, Jose Jessurun, Helen Fernandes, Navneet Narula. NYPH-WCMC, NYC, NY.

Background: Invasive mucinous adenocarcinomas of the lung (IMA) are clinically and genetically distinct from non-mucinous subtypes. Recent studies suggest that IMA share some molecular signatures with colorectal and pancreatobiliary carcinomas. In this study, we investigated the mismatch repair profiles and microsatellite instability (MSI) status of these tumors. In addition, we attempted to correlate the presence of KRAS mutations with the tumor type.

Design: The cohort consisted of 60 lung resection specimens collected from 2001 to 2013 at New York Presbyterian Hospital-Cornell Campus. Tumors were classified based on the International Association for the Study of Lung Cancer/American Thoracic Society/European Respiratory Society (IASLC/ATS/ERS) classification. A tissue microarray (TMA) composed of two representative cores of each tumor was constructed from formalin-fixed paraffin-embedded (FFPE) tissue blocks. Immunohistochemistry (IHC) using antibodies against MLH1, PMS2, MSH2, and MSH6 was performed. Thirteen cases with equivocal or negative immunostaining were subjected to PCRbased MSI testing. KRAS mutation status of the tumor tissue was determined by Sanger sequencing following DNA extraction from FFPE tissue.

Results: Sixty patients with IMA including $22(37 \%)$ males and $38(63 \%)$ females with a median age at diagnosis of 71.5 years were analyzed. The majority of patients had lower T stage: $30(50 \%)$ with T1; $15(25 \%)$ with T2; 11(18\%) with T3; $4(7 \%)$ with T4. Six patients had lymph node metastases and one patient had M1 disease at the time of resection. $38 / 57(67 \%)$ had a history of smoking and $19(33 \%)$ were non-smokers. The entire cohort was microsatellite stable with functional mismatch repair genes. 17 of the $26(65 \%)$ cases interrogated, harbored the following variants in the KRAS gene: 4 patients with G12C, 8 patients with G12V, 4 patients with G12D, and 1 patient with both G12V and G12D. $75 \%$ of the KRAS variants were $\mathrm{G} \rightarrow \mathrm{T}$ transversions and $25 \%$ were $\mathrm{G} \rightarrow \mathrm{A}$ transition mutations. Patients with $K R A S$ variants had higher tumor stage (T3 or T4) compared to those without mutations; $29 \%$ and $0 \%$, respectively. There was one $B R A F$, one $E G F R$ and $2 A L K$ translocations that were detected in the cohort. 
Conclusions: This study illustrates that IMA are microsatellite stable with a high prevalence $(65 \%)$ of $K R A S$ mutations. Transversions are more frequent than transition mutations. Additional molecular analyses would help in the further characterization of this subset of tumors.

\section{Neoadjuvant Treatment Response Predicts Survival in Non-Small} Cell Lung Cancer Resections

Adina Paulk, Alexander A Berrebi, Allen P Burke. University of Maryland Medical Center, Baltimore, MD.

Background: Neoadjuvant chemotherapy or radiation is often used in Stage III nonsmall cell lung carcinoma prior to surgical resection. Few studies have investigated the predictive potential of percent treatment response in lung cancer resections; no study has investigated the effect of neoadjuvant therapy on metastatic disease in lymph nodes. Design: We retrospectively reviewed 61 resections for non-small cell lung cancer following neoadjuvant therapy performed between 2003-2012. Specimens were evaluated for percentage of residual tumor. Electronic medical records were utilized to track length of survival from time of resection, and disease specific survival. Kaplanmeier survival curves were generated for tumors with $100 \%$ response and $<100 \%$ treatment response.

Results: The M:F ratio was 0.9 with mean age of 58 . Percentage of treatment response ranged from $20-100 \% .40 / 61(63 \%)$ of specimens showed $100 \%$ treatment response in the primary tumor. Of these, 14 patients were deceased $(35 \%)$, with 3 dead of progressive disease. Of the $21(34 \%)$ patients with $<100 \%$ residual tumor, $13(42 \%)$ were deceased with 6 dead of progressive disease. Kaplan-meier survival analysis showed superior survival for patients with $100 \%$ treatment response compared to all patients with $<100 \%$ response ( $\mathrm{p}=.04$ ) (see figure 1 ). 59 patients underwent lymph node dissection. Residual metastatic tumor was present in lymph nodes in 5 patients $(8 \%)$; all but 1 of these patients also had incomplete treatment response in the primary tumor.

\section{Survival Plot}

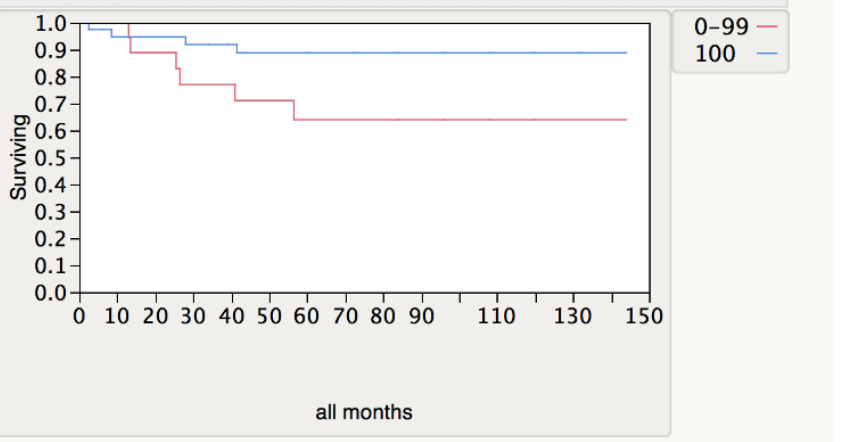

Conclusions: Complete treatment response is associated with a superior prognosis. Residual lymph node disease was uncommon and seen most often in cases where there was incomplete treatment response in the tumor.

1903 Dissecting Intra-Tumor Heterogeneity of Lung Adenocarcinoma Reveals Dominant, Branching and Private Gene Mutations within Individual Tumor Nodules

Giuseppe Pelosi, Alessio Pellegrinelli, Alessandra Fabbri, Adele Busico, Giulio Settanni, Barbara Valeri, Angelica Sonzogni, Federica Perrone, Elena Tamborini, Ugo Pastorino. Fondazione IRCCS Istituto Nazionale Tumori and Università degli Studi, Milan, Italy; Fondazione IRCCS Istituto Nazionale Tumori, Milan, Italy.

Background: While lung adenocarcinoma (ADC) is morphologically heterogeneous, little is known about intra-tumor gene mutation heterogeneity (ITH).

Design: Twenty ADC nodules mutated for EGFR ( 5 cases) or $K R A S$ ( 5 cases), with $A L K$ translocation ( 5 cases) or wild type (WT) for these alterations ( 5 cases) were subjected to unsupervised targeted next generation sequencing in diversely patterned tumor regions by using the 50-gene Ion AmpliSeq Cancer Hotspot Panel v. 2 running on the Ion-Torrent ${ }^{\mathrm{TM}}$ Personal Genome Machine platform (Life Technologies-Thermo Fisher Scientific, USA). Two or more gene mutations per single tumor fulfilled criteria for ITH. Results: A total of 84 tumor regions with diverse architecture accounted for 71 gene mutations and $34 \mathrm{WT}$ profiles. ITH was observed in 3 EGFR, 3 KRAS and 3 ALK tumors, with the finding of 5,6 and 9 additional mutations, respectively. EGFR mutations were found in 21/22 (95\%) and KRAS mutations in 18/22 (82\%) tumor regions, suggesting earlier appearance and driver role (dominant or trunk mutations), but with a temporally later emergence of $K R A S$ compared to $E G F R$ mutations. There were also branching mutations (EZH2, PIK3CA and TP53) occurring in two or more regions, which supported some driver role for them in the development of individual tumor nodules. In turn, ABL1, ALK, BRAF, HER2, KDR, LKB1, PTEN, MET, SMAD4, SMARCB1 and SRC were private/passenger/hitchhiker mutations confined to single tumor spots of individual lesions, which were likely to be involved in the growth of single tumor sub-clones (and then temporally delayed) but potentially responsible for unexpected secondary resistance upon therapy. EGFR was mutated in low-grade (lepidic, papillary and acinar) tumor regions, while $K R A S$ or $A L K$ translocation-associated mutations occurred in high-grade (solid, acinar-cribriform, micropapillary and high-grade fetal) tumor spots $(\mathrm{p}<0.0001)$. Branching mutation patients ran a worse clinical course $(\mathrm{p}=0.040)$.

Conclusions: ITH accounted for a spatial/temporal hierarchy of dominant, branching and private mutations, suggesting diverse clonal evolution with potential implications for patient prognosis or secondary resistance development upon therapy.
1904 Proliferation Activity Evaluation by Ki-67 Labeling Index in Paired Biopsy Samples and Surgical Specimens of Pulmonary Neuroendocrine Tumors: A Methodological Approach for Clinical Purposes

Giuseppe Pelosi, Mara Cossa, Alessandra Fabbri, Barbara Valeri, Angelica Sonzogni, Ugo Pastorino. Fondazione IRCCS Istituto Nazionale dei Tumori, Milan, MI, Italy. Background: Although classification of lung neuroendocrine tumors (NET) is based on the histologic assessment of surgical specimens (SS), little is known about the use of proliferation-related indexes in biopsy samples (BS) of these tumors for clinical purposes

Design: Twenty-one lung NET outlined according to 2015 WHO classification were assessed for Ki-67 antigen by immunohistochemistry, mitoses and necrosis in paired BS and corresponding SS. In each tumor case, Ki-67 was counted manually as the percentage of positive tumor cells by scanning hot spot areas (HAS) according to four different methods: 500 cells; one 40x-high power field (diameter $0.55 \mathrm{~mm}$ ); 2000 cells; and $2 \mathrm{~mm}^{2}$ or the entire tumor fragment(s) if this criterion was not fulfilled in BS. Mitoses were counted in $2 \mathrm{~mm}^{2}$ or the entire fragment(s) in BS, whereas necrosis was dichotomized as absent or present (whatever extent). Statistical analysis was according to Mann-Whytney and Kruskal-Wallis tests.

Results: There were 9 typical carcinoids (TC), 6 atypical carcinoids (AC), 2 large-cell neuroendocrine carcinomas (LCNEC) and 4 small cell carcinomas (SCC), in 13 females and 9 males. Twelve of them were in stage I (6 TC, 3 AC, 1 LCNEC and 2 SCC), 4 in stage II (2 TC, 1 AC and 1 SCC), and 5 in stage III (1 TC, 2 AC, 1 LCNEC and 1 SCC). Pairwise comparison of BS and SS did not show significant differences in Ki-67 LI when dealing with 500 cells $(p=0.429)$, the entire $40 x$ field $(p=0.689), 2000$ cells $(p=0.689)$ and $2 \mathrm{~mm}^{2}(\mathrm{p}=0.936)$. Likewise, no significant differences in Ki-67 LI were observed when contrasting the four different categories in both BS and SS ( $p=0.988)$ or stratifying for histology in either type of material ( $\mathrm{p}=0.174$ to $\mathrm{p}=0.985$ ). However, $\mathrm{AC}$ tended to show higher Ki-67 LI in SS than in BS, although differences were not significant $(p=0.174)$ likely due to heterogeneous distribution of proliferating cells within individual tumors. In turn, mitoses were more easily identifiable in SS than in BS $(\mathrm{p}=0.003)$, whereas no differences were observed in the prevalence of necrosis $(\mathrm{p}=0.733)$.

Conclusions: $\mathrm{Ki}-67 \mathrm{LI}$ in BS was as accurate as SS according to all the four different methods under evaluation. Ki-67 LI was more versatile but not more time consuming than mitoses and thus could have potential relevance to prognosis and tumor grading when assessing lung NET in BS.

\section{Salivary Gland-Type Primary Tumors of the Lung: A Systematic} Review

Emilian Racila, Erin Maxfield. University of Minnesota, Minneapolis, MN

Background: Primary salivary gland tumors of the lung are rare entities that are difficult to recognize mainly due to the limited experience most pathologists have in diagnosing these neoplasms in the lung. There are few published reports addressing the diagnostic characteristics of these tumors. We sought to determine the most important epidemiologic, morphologic and immunophenotypic features of these lesions.

Design: We identified 62 patients from our facility who were diagnosed with lung adenoid cystic carcinoma (ACC) or mucoepidermoid carcinoma (MEC) since 1996 to present. All inside cases were reviewed and characterized based on their morphologic and immunophenotypic characteristics. In addition we reviewed 19 published single case reports and 8 retrospective multiple case studies from domestic and international sources spanning 1988 to present.

Results: Our study population comprised 50 patients with ACC and 12 patients with MEC. In the ACC group, there were $22(44 \%)$ primary lung tumors and $28(56 \%)$ metastatic ACC to the lung. The primary lung ACC comprised of 14 females $(28 \%)$ with a mean age of 47.4 years and 8 males $(16 \%)$ with the mean age of 56.8 years. The overall mean age at the time of primary lung ACC diagnosis was 50.8 years. Metastatic ACC to the lung included 15 females $(30 \%)$ with a mean age of 46.6 years and 13 males $(26 \%)$ with mean age of 48.7 years. The overall mean age at the time of ACC diagnosis was 47.6 years. In the MEC group, there were $8(67 \%)$ primary lung tumors and $4(33 \%)$ metastatic MEC to the lung. The primary lung MEC comprised of 3 females (25\%) with a mean age of 57 years and 5 males $(42 \%)$ with the mean age of 31 years. The overall mean age at the time of lung primary MEC diagnosis was 40.8 years. Metastatic MEC included 4 patients (33\%), all males, with a mean age of 69.8 years. There were no significant histologic or immunophenotypic differences between primary and metastatic ACC or MEC of the lung.

Conclusions: Although there are no significant differences in the histologic grade or immunophenotype of the tumors, we found epidemiologic differences related to gender and age at time of diagnosis between primary and metastatic ACC and MEC of the lung. Clinical outcome analysis for both lung ACC and MEC is currently in progress. A comprehensive interpretation of important morphologic and immunophenotypic features for diagnosis of primary lung ACC and MEC is presented.

1906 Combined High-Grade Neuroendocrine Lung Carcinomas: A Clinicopathologic Study of Surgical Resection Cases from a Single Institution

Maheshwari Ramineni, Annikka Weissferdt, Stephen G Swisher, Cesar A Moran, Neda Kalhor. M.D. Anderson Cancer Center, Houston, TX

Background: Combined High-Grade Neuroendocrine Lung Carcinomas (CHNLC) are mixed tumors composed of a mixture of high-grade neuroendocrine carcinoma with a non-neuroendocrine component (or) a combination of both high-grade neuroendocrine carcinomas. Data on clinico-pathologic characteristics of these tumors is limited as a vast majority present with advanced stage disease and are not resected. Here, we present our experience of C-HNLCs surgically resected at our institution over a 10 year span. 
Design: All surgical resection cases (excluding biopsies/referrals) with a diagnosis of C-HNLC, surgically resected at M.D. Anderson Cancer Center were retrieved by searching the pathology database. H\&E slides and accompanying immunohistochemistry slides were reviewed.

Results: A total of 11 cases were identified. Pre-operative diagnosis of C-HNLC with both components was established in only 1 of 11 patients with all other FNA/ biopsies consisting of either adenocarcinoma or neuroendocrine carcinoma component alone. The histology of combined tumors is listed in table1.

\begin{tabular}{|l|l|}
\hline Histology of C-HNLCs & Number of cases (11) \\
\hline Adenocarcinoma+LCNEC & 5 \\
\hline Adenocarcinoma+SCLC & 3 \\
\hline Squamous cell carcinoma+SCLC & 1 \\
\hline LCNEC+SCLC & 2 \\
\hline
\end{tabular}

The LCNEC component was dominant in 5 cases, adenocarcinoma in 4 cases, SCLC in 1 case; while equal proportions of both adenocarcinoma and LCNEC was noted in 1 case. 4 of 11 cases had metastatic nodal disease (all 4 were N1), with metastatic deposit demonstrating adenocarcinoma histology in 2 cases and SCLC in the other two. 3 patients had Stage I (IA: 2, IB:1), 6 patients had Stage II (IIA:4, IIB:2), and 2 patients had Stage III (IIIA:2) disease. Upon follow-up, 7 patients had no evidence of disease over a follow-up period ranging from 1-32 months; while 4 patients had disease progression due to local recurrence (or) distant metastasis over a follow-up period ranging from $18-58$ months. In patients that had disease progression, the time interval for recurrence/ metastasis ranged from 2-6 months.

Conclusions: There was a major discordance in the diagnoses between biopsies and resections, which translates to a potential impact on the optimal management of these tumors. Most frequent histologic combination in the primary tumor were LCNEC and adenocarcinoma, while the metastatic tumor had either SCLC or adenocarcinoma alone. There was no correlation between the histology/proportion of the dominant component in the primary tumor and metastatic tumor.

1907 Prognostic Significance of lincRNA-p21 in Non-Small Cell Lung Carcinoma (NSCLC)

Jose Ramirez, Joan J Castellano, Adela Saco, Jorge Moises, Ana Cordeiro, Ramon Marrades, Nuria Vinolas, Francisco Aya, Laureano Molins, Mariano Monzo, Alfons Navarro. Hospital Clinic, University of Barcelona, Barcelona, Spain; University of Barcelona, Barcelona, Spain; Hospital Clinic, Barcelona, Spain.

Background: The discovery that only $3 \%$ of human genome encodes protein highlighted the importance of non-coding RNAs (ncRNAs) ( $85 \%$ of the genome). Long ncRNAs (>200nt) are the most frequent type, but only a few of them have been associated with tumorigenesis. LincRNA-p21 is a long ncRNA located in chromosome 6, upstream of $\mathrm{p} 21$. It is transcriptional activated by $\mathrm{p} 53$ and HIF $1 \alpha$ and it has multiple functions including regulation of $\mathrm{p} 53$-dependent apoptosis and Warburg effect. We have investigated the role of lincRNA-p21 in NSCLC prognosis.

Design: Tumor and normal tissue from 91 lungs resected as part of the therapy for NSCLC were studied: $78 \%$ were male; $59.3 \%$ were stage I, $20.9 \%$ stage II and $19.8 \%$ stage III; $53.8 \%$ were Adenocarcinoma (ADK); $22 \%$ harbor p53 mutations; $30.8 \%$ received adjuvant treatment after surgery. LincRNA-p21 was determined by Real time

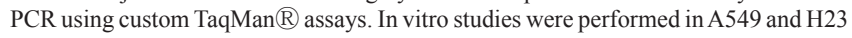
cell lines. Immunohistochemistry was performed in FFPE tissues.

Results: LincRNA-p21 was downregulated in tumor tissue in comparison to paired normal tissue $(p=0.006)$. Association neither with main clinical characteristics nor with p53 mutational status and lincRNA-p21 was observed. The correlation of lincRNA-p21 levels and TTR and OS showed that in the ADK subgroup high levels of lincRNA-p21 were associated with shorter TTR $(p=0.02)$ and shorter OS $(p=0.019)$. In the multivariate analysis including clinical variables, lincRNA-p21 emerged as independent prognostic factor for TTR (OR: 5.2, 95\%CI: 1.7-15.6; $\mathrm{p}=0.003$ ) and $\mathrm{OS}(\mathrm{OR}: 10.15,95 \% \mathrm{CI}$ : 2.2-47.1; $\mathrm{p}=0.002$ ). To investigate the role of lincRNA-p21 in tumor progression we studied their implication in angiogenesis. We observed that in A549 and H23 cell lines, lincRNA-p21 knockdown in hypoxic conditions reduced significantly VEGFA levels (49 and 39\% of reduction respectively). The comparison of microvessel density by Immunohistochemistry of high vs low lincRNA-p21 cases, showed that patients with high lincRNA-p21 had higher microvessel density in the tumor $(\mathrm{p}=0.0022)$.

Conclusions: High levels of lincRNA-p21 are associated with worse prognosis in patients with lung Adenocarcinoma through regulation of angiogenesis.

(Grants: FIS 12/405; AECC-Catalunya 2013)

1908 Low Expression of SerpinB2 Is Associated with Reduced Survival in Lung Adenocarcinomas

Maria Ramnefjell, Christina Aamelfot, Lars Helgeland, Lars A Akslen. University of Bergen, Bergen, Norway; Haukeland University Hospital, Bergen, Norway.

Background: Lung cancer is a leading cause of cancer related deaths worldwide, and finding new prognostic biomarkers are of utmost importance. Previous studies have shown that the plasminogen activator inhibitors SerpinB2 and Neuroserpin and the adhesion molecule L1CAM have an impact on disease progression and development of metastasis, especially to the brain. Here, we examined whether expression of these markers was associated with patient prognosis, site of metastasis or other clinicopathologic characteristics in non-small cell lung cancer (NSCLC).

Design: The material consists of surgical specimens from 438 NSCLC patients treated during 1993-2010 at Haukeland University Hospital, Bergen, Norway. Sections from selected tumor blocks were stained with antibodies to SerpinB2, Neuroserpin, and L1CAM. There were 277 men and 161 women, with a mean age at surgery of 66 years
(213 adenocarcinomas, 135 squamous cell carcinomas and 90 other carcinomas). Mean observation time was 60 months (range $0-254$ months). 118 patients were fully recovered, 177 died of lung cancer, and 123 died from other causes.

Results: Low expression of SerpinB2 was associated with reduced lung cancer specific survival $($ LCSS $)(p=0.017)$ in adenocarcinomas, but not in other major histological subtypes. There were no associations with survival for Neuroserpin or L1CAM.

Neuroserpin was associated with tumor diameter, tumor grade, necrosis, and pleural invasion, and L1CAM with tumor diameter, tumor grade, vessel invasion and necrosis $(\mathrm{p}<0.05$ for all).

In multivariate survival analysis of adenocarcinomas where tumor diameter, tumor grade, vessel invasion, necrosis, inflammation, stage and SerpinB2 expression were included, SerpinB2 demonstrated independent prognostic significance for LCSS (HR $1.6(95 \%$ confidence interval $1.05-2.48, \mathrm{p}=0.027))$.

Conclusions: Low expression of SerpinB2 in lung adenocarcinomas was associated with reduced LCSS and was an independent prognostic factor in multivariate analysis in our cohort. In contrast to previous findings by others, we did not find an impact of Neuroserpin and L1CAM on survival.

Low expression of SerpinB2 could be a biomarker for prognosis in lung adenocarcinomas and should be studied in other cohorts.

\section{Evaluation of Genomic Complexity in Next-Generation Sequencing} of T1 vs Stage IV Non-Small Cell Lung Cancer

Amanda J Redig, Christine Lydon, Pasi A Janne, Lynette M Sholl. Dana-Farber Cancer Institute, Boston, MA; Harvard Medical School, Boston, MA; Brigham and Women's Hospital, Boston, MA

Background: Targeted next-generation sequencing (NGS) is increasingly utilized in the diagnostic evaluation and therapeutic management of lung cancer. The clinical outcomes vary widely depending on disease stage, yet differences in the genomic profiles of early-stage versus advanced tumors have not been well-described.

Design: NGS data for NSCLC tumors evaluated between July 2013 and October 2014 was reviewed. A cohort of 23 resected Stage I tumors including 20 adenocarcinomas (ACA) and 3 minimally invasive adenocarcinomas was identified, with a 2:1 comparison to a cohort of Stage IV ACA $(n=46)$. Total numbers of single nucleotide variants (SNV) and copy number variants (CNV), along with the presence of selected mutations (EGFR, KRAS, TP53, STK11) was determined for each tumor.

Results: The median patient age in the $\mathrm{T} 1$ cohort was 65 years compared to a median age of 61 years in the Stage IV cohort. $61 \%$ of the tumors in both cohorts were from female patients. $5 / 23$ patients $(22 \%)$ in the T1 cohort were never/light smokers compared to $12 / 46(26 \%)$ in the Stage IV cohort.The median number of SNVs in T1 tumors was 5 , with $5 / 23$ tumors $(22 \%)$ containing $>10$ variants. In Stage IV tumors, the median number of SNVs was 7.5 , with $14 / 46$ tumors $(30 \%)$ containing $>10$ variants. T1 tumors had a median of 3 gene-level CNVs compared to a median of 18 in Stage IV tumors (Student's T test, $\mathrm{p}=0.001)$. KRAS mutations were identified in $8 / 23(35 \%) \mathrm{T} 1$ tumors vs. $23 / 46(50 \%)$ stage IV tumors $(\mathrm{p}=0.3)$. EGFR mutations were identified in $3 / 23(13 \%)$ T1 tumors vs. $7 / 46(15 \%)$ stage IV tumors (NS). $4 / 23(17 \%)$ of T1 tumors and $22 / 46(48 \%)$ of Stage IV tumors contained a TP53 mutation $(\mathrm{p}=0.02)$ while $6 / 23$ (26\%) of T1 tumors and 8/46 (17\%) of Stage IV tumors contained an STK11 mutation. Conclusions: The frequency of mutations in major oncogenic drivers does not vary significantly between T1 and stage IV ACA, consistent with the observation that EGFR and KRAS mutations are early events in lung tumorigenesis. However, advanced stage tumors are significantly more likely to harbor TP53 mutations and higher levels of $\mathrm{CNV}$ consistent with genomic instability, suggesting that these are later events in lung ACA progression.

1910 Next-Generation Sequencing of Pulmonary Large Cell Neuroendocrine Carcinoma Reveals Clinically-Relevant Subsets with Small Cell Carcinoma-Like and Non-Small Cell Carcinoma-Like Molecular Profiles

Natasha Rekhtman, Jarushka Naidoo, Maria Pietanza, Matthew D Hellmann, Arshi Arora, Ronglai Shen, Darragh F Halpenny, Helen Won, Michael F Berger, Shaozhou Tian, John T Poirier, Paul K Paik, Andre Moreira, Charles M Rudin, William D Travis, Marc Ladanyi. Memorial Sloan Kettering Cancer Center, New York, NY.

Background: Pulmonary large cell neuroendocrine carcinoma (LCNEC) shares with small cell lung carcinoma (SCLC) highly aggressive clinical behavior and neuroendocrine phenotype; yet, whether LCNEC is biologically related to SCLC versus non-SCLC (NSCLC) remains unclear, contributing to the uncertainty regarding optimal clinical management. Here, we compared genomic alterations in LCNEC to those in SCLC and other major lung cancer types.

Design: LCNEC $(n=45)$ tumor/normal pairs underwent next-generation sequencing of 241 cancer genes (MSK-IMPACT), mass-spectrometry genotyping $(\mathrm{n}=62)$, and detailed histologic, immunohistochemical and clinical characterization. Genomic data were compared to same-platform analysis SCLC, NSCLC, and carcinoids.

Results: Commonly alerted genes in LCNEC included TP53 (78\%), RB1 (38\%), STK11 (33\%), KEAP1 (31\%) and KRAS (22\%). Genomic profiles segregated LCNEC into three subsets: SCLC-like ( $\mathrm{n}=18)$, characterized by $T P 53+R B 1$ co-alteration, $M Y C L$ and SOX2 amplifications, and PTEN mutation/loss; NSCLC-like (n=25) - lacking TP53+RB1 co-alteration and instead exclusively harboring adenocarcinoma-type mutations (STK11, $K R A S, M A P 2 K 1)$; and carcinoid-like (n=2), characterized by MEN1 mutations and low total mutation burden. SCLC-like subset exhibited higher proliferation rates $(P<0.0001)$, a trend toward lower recurrence-free survival, greater chemosensitivity $(P=0.03)$, and fewer potentially-targetable genomic alterations $(P=0.03)$, whereas NSCLC-like tumors revealed low-level adenocarcinoma-type differentiation marker expression. Notably, compared to conventional adenocarcinoma, NSCLC-like LCNEC harbored more frequent alterations in key neurogenesis regulators, including NOTCH family $(P=0.005)$. 
Conclusions: LCNEC is a heterogenous category, representing a histologic convergence of tumors genomically and clinicopathologically related to SCLC, NSCLC and rarely highly proliferative carcinoids. Our findings suggest that recognition of these molecular subsets may inform the classification and management of LCNEC patients.

1911 Correlation of Histologic and Radiologic Response to Neoadjuvant Treatment of Thymic Epithelial Tumors

Anja C Roden, Marie Christine Aubry, Eunhee S Yi, Sarah M Jenkins, Yolanda I Garces, Randolph S Marks, Stephen D Cassivi, Geoffrey B Johnson. Mayo Clinic, Rochester, MN

Background: Neoadjuvant treatment (NAT) might increase resectability of thymic epithelial tumors (TET). No standardized pathologic

grading scheme for tumor response is available in TET. Also, it is unclear whether radiologic treatment response can predict pathologic response.

Design: Pts. with unresectable TET who underwent NAT before surgery at our institution (1942-2014) were included. Pre- and post-NAT CT scans were reviewed by a thoracic radiologist. Response Evaluation Criteria in Solid Tumors (RECIST) criteria v1.1 (Byrne modification) were applied. Pre- and post-NAT specimens were reviewed by 3 thoracic pathologists. The tumor response grade (TRG) was based upon Mandardgrading (1994) (table)

Results: 49 pts (29 men) [median age, 47.6 yrs (range 25.0 - 77.2)] were included. Results of NAT response are summarized in table.

TRG distribution [n (row \%)] within each characteristic.

\begin{tabular}{|c|c|c|c|c|c|}
\hline TRG & 1 & 2 & 3 & 4 & 5 \\
\hline Histology & $\begin{array}{l}\text { Complete } \\
\text { pathologic } \\
\text { response-no } \\
\text { viable tumor }\end{array}$ & $\begin{array}{l}\text { Rare residual } \\
\text { viable tumor } \\
\text { cells scattered } \\
\text { through } \\
\text { fibrosis }\end{array}$ & $\begin{array}{l}\text { Increase in } \\
\text { \# of residual } \\
\text { tumor cells; } \\
\text { fibrosis } \\
\text { predominant }\end{array}$ & $\begin{array}{l}\text { Residual } \\
\text { viable } \\
\text { tumor } \\
\text { outgrowing } \\
\text { fibrosis }\end{array}$ & $\begin{array}{l}\text { No } \\
\text { regressive } \\
\text { changes }\end{array}$ \\
\hline \multicolumn{6}{|l|}{ Diagnosis } \\
\hline $\begin{array}{l}\text { Thymoma } \\
(\mathrm{N}=30)\end{array}$ & 0 & $1(3)$ & $5(17)$ & $9(30)$ & $15(50)$ \\
\hline $\begin{array}{l}\text { Thymic } \\
\text { carcinoma } \\
(\mathrm{N}=19)\end{array}$ & $3(16)$ & $4(21)$ & $8(42)$ & $3(16)$ & $1(5)$ \\
\hline \multicolumn{6}{|l|}{$\begin{array}{l}\text { Radiologic } \\
\text { Response } \\
(\mathrm{N}=24)\end{array}$} \\
\hline $\begin{array}{l}\text { Stable Disease } \\
(\mathrm{N}=13)\end{array}$ & 0 & $1(8)$ & $4(31)$ & $5(39)$ & $3(23)$ \\
\hline $\begin{array}{l}\text { Partial } \\
\text { Response } \\
(\mathrm{N}=11)\end{array}$ & $3(27)$ & $2(18)$ & $5(46)$ & $1(9)$ & 0 \\
\hline \multicolumn{6}{|l|}{$\begin{array}{l}\text { Neoadjuvant } \\
\text { Treatment }\end{array}$} \\
\hline $\begin{array}{l}\text { Chemotherapy } \\
(\mathrm{N}=22)\end{array}$ & 0 & $1(5)$ & $7(32)$ & $9(41)$ & $5(23)$ \\
\hline $\begin{array}{l}\text { Radiation } \\
(\mathrm{N}=13)\end{array}$ & 0 & 0 & $2(15)$ & $2(15)$ & $9(69)$ \\
\hline $\begin{array}{l}\text { Chemoradiation } \\
(\mathrm{N}=14)\end{array}$ & $3(21)$ & $4(29)$ & $4(29)$ & $1(7)$ & $2(14)$ \\
\hline
\end{tabular}

$67 \%$ pts were completely resected. Fibrosis was the most common histologic finding in post-NAT thymomas (T) ( $80 \%$ of cases) and thymic carcinomas (TCa) (100\%); necrosis was uncommon in T $(13 \%)$ in contrast to TCa $(53 \%)(\mathrm{p}=0.008)$. Agreement for TRG by 3 reviewers was good (Krippendorff $\alpha, 0.838$ ). By imaging $(\mathrm{N}=24)$, partial response and larger reduction in tumor diameter and volume were associated with lower TRG ( $p=0.0093, p=0.0042, p=0.0021$, respectively) and less \% viable tumor $(p=0.0041$, $\mathrm{p}=0.0034,0.0019)$. TRG and radiologic change in tumor diameter correlated (Spearman correlation coefficient, 0.60$)$. Radiologic change in tumor diameter and volume reasonably predicted pathologic TRG of $3-5$ vs TRG 1,2 (AUC $0.74,0.71$, respectively). Conclusions: Our proposed histologic TRG for TET appears easy and reproducible and correlates with radiologic response. Radiologic response is useful to predict pathologic response.

1912 Comprehensive Analysis of EGFR Mutation Status in Lung Adenocarcinoma Combined with Nonadenocarcinoma Component

Mee Sook Roh, Hyoun Wook Lee. Dong-A University College of Medicine, Busan, Korea; Samsung Changwon Hospital, Sungkyunkwan University School of Medicine, Changwon, Korea.

Background: Lung adenocarcinomas (ADs) are found to be composed of a mixture of nonadenocarcinoma (non-AD) components. We analyzed EGFR mutation at each component in $\mathrm{AD}$ combined with non-AD component to investigate the relationship between $E G F R$ mutation status and the histologic type and volume of non-AD component.

Design: We analyzed 39 Korean patients with surgically resected lung cancer (19 adenosquamous carcinoma (ADSQ), 9 neuroendocrine carcinoma combined with AD (NE-AD; 3 small cell lung carcinoma combined with AD (SCLC-AD) and 6 large cell neuroendocrine carcinoma combined with AD (LCNEC-AD)), and 11 pleomorphic carcinoma with AD (PC-AD). After genomic DNA was extracted from separately microdissected paraffin-embedded tissues, mutation analysis of $E G F R$ was performed using peptide nucleic acid-clamp real-time PCR-based assay in both AD and non-AD components.
Results: The detection rate for EGFR mutation was 31.6\% (6 cases: 1 in exon 19 and 5 in exon 21) in 19 ADSQ patients, 11.1\% (1 SCLC-AD case: 1 in exon 19) in 9 NE-AD patients, and 9.1\% (1 case: 1 in exon 19$)$ in PC-AD patients. Identical EGFR mutations were found in both AD and non-AD components in all 6 ADSQ cases and 1 SCLC-AD case, whereas EGFR mutation was detected in AD component, but not in non-AD component in PC-AD. Although the volume of AD tended to be larger $(\geq 50 \%)$ in tumor with EGFR mutation than in tumor without $E G F R$ mutation, no statistical significance was found.

\begin{tabular}{|l|l|l|l|l|}
\hline $\begin{array}{l}\text { Case with } E G F R \\
\text { mutation }\end{array}$ & Histology & $\begin{array}{l}\text { Volume } \\
\text { ratio } \\
\text { of AD (\%) }\end{array}$ & $\begin{array}{l}\text { EGFR mutation } \\
\text { in AD }\end{array}$ & $\begin{array}{l}\text { EGFR mutation in } \\
\text { non-AD }\end{array}$ \\
\hline 1 & ADSQ & 90 & Exon 21, L858R & Exon 21, L858R \\
\hline 2 & ADSQ & 80 & Exon 21, L858R & Exon 21, L858R \\
\hline 3 & ADSQ & 80 & Exon 21, L858R & Exon 21, L858R \\
\hline 4 & ADSQ & 60 & Exon 21, L858R & Exon 21, L858R \\
\hline 5 & ADSQ & 40 & Exon 19 deletion & Exon 19 dletion \\
\hline 6 & ADSQ & 30 & Exon 21, L858R & Exon 21, L858R \\
\hline 7 & SCLC-AD & 70 & Exon 19 deletion & Exon 19 deletion \\
\hline 8 & PC-AD & 60 & Exon 19 deletion & Wild \\
\hline
\end{tabular}

Conclusions: We clarified that a subset of non-AD components contains $E G F R$ mutations. In particular, ADSQ had a relatively high frequency of $E G F R$ mutation. Thus, prospective identification of EGFR mutations in ADSQ, combined NE carcinoma and $\mathrm{PC}$ before the initiation of treatment is an essential step to identify which patient might benefit from targeted therapies, especially in advanced tumors which are not resected and where only small biopsies are available for molecular testing.

1913 Lung Adenocarcinoma with Mucin-Related Antigen 4 Expression Is Associated with the Smoking Status and Poor Prognosis

Mariyo Rokutan, Akihiko Yoshizawa, Hironori Haga. Kyoto University Hospital, Kyoto, Japan.

Background: Mucin-related antigen 4 (MUC4) is a transmembrane glycoprotein that functions in the cell growth signaling pathway. The expression of MUC4 has been studied in malignancies of various organs and is associated with a poor prognosis. However, the significance of MUC4 expression in lung cancer is unclear. The aim of this study was to analyze MUC4 expression in resected lung adenocarcinomas by using immunohistochemistry (IHC) to define the clinicopathological characteristics better. Design: Immunohistochemistry analysis of MUC4 was performed by using tissue microarray slides of 348 lung adenocarcinomas. Scoring was based on the distribution and intensity of staining ( $\mathrm{H}$ score). A positive case was determined by considering an $\mathrm{H}$ score of 51-300 and a negative case by considering an $\mathrm{H}$ score of $0-50$. The association between MUC4 expression and the following clinicopathological parameters was evaluated: sex; age; smoking status; tumor stage; tumor grade; lymph-vascular invasion; pleural invasion; expression of MUC5B, MUC5AC, MUC6, TTF-1, Cdx2, and MUC2 (based on IHC); and the mutation status of EGFR, KRAS, and HER2. Moreover, the survival rates were calculated using the Kaplan-Meier method.

Results: The expression of MUC4 was positive in 97 (27.9\%) tumors. Most patients with MUC4 expression were men $(\mathrm{p}=0.001)$ and smokers $(\mathrm{p}=0.014)$. Moreover, MUC4 expression was significantly associated with solid adenocarcinoma $(\mathrm{p}<$ $0.001)$, high histological tumor grade $(\mathrm{p}=0.001)$, and vascular invasion $(\mathrm{p}=0.001)$, whereas it was not associated with age, tumor size, tumor stage, lymphatic invasion, pleural invasion, and IHC expression of MUC5B, MUC5AC, MUC6, TTF-1, Cdx2, and MUC2. The expression of MUC4 had a significant inverse correlation with EGFR mutation $(\mathrm{p}=0.002)$, whereas its expression was not associated with mutations in KRAS or HER2. Patients with MUC4 expression had a significantly worse prognosis, with a $64.7 \%$ overall survival rate at 5 years, compared to patients without MUC4 expression $(\mathrm{p}=0.023)$

Conclusions: The present study showed that lung adenocarcinomas with MUC4 expression tend to develop in male smokers, with a high grade tumor, and with the EGFR wild-type gene. Moreover, these patients tended to have a poorer prognosis. Taken together, lung adenocarcinoma with MUC4 expression can be a distinct phenotype caused by smoking.

1914 Next Generation Sequencing Reveals Genomic Heterogenity of ALK Fusion Breakpoints in Non -Small Cell Lung Cancer

Jason N Rosenbaum, Julie Branson, Catherine E Cottrell, John D Pfeifer, Shashikant Kulkarni, Eric J Duncavage. Washington University School of Medicine, Department of Pathology and Immunology, St. Louis, MO.

Background: In non--small cell lung cancer (NSCLC), the canonical EML4--ALK inversion results in a fusion protein with a constitutively active ALK kinase, which is associated with response to targeted inhibitors such as crizotinib. Clinically, anti-ALK therapy is often initiated based on evidence of an $A L K$ rearrangement,- generally detected by fluorescent in situ hybridization (FISH) of interphase cells in FFPE tissue sections. However, FISH cannot differentiate between different $A L K$ partner genes or non-canonical breakpoints, raising the possibility that not all $A L K$ rearrangements are detected, and that not all detected $A L K$ fusions may be responsive to targeted inhibitors. Indeed, "ALK positive" NSCLC has only a $60 \%$ objective response rate to ALK inhibitors. In this study we sought to determine if next generation sequencing (NGS) targeting DNA-level translocations could further define $A L K$ rearrangements. Design: From a set of 1532 cases evaluated for an $A L K$ rearrangement by interphase FISH, we identified 29 rearranged cases, of which 11 had concurrent clinical DNA NGS sequencing performed as part of routine patient care (via a hybrid capture based 
approach using paired $2 \times 101 \mathrm{bp}$ reads). The sequencing included probes targeting $A L K$ introns 16-22 in addition to other cancer-related genes. Rearrangements identified using the Breakdancer and ClusterFast software packages.

Results: All 11 cases positive for $A L K$ rearrangement by FISH showed evidence of the translocation by sequencing. Non--canonical breakpoints and rearrangement partners were identified in 4 of 11 cases ( $36 \%$ ). In two of 4 cases the $A L K$ rearrangement involved intergenic, noncoding DNA on chromosome 2; in the remaining 2 cases $S P D Y A--A L K$ and $A S X L 2--A L K$ fusions were detected. In the $A S X L 2-A L K$ fusion, despite having the canonical $A L K$ intron 19 breakpoint, the genes are in opposing transcriptional orientation, suggesting no functional $A L K$ kinase domain is transcribed. Overall, sequencing data predict three of these four translocations to result in a non-functional fusion protein.

Conclusions: NGS-based testing demonstrates substantial genomic heterogeneity in $A L K$-rearranged NSCLC. NGS-based testing can resolve novel partner genes and noncanonical breakpoints, which may impart a different therapeutic response to targeted inhibitors. FISH testing for "ALK positive" lung adenocarcinoma underestimates the heterogeneity among $A L K$ translocations.

1915 Usefulness and Limitation of Endobronchial Ultrasound Transbronchial Needle Aspiration (EBUS) Specimen for PD-L1 Companion Diagnosis in Lung Cancer

Rie Sakakibara, Noriko Motoi, Hironori Ninomiya, Kentaro Inamura, Makoto Nishio, Yuichi Ishikawa. The Cancer Institute, Japanese Foundation for Cancer Research, Koto, Tokyo, Japan; Cancer Institute Hospital, JFCR, Koto, Tokyo, Japan.

Background: For personalized cancer therapy, it is required to establish a suitable companion diagnostic strategy for an adequate molecular target. In lung cancer, it is often difficult to obtain enough tumor sample by a conventional transbronchial biopsy. For example, in order to enroll in clinical trial with programmed cell death-ligand 1 (PD-L1) target therapy, it is required to submit enough tumor (more than 100 tumor cells). In this study, we studied the potential usefulness of an endobronchial ultrasound transbronchial needle aspiration (EBUS) system which is a novel minimally invasive biopsy method recently introduced in Japan for companion diagnosis of PD-L1.

Design: We collected consecutive 100 primary lung cancer cases diagnosed by EBUS procedures between 2013 and 2014. We evaluated numbers of tumor cells and total nuclear cells in each specimen. First, we counted by eye-ball on HE stained slides. Then, we calibrated tumor cells by combination of immunohistochemical tumor markers ( TTF1 as adenocarcinoma (Ad), p40 as squamous cell carcinoma (SqCC) and synaptophysin as neuroendocrine carcinoma (NEC) markers) with an automated image calibration system using an Aperio Image Scope (Leica systems). We also assessed PDL1 immunohistochemical (IHC) expression in both EBUS and corresponding surgical resected specimens. The PD-L1 membranous IHC expressions were scored as 0,1,2,3. Results: Pathological diagnoses of 100 cases were as following; $53 \mathrm{Ad}, 17 \mathrm{SqCC}$ and $27 \mathrm{NEC}$. The median (and range) number of tumor cells in all/ $\mathrm{Ad} / \mathrm{SqCC} / \mathrm{NEC}$ cases were $1337(8-6000) / 1140(81-6910) / 878(158-3835) / 4620(146-60000)$ by eye-ball counting, and $1851(0-59120) / 1531(1-44224) / 4243(0-17065) / 3157(11-59120)$ by automated image calibration. The eye-ball counting and automated image calibration showed good correlation. All but one cases $(99 \%)$ had sufficient numbers of tumor cell for enrollment of PD-L1 clinical study. The results of PD-L1 IHC score in EBUS and surgical specimen showed good concordance. All EBUS case with corresponding surgical resected specimen resulted more than score 1 in this study. The PD-L1 IHC expression degree (proportion and/or intensity) was heterogeneous in surgically resected specimen.

Conclusions: Our study revealed EBUS specimens can be useful for companion diagnosis of PD-L1 in case with IHC positive cases, but it should be mentioned as a critical limitation of possibility of false negative cases due to intratumoral heterogeneity.

\section{Detection of BRAF Mutation in Lung Adenocarcinoma by Next-} Generation Sequencing: Beyond V600E Mutation

Kevan Salimian, Roghayeh Fazeli, Gang Zheng, Zahra Maleki. The Johns Hopkins Hospital, Baltimore, MD.

Background: Molecular identification of driver mutations in lung adenocarcinomas (ACA) has changed the landscape of management. EGFR mutation and ALK translocation in lung ACA predict favorable responses to targeted therapy and have been fairly well studied. BRAF mutations have also been described in a small subset of lung ACA. We devised this study to analyze the histologic and cytomorphologic features of BRAF-mutated lung ACA, along with the clinical characteristics of patients presenting with this entity.

Design: We performed a retrospective review of all surgical pathology and cytopathology specimens at Johns Hopkins from January 2013 to January 2015 that contained BRAF-mutated lung ACA detected by next-generation sequencing (NGS). Patient demographics, clinical history, ACA morphology, and molecular studies were assessed and stratified based on BRAF mutational status (V600E vs non-V600E).

Results: Ten patients harbored V600E mutations and 26 patients carried non-V600E mutations. The mean age was similar for both groups. V600E mutation was more common in females $(90 \%)$. Smoking showed strong correlation with BRAF mutation in both groups ( $90 \%$ or greater). While both groups shared a variety of morphologic patterns, lepidic and mucinous were more common in the non-V600E group. Both groups showed variable differentiation and tumor staging. Both groups were immunoreactive for TTF-1 and Napsin A but negative for P40. The V600E group harbored an AKT mutation in two cases and a PIK3CA mutation in one case. The non-V600E group contained a KRAS mutation in four cases and a PIK3CA mutation in one case. Non-V600E mutations included D594 ( $\mathrm{n}=7)$, G469 ( $\mathrm{n}=5), \mathrm{G} 466(\mathrm{n}=4), \operatorname{K} 601 \mathrm{E}(\mathrm{n}=3), \mathrm{G} 596(\mathrm{n}=2)$, $\mathrm{G} 464 \mathrm{~V}(\mathrm{n}=2)$, and one each of Q609L, E611Q, and T4401. No ALK rearrangement was detected in either group in available cases.
Conclusions: Mutated-BRAF lung ACA tend to arise in older patients with the vast majority being current or former smokers. The V600E mutation was present in slightly less than $30 \%$ of cases which could have therapeutic promise. BRAF mutations were infrequently found in combination with other common lung ACA driver mutations. The histologic grade and architecture of these tumors varied significantly. NGS has made it possible to detect more BRAF mutations in addition to V600E. Further investigation is warranted to evaluate the role of non-V600E mutations in patients with lung ACA.

1917 Prevalence of ALK and ROS1 Rearrangements in Mexican Patients with Lung Adenocarcinoma

Sergio Sanchez, Silvia Montilla, Pamela R Matias, Julieta Garcia, Ivan R Gonzalez. Hospital Angeles Puebla, Puebla, Mexico.

Background: Screening of anaplastic lymphoma tyrosine kinase (ALK) and c-ros oncogene 1 (ROSI) gene fusions in non-small cell lung cancer (NSCLC) patients enables the identification of the patients likely to benefit from MET/ALK inhibitor. The aim of this study was to evaluate the prevalence of $A L K$ and ROS1 rearrangements in Mexican NSCLC patients, which has not been reported earlier, and to study the association between these molecular alterations and clinicopathological characteristics in these patients.

Design: The study included samples from 62 patients diagnosed with advanced NSCLC. ALK fluorescence in-situ hybridization (FISH) was performed using the Vysis ALK Break Apart Rearrangement Probe Kit, and ROS1 FISH assay used the Cytocell ROS1 Breakapart Probe; FISH-positive cases were defined as those with more than $15 \%$ breakapart signals or isolated red signals in 100 cells. Clinicopathological characteristics, including sex, age, TNM stage, tumor differentiation, smoking status and histological subtypes were analyzed.

Results: A total of 60 formalin-fixed paraffin-embedded tumor tissue specimens from Mexican NSCLC patients were screened for $A L K$ and ROSI translocations by FISH; of which there were $28(46.6 \%)$ men and $32(53.4 \%)$ women with a median age of 62 years. We diagnosed $2 \mathrm{ALK}$-rearranged adenocarcinomas (3.3\%) and $2 \mathrm{ROSl}$-altered adenocarcinomas $(3.3 \%)$, with $56-74 \%$ and $60-72 \%$ rearranged nuclei, respectively. All the positive cases were at an advanced stage, arising in never or light smokers, and were classified in $57(95 \%)$ adenocarcinomas, $1(1.6 \%)$ epidermoid, and $2(3.3 \%)$ presented undifferentiation.

Conclusions: The incidence of $A L K$ and ROS1 mutations was of 3.3\% (with a 95\% CI were $[0.39 \%-11.17 \%]$ ) in this Mexican population and is similar to what has been observed in other Western populations. Our results show that a screening algorithm that includes $A L K$ and $R O S I$ testing by FISH is pertinent in advanced lung adenocarcinomas, since the combined prevalence of positive cases in this selected population reaches $6.6 \%$. This highlights the importance of mutational profiling to identify driver genetic alterations in a clinical setting in order to facilitate personalized lung cancer medicine.

1918 Validation of a Simplified Approach to Detect ALK Translocations in Lung Cancer Samples by FISH

Hans-Ulrich Schildhaus, Elke Binot, Jurgen Wolf, Reinhard Buettner, Katja Schmitz. University Hospital Goettingen, Goettingen, Germany; University Hospital Cologne, Cologne, Germany; Center for Integrated Oncology, Cologne-Bonn, Germany; Lung Cancer Group Cologne, Cologne, Germany; Comprehensive Cancer Center (G-CCC) and Lungentumorzentrum (LTZ), Goettingen, Germany.

Background: ALK rearrangements constitute a major therapeutic target in pulmonary adenocarcinomas. FISH still represents the gold standard for the detection of these genetic changes. In this study we aimed at investigating whether speed and accuracy of ALK FISH can be improved if a triple color combined fusion/break apart approach is applied.

Design: 366 tumors were prospectively analyzed, among them 21 ALK positive carcinomas. All tumors were hybridized with two different ALK FISH probes, Vysis LSI $A L K$ Dual Color Break-Apart Rearrangement Probe and ZytoLight ${ }^{\circledR}$ SPEC $A L K /$ EML4 TriCheck ${ }^{\mathrm{TM}}$ Probe. The latter tricolor probe set contains an additional probe for the detection of EML4 resulting in orange/blue fusion signals in the case of an EML4/ ALK fusion (beside conventional orange/green break aparts or isolated 3' orange signals). Cases were evaluated by one pathologist at different times blinded for the result of the other probe.

Results: Both probe sets correctly identified all $A L K$ positive tumors resulting in an overall $100 \%$ sensitivity and specificity of the ZytoLight probe compared the Vysis one (if 50 tumor cells were evaluated in both assays). The tricolor approach allowed a better evaluation of tumor cells with close distances between green 5 ' and orange 3' signals ( $<2$ signal diameters). We could show that a significant minority of these signal patterns represent true EML4/ALK inversions since we could demonstrate blue/orange fusion signals. Therefore, we evaluated whether the accuracy of this test can be kept if only 20 tumor cells are analyzed. For this approach, we defined diagnostic categories as follows: i) if 0 or 1 out of 20 tumor cells show an aberrant pattern (break aparts or isolated orange signals, both with or without colocalization of blue signals) the case was considered negative; ii) cases with $>=6$ tumor cells were considered positive for ALK rearrangement; iii) cases with $2-5$ aberrant tumor cells were considered equivocal and a re-test of additional 30 cells was carried out, resulting in a total of 50 cells. By applying this approach all tumors were correctly classified. Only 5 tumors $(1 \%)$ were equivocal and required re-test.

Conclusions: We established and validated a novel enumeration approach for $A L K$ FISH. 
1919 Programmed Cell Death 1 Biomarker Testing: RNA and Protein Assessment

Brandon S Sheffield, Regan Fulton, Katy Milne, Celine Jacquemont, Brad H Nelson, Cheryl Ho, Allen M Gown, Diana NIonescu. University of British Columbia, Vancouver, Canada; PhenoPath, Seattle, WA.

Background: Targeted inhibition of the programmed cell death 1 (PD1) axis is a promising approach to cancer treatment. This may be aided by the a priori identification of responders by pathology laboratories. PD1 ligand (PD-L1) overexpression can be identified by IHC; however, existing reports regarding the utility of PD-L1 IHC as a biomarker are conflicting. These uncoordinated efforts are mired with heterogeneity in IHC methodologies, assessment, and reporting, with no identified gold standard for calibration. Concurrent studies by our group have identified $P D-L 1$ expression as a candidate reference to standardize IHC approaches. Here, we explore RNA in situ hybridization (ISH), a glass-based, light microscopic technique, as a useful molecular adjunct to PD-L1 biomarker testing in lung adenocarcinoma.

Design: A tissue microarray containing surgically resected non-small cell, non squamous, lung carcinoma was stained for PD-L1 using SP142 (Spring Biosciences), E1L3N (Cell Signaling Technology), and RBT-PDL1 (BioSB) primary antibodies. RNA-ISH was performed using RNA Scope (Advanced Cell Diagnostics). Both methods were scored in tumor cells and semi-quantified using combined intensity and proportion scores.

Results: 80 cases were evaluated. Immunoreactivity was identified in $29(36 \%)$ cases by SP142, $19(24 \%)$ by E1L3N, and 29(36\%) by RBT-PDL1; significant RNA signal was identified in $18(23 \%)$ cases. One (1\%) case was identified with positive RNA signal and no immunoreactivity, $3(4 \%)$ cases showed significant immunoreactivity with no RNA signal. By linear regression analysis, SP142, E1L3N, and RBT clones showed high correlation with RNA ISH $\left(\mathrm{R}^{2}=0.51,0.47,0.55\right.$ respectively $)$.

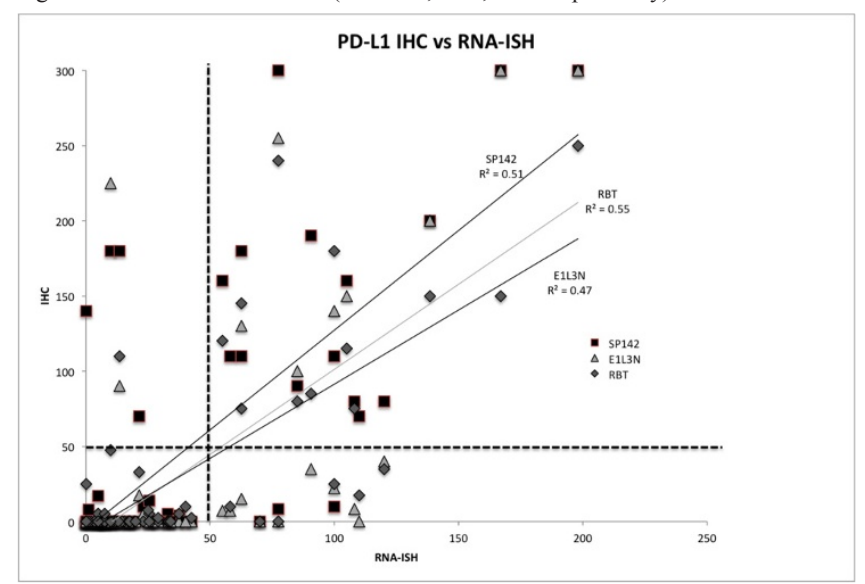

Conclusions: RNA expression correlates with protein expression for PD-L1. RNA ISH offers an attractive adjunct to IHC for PD-L1 assessment. This tool can help standardize laboratory assessment of PD-L1 expression. Using a dual IHC/ISH approach has the potential to offer robust and reproducible biomarker testing for both clinical trials and identification of eligible patients for PD-1 axis inhibitors.

1920 Reproducibility in Classification of Small Lung Adenocarcinomas: An International Interobserver Study

Angela R Shih, Alona Muzikansky, Emine Bozkurtlar, Jin-Haeng Chung, Yuko Minami, Lida P Hariri, Andre Moreira, Hironori Uruga, He Wang, Akihiko Yoshizawa, Mari Mino-Kenudson. Massachusetts General Hospital, Boston, MA; Marmara University, Istanbul, Turkey; Seoul National University, Bundang Hospital, Seongnam, Republic of Korea; National Hospital Organization, Ibarakihigashi National Hospital, Naka, Japan; Memorial Sloan Kettering Cancer Center, New York City, NY; Temple University School of Medicine, Philadelphia, PA; Kyoto University Hospital, Kyoto, Japan.

Background: The 2015 WHO classification for lung adenocarcinoma (ACA) provides criteria for diagnosis of adenocarcinoma in-situ (AIS), minimally invasive adenocarcinoma (MIA), and invasive adenocarcinoma (INV). Differentiating these entities can be difficult, and as understanding of prognostic significance increases, inconsistent classification is problematic. This study assesses agreement within an international panel of lung pathologists and identifies factors contributing to inconsistent implementation of the WHO criteria.

Design: Sixty cases of lung ACAs $(\leq 2 \mathrm{~cm})$ were reviewed by an international panel of 6 lung pathologists. One slide reflecting overall morphology of each case was digitally scanned to an internet browser-based viewer with a digital ruler. The panel independently reviewed each case to assess predominant pattern, invasive component size, and final diagnosis (AIS, MIA or INV). Statistical analysis was performed.

Results: The overall kappa value for AIS versus MIA and INV is 0.340 (fair agreement), and that for AIS and MIA versus INV is 0.438 (moderate agreement). The raters had $100 \%$ agreement on final diagnosis in 10 cases (AIS, $n=2 ;$ MIA, $n=2$; INV, $n=6$ ). In 28 cases with poor agreement on final diagnosis and invasive measurement (range: up to $13 \mathrm{~mm}$; SD: up to $5.4 \mathrm{~mm}$ ), inconsistent measurement of multifocal invasion led to wide variance in 5 cases and subjectivity in pattern recognition led to variance in 23 cases. Misinterpretation of the WHO criteria for MIA resulted in 18 instances of misclassification across all raters. A case with a predominant mucinous lepidic pattern had a range of diagnoses (AIS, $n=1 ;$ MIA, $n=1 ; I N V, n=4$ ).
Conclusions: Interobserver agreement on diagnosis of small lung ACAs between 6 raters was fair to moderate. Poor agreement cases were attributable to inconsistent measurement of multifocal invasion and subjectivity in pattern recognition. Misinterpretation of the WHO criteria contributed to inconsistent classification. Mucinous ACA may be more likely to be interpreted as invasive. A consensus conference with all raters will be held, followed by second and third rounds of evaluation to assess for improvement and the utility of ancillary tests (elastic stain).

1921 Discrepancy in Detection of Anaplastic Lymphoma Kinase (ALK) Gene Status in Lung Cancer by Immunohistochemistry (IHC), and Fluorescent In Situ Hybridization (FISH)

Hemlata Shirsat, Weei-Yuarn Huang, Wenda Greer, Mathieu Castonguay, Zhaolin Xu. Dalhousie University, Halifax, NS, Canada.

Background: Determination of anaplastic lymphoma kinase (ALK) gene status in lung cancer has become important in order to identify potential candidates for highly effective targeted chemotherapy. The gold standard test is fluorescent in situ hybridization (FISH). It is current practice in many centers using immunohistochemistry (IHC) as a screening test to examine ALK protein expression and use ALK FISH for confirmation of ALK gene rearrangement if IHC is positive or equivocal. Although the results of ALK IHC and FISH are consistent in general, the concordance is not $100 \%$ since the assays measure gene expression vs gene structure, respectively.

Design: We examined all ALK results including ALK IHC (5A4 clone) and ALK FISH for lung cancer in our file performed over the last 3 years and identified discrepancies between IHC and FISH in order to determine the level of concordance in detecting ALK abnormalities.

Results: A total of 98 cases were subjected to both FISH and IHC. Four of these cases demonstrated discordance between IHC and FISH. Three of these were positive for ALK by IHC but negative by FISH, and one case had negative result by IHC but was positive for ALK gene rearrangement by FISH. All cases equivocal by IHC were negative by FISH. One IHC positive/FISH negative case underwent further testing by RNA sequencing using next generation sequencing technology, which revealed an ALK gene rearrangement.

Conclusions: Discrepancy between IHC and FISH result is unusual but does exist. In such unusual circumstances, further verification using additional methods, such as next-generation sequencing, should be applied.

1922 Predictors of High Levels of Mutant cfDNA in Plasma from Patients with Advanced Lung Adenocarcinoma

Lynette M Sholl, Prodipto Pal, Ryan S Alden, Nora Feeney, Cloud Paweletz, Adrian Sacher, Geoffrey R Oxnard. Brigham and Women's Hospital, Boston, MA; Dana Farber Cancer Institute, Boston, MA; Belfer Center for Applied Cancer Science, Boston, MA. Background: Tumor mutation detection in plasma cell free DNA (cfDNA) is a promising approach to minimally invasive monitoring of patients with lung carcinoma. Prior studies have demonstrated that cfDNA genotyping correlates well with tumortissue based genotyping. However, individual tumors shed variable amounts of cfDNA into the circulation, with implications for applicability of cfDNA mutation monitoring in clinical practice. In this study, we examine the clinical, pathologic, and genomic features that correlate with high levels of mutant cfDNA in lung adenocarcinoma (ACA) patients with known tumor genotypes.

Design: The study includes patients with clinical stage IV lung ACA undergoing biopsy for newly diagnosed or progressive disease who also consented to blood collection for genomic analysis. Tumor histologic specimens were graded and subtyped according to WHO criteria by thoracic pathologists blinded to the results of the plasma genotype. For cfDNA detection, $10 \mathrm{~mL}$ of blood was collected in an EDTA-tube and plasma isolated via centrifugation within four hours. cfDNA was extracted from $2 \mathrm{~mL}$ of plasma using standard techniques. Droplet digital PCR was used for absolute quantification of $E G F R$ exon 19 deletion, L858R and T790M mutations, as well as KRAS codons 12-13 and $B R A F$ V600E mutations.

Results: A total of 70 patients with detectable mutations in cfDNA were evaluated including 29 at the time of new diagnosis and 41 with previously treated progressive disease, and included $49(70 \%) E G F R, 18(26 \%) K R A S$, and $2(3 \%) B R A F$ mutant tumors. Absolute cfDNA quantities ranged from 14 to 116,028 mutant copies $/ \mathrm{mL}$ of plasma (median 594 events); patients with cfDNA copies >median were considered high shedders. Tumor histologic features, including grade, histologic subtype, or presence of discohesive or infiltrating single cells did not correlate with levels of mutant shedding. High mutant shedding was significantly more common in patients with relapsed disease ( $61 \%$ versus $29 \%$ at new diagnosis, $\mathrm{p}=0.003$ ) and in $E G F R$ mutant tumors, but only in the relapse setting ( $67 \%$ of $E G F R$ versus $22 \%$ of KRAS/BRAF patients, $\mathrm{p}=0.006)$. EGFR T790M mutations were identified in $72 \%$ of high shedders versus $43 \%$ of low shedders at relapse (not significant; $\mathrm{p}=0.2$ ).

Conclusions: A high level of plasma cfDNA is more common in stage IV lung ACA patients at the time of progressive disease and correlates with tumor EGFR mutation but not tumor histology.

1923 Airway Centered Neutrophils in Post-Transplant Transbronchial Biopsies: A Poor Predictor of Infection

Payal Sojitra, Akshay Muralidhar, Sana Quddus, Swati Mehrotra, Vijayalakshmi Ananthanarayanan. Loyola University Medical Center, Maywood, IL.

Background: In post lung transplant surveillance settings, infections and rejection represent differentials for patients presenting with symptoms or drop in spirometry. Differentiating these entities is generally possible on morphology grounds. Neutrophils in the airway or within alveolar spaces is commonly interpreted in surgical pathology parlance as suggestive of an infectious process. In this study, we aim to evaluate the 
correlation between airway centered neutrophils in post-transplant transbronchial biopsies (PTBB) and concurrent bronchoalveolar lavage (BAL) culture results and cell counts.

Design: Pathology database (2010-2011) was gleaned for PTBB with the following search terms: "suspicious for infection" or "airway centered acute rejection". Patient demographics with pertinent clinical and laboratory data were obtained. Cases were graded as per ISHLT 2004 grading system. Histologic sections were also assessed for presence of neutrophils and highest number of neutrophils/HPF was calculated. We also looked at BAL neutrophilia, defined as $>4 \%$. Concurrent viral PCR results were not available on these cases.

Results: A total of 26 TBB obtained from 22 patients ( 8 females, 14 males) matched our criteria with a mean age of 52 years (range 28-72 years). The indications for transplantion included bronchiolitis obliterans $(n=1)$; COPD $(n=7)$; interstitial fibrosis $(n=8)$; cystic fibrosis $(n=6)$. Indications for TBB included: suspected infection $(n=10)$; drop in spirometry $(n=6)$ and surveillance $(n=10)$. Neutrophils within airways ranged from 1/HPF to 17/ HPF (mean 4.6/HPF). Measure of association between BAL culture results and neutrophils in the airway $\left(\right.$ Pearson $\mathrm{Chi}^{2}, \mathrm{p}=0.23$ ) is shown in table 1 .

\begin{tabular}{|l|l|l|}
\hline & \multicolumn{2}{|l|}{ Airway Centered Neutrophils } \\
\hline BAL Cultures & Present $(\mathrm{n}=15)$ & Absent $(\mathrm{n}=11)$ \\
\hline Positive $(\mathrm{n}=13)$ & 9 & 4 \\
\hline Negative $(\mathrm{n}=13)$ & 6 & 7 \\
\hline
\end{tabular}

BAL fluid neutrophil counts ranged from $0 \%$ to $93 \%$ (mean $31.8 \%$ ) with no correlation to culture results (Pearson $\mathrm{Chi}^{2}, \mathrm{p}=0.86$ ). Neutrophils within airways also did not correlate with either B component of acute cellular rejection or BAL neutrophilia. GMS and CMV stains were negative in all cases.

Conclusions: In our limited study set, we demonstrate that airway centered neutrophils in PTBB are poor predictors of culture positive BAL results. Alternative causes of acute airway inflammation including viral infections, airway stenosis, humoral rejection or aspiration need to be considered.

\section{Differential Expression of SOX17 in Lung and Endometrial} Carcinomas

Tracy A Stein, Mohamed El Hag, Bo Ye, Prachi Raut, Guang-Qian Xiao, Haodong Xu, Mahmoud Khalifa, Faqian Li. University of Minnesota Medical Center, Minneapolis, MN; University of Rochester Medical Center, Rochester, NY; UCLA Medical Center, Los Angeles, CA.

Background: Endometrial adenocarcinoma (EAC) metastatic to the lung poses diagnostic challenges, particularly when the primary tumor is not known. The use of TTF-1 can potentially be misleading as it can be positive in up to $19 \%$ of endometrial carcinomas. Although the use of PAX8 to evaluate metastatic Müllerian carcinomas is well established, PAX8 is also positive in thyroid and renal cell carcinomas. Therefore, there is utility in identifying additional markers for metastatic endometrial cancer. Sry-related HMG box transcription factor 17 (SOX17) plays an important role in embryogenesis and development, and we have previously shown its expression in EAC. The purpose of this study is to evaluate SOX17 expression in lung cancer to determine whether it can be used as a diagnostic marker to differentiate primary lung adenocarcinoma from metastatic EAC

Design: TMAs including 129 primary lung adenocarcinoma cases, 98 primary lung squamous cell carcinoma cases, and 36 cases of primary EAC were stained for SOX17. Subsequently, whole sections of 11 primary EAC cases and 10 metastatic EAC cases, including 4 metastatic to the lung, were stained for SOX17. A case was considered to be SOX17 positive if more than $10 \%$ of tumor cells showed moderate nuclear expression. Results: All lung adenocarcinomas and squamous cell carcinomas were distinctly negative for SOX17. In contrast, $72 \%$ (26/36) of EAC TMA cases were SOX17 positive. $100 \%(11 / 11)$ of primary EAC whole section cases showed weak to moderate nuclear SOX 17 positivity in at least $30 \%$ of tumor cells. All 10 metastatic EAC whole section cases showed moderate to strong nuclear SOX 17 positivity in at least $50 \%$ of tumor cells. Overall, metastatic EAC had stronger staining and more SOX17 positive tumor cells than primary EAC.

Conclusions: In this pilot study, SOX17 has been shown to be expressed in both primary and metastatic EAC, but distinctly absent in primary lung adenocarcinoma and squamous cell carcinoma. Therefore, SOX17 is a useful diagnostic marker to differentiate metastatic EAC from primary lung carcinoma. Further study of its diagnostic utility in a larger series and as a member of an IHC panel including PAX8 and ER is underway.

1925 Usefullness of p16 Immunostain and Molecular Testing for HPV in Determining Primary Versus Metastatic Squamous Cell Carcinoma in the Lung

Jamie Steinmetz, Brett E Close, Larissa V Furtado, Aliya N Husain, Nicole A Cipriani. University of Chicago, Chicago, IL.

Background: Primary lung squamous cell carcinoma (PLSCC) and metastatic squamous cell carcinoma (MSCC) to the lung can be morphologically indistinguishable, especially in the absence of a pulmonary in-situ component or central scar. A common clinical question is whether, in patients with primary head and neck carcinoma (HNSCC) or cervical SCC, a pulmonary SCC represents a metastasis or a second primary. If a primary HN or cervical SCC is HPV-associated, HPV status of the lung tumor could be useful. The aim of this study was to determine the rates of positivity of p16 IHC and presence of HPV DNA in PLSCC, in order to determine if p16/HPV status could be useful as an indicator of metastasis in the proper clinical context.

Design: 23 PLSCC cases were identified in patients with no known history of $\mathrm{HN}$ or cervical carcinoma. A tissue microarray of these cases was stained with p40 and p16 immunostains. p40 was considered positive if diffuse nuclear staining was present.
Any p16 staining was noted. Multiplex qPCR assay (which detects HPV subtypes 16, $18,31,33,45,52$ and 58 ) was performed on all available cases with any p16 staining. Additionally, our records were searched for lung SCC cases in which there was a known HPV-associated HN or cervical primary.

Results: All 23 PLSCC cases showed p40 positivity. 35\% (8/23) PLSCCs showed some staining with p16 (4 cases with moderate-strong nuclear and cytoplasmic staining in $>80 \%$ of tumor cells, 2 with weak-moderate nuclear and cytoplasmic staining in $60 \%$, 2 with only cytoplasmic staining ( 1 weak $<50 \%, 1$ moderate $70 \%)$ ). Multiplex qPCR assay was performed on the 7 available cases with p16 staining and all were negative. Record search revealed two cases of lung SCC in patients with prior HPV-associated HNSCC or cervical SCC: one tonsillar SCC with lung metastasis, both of which were positive for p16 and HPV16 DNA; one uterine cervical SCC with lung metastasis, in which the lung SCC was positive for p16 and HPV45 DNA. The primary cervical tumor was not available for testing.

Conclusions: We conclude that staining for p16 alone in SCC in the lung should not be used as an indicator of the presence of HPV DNA, and is not helpful in distinguishing PLSCC from MSCC. p16 IHC may be helpful when a HN or cervical $\mathrm{SCC}$ is p16 positive and the lung tumor is p16 negative. In the context of a HN or cervical SCC, molecular testing showing the same HPV DNA in the primary and lung tumor is the best evidence for metastasis.

1926 Elevated Integrin $\alpha 6 \beta 4$ Expression Is Associated with Venous Invasion and Decreased Survival in Squamous Cell Lung Cancer Rachel Stewart, Dava West, Chi Wang, Heidi Weiss, Tamas Gal, Eric Durbin, William O'Connor, Min Chen, Kathleen L O'Connor. University of Kentucky, Lexington, KY. Background: Lung cancer carries a poor prognosis and is the most common cause of cancer-related death worldwide. The integrin $\alpha 6 \beta 4$ is a laminin receptor that has been implicated in promoting carcinoma progression by cooperating with growth factor receptors and amplifying downstream signaling pathways such as PI3K, AKT, and MAPK. In carcinoma cells with mutant TP53, the integrin $\alpha 6 \beta 4$ promotes cell survival. TP53 mutations and integrin $\alpha 6 \beta 4$ overexpression co-occur in many aggressive malignancies. Given that lung squamous cell carcinoma is an aggressive malignancy with a high frequency of TP53 mutations, we predicted that integrin $\alpha 6 \beta 4$ expression in this tumor type would associate with poor prognosis.

Design: In order to investigate integrin $\alpha 6 \beta 4$ expression as it relates to clinicopathologic features and patient survival in non-small cell lung cancer (NSCLC), we constructed a lung cancer tissue microarray (TMA) $(N=211)$. TMA sections were stained for expression of the integrin $\beta 4$ subunit using immunohistochemistry. In addition, we analyzed multiple external gene expression datasets (TCGA, Hou) for integrin $\beta 4$ expression.

Results: Using our TMA data, we found that integrin $\beta 4$ expression is elevated in squamous cell carcinomas when compared to adenocarcinomas $(P<0.0001)$, which was confirmed in external gene expression datasets $(P<0.0001)$. We also found that integrin $\beta 4$ overexpression is associated with the presence of venous invasion $(P=0.0032)$, and with reduced overall survival (Hazard ratio $1.457,95 \%$ confidence interval 1.013 to 2.094, $P=0.0422$ ) among patients with NSCLC. Integrin $\beta 4$ mRNA overexpression was also shown to associate with reduced overall survival in a TCGA NSCLC dataset (Hazard ratio $1.714,95 \%$ confidence interval 1.052 to $2.792, P=0.0305$ ). Using cBioPortal, we generated a network map demonstrating the 50 most highly altered genes neighboring ITGB4 in squamous cell lung cancer. These genes included various laminins, collagens, CD151, EGFR, PIK3CA, MET, and YES1.

Conclusions: In conclusion, we demonstrate that integrin $\beta 4$ is overexpressed in lung $\mathrm{SCC}$, and that it is an adverse prognostic marker.

1927 Comprehensive Genomic Profiling of Lung Cancer is Practical and Facilitates Implementation of the National Comprehensive Cancer Network (NCCN) Guidelines for Lung Cancer Biomarker Testing in Patients with Advanced Stage Disease

James Suh, Adrienne Johnson, Kai Wang, Garrett Frampton, Juliann Chmielecki, Julia A Elvin, Jo-Anne Vergilio, Siraj M Ali, Vincent A Miller, Philip J Stephens, Jeffrey S Ross. Foundation Medicine, Inc., Cambridge, MA; Albany Medical College, Albany, NY. Background: The NCCN guidelines for lung cancer patients with metastatic disease recommend that $E G F R$ mutation and $A L K$ rearrangement studies should be performed as part of a multiplex or next-generation sequencing (NGS) panel that also includes testing for BRAF and ERBB2 mutations, MET amplification and ROS1 and RET rearrangements. Comprehensive genomic profiling (CGP) is a hybrid capture-based NGS test that can detect genomic alterations (GA) in hundreds of cancer-related genes efficiently, rapidly and accurately. Our aim is to investigate the feasibility and utility of CGP in clinical practice.

Design: DNA was extracted from 40 microns of FFPE sections from 7493 consecutive cases of lung adenocarcinoma, adenosquamous carcinoma, NSCLC-NOS and large cell carcinoma (2013-15). CGP was performed using a hybrid-capture, adaptor ligation based NGS assay to a mean coverage depth of $572 X$. GA (point mutations, small indels, copy number changes and rearrangements) involving EGFR, $A L K, B R A F, E R B B 2, M E T$, $R O S 1, R E T$ and KRAS were recorded for each case. In addition to patient age, sex and histologic type, the tissue sample site was reviewed for each case.

Results: The median age was 64 years (range 14-88) and 58\% were female. In this series, 5928 (79\%) lung adenocarcinomas, 85 (1\%) adenosquamous carcinomas, 1440 $(19 \%)$ NSCLC-NOS and 40 large cell carcinomas $(0.5 \%)$ were tested by CGP. $47 \%$ of specimens were obtained from extra-pulmonary metastatic sites. Overall, $5346(71 \%)$ patients harbored at least one GA involving EGFR (20\%), ALK (4.0\%), BRAF (5.7\%), $\operatorname{ERBB2}(5.7 \%), \operatorname{MET}(5.2 \%), \operatorname{ROS1}(1.5 \%), \operatorname{RET}(2.4 \%)$ and $K R A S(32 \%)$. A minority of patients harbored multiple GA in one or more of these genes, likely attributable to resistance mutations. The difference in the numbers of adenocarcinomas $(75 \%)$ and 
adenosquamous carcinomas $(69 \%)$ with at least one of the GA listed above versus NSCLC-NOS (58\%) and large cell carcinoma (42\%) was statistically significant (p-value $<0.0001)$.

Conclusions: CGP is practical and facilitates implementation of the NCCN guidelines for lung cancer biomarker testing by enabling efficient, rapid and accurate detection of GA involving all seven driver oncogenes listed by NCCN and KRAS. Furthermore, this study reinforces tissue-based NGS testing as the gold standard to which other types of assays can be compared for evaluation of sensitivity and specificity.

1928 Janus Kinase 3 (JAK3) Aberrations in Non-Small Cell Lung Cancer Laura N Toth, Christopher I Amos, Konstantin H Dragnev, Gregory J Tsongalis, Laura $J$ Tafe. Dartmouth-Hitchcock Medical Center, Lebanon, NH; Norris Cotton Cancer Center, Lebanon, $\mathrm{NH}$.

Background: Janus kinase $3(J A K 3)$ is a cytoplasmic tyrosine kinase expressed in cells of hematopoietic origin and mediates response to certain cytokines by activation of the STAT pathway. JAK3 mutations are associated with the development of some types of leukemia and have been reported at a low frequency in solid tumors. A recent report suggests that JAK3 activation in lung cancer cells induces PD-L1 expression, an important target of immunotherapy. In this study, we examine the prevalence and features of non-small cell lung cancers (NSCLC) with JAK3 mutations.

Design: In May 2013 our laboratory began using the Ion Torrent PGM and the AmpliSeq Cancer Hotspot Panel v2 for clinical practice. Briefly, DNA was extracted from unstained FFPE slides with a minimum tumor cellularity of $10 \%$. DNA was quantified using the PicoGreen method. Barcoded libraries were prepared using up to 10ng of DNA; sequencing was performed on the Ion Torrent PGM (318 chip). Variants were identified using the Ion Torrent Variant Caller Plugin and reference genome hg19. Golden Helix's SVS software was used for annotation and prediction of the significance of the variants. Tumor tissue was also tested for PD-L1 expression by immunohistochemistry (IHC) using PD-L1/CD274 monoclonal antibody (clone SP142) (Bioscience)

Results: Out of 288 NSCLC cases successfully sequenced between May 2013 December 2014, 8 (2.8\%) harbored a $J A K 3$ c.2164G $>A$ (p.V722I) mutation. The patients had a median age of 67.5 years and six of the patients were female $(75 \%)$. Five were current smokers, 2 former smokers, and 1 a non-smoker with significant second-hand exposure. Five patients presented with stage IV disease with CNS metastases; 4 are deceased, 1 is lost to follow up. In 5 instances $(63 \%)$, the tumors also had a KRAS mutation (p.G12C in 4, p.G12V in 1). Additional mutations were identified in $K I T(1)$, MET (1), PDGFRA (1), STK11 (2), andTP53 (3). Tissue was available for IHC in 6 cases, only two of which showed PD-L1 expression in $>5 \%$ of tumor cells.

Conclusions: Recurrent $J A K 3$ p.V772I mutations are found in a subset of NSCLCs, often associated with known smoking related KRAS mutations. The mutations occur in exon 15 which encodes for the JH2 pseudokinase domain of JAK3. Additional studies are needed to understand the functional effect of $J A K 3$ mutations in the development of NSCLC and the potential implications for immunotherapy or JAK3 inhibitors.

1929 Histologic Patterns of Resected Lung Adenocarcinoma Brain Metastases and Correlation with Molecular Analysis

Laura N Toth, Laura J Tafe. Dartmouth-Hitchcock Medical Center, Lebanon, NH. Background: Metastatic disease to the brain is common in patients with advance stage lung non-small cell carcinoma. Treatment of brain metastases may include chemoradiation, stereotactic ablative radiosurgery or tumor resection. To our knowledge, there have been limited studies that examine the histologic patterns of resected lung adenocarcinomas (ADC) and correlate with molecular analysis.

Design: We examined 29 consecutive cases of surgically resected brain metastases from 2012-2014 and classified the morphology according to the 2015 WHO classification while also including the recently recognized cribriform pattern of acinar which is associated with poor prognosis. Molecular genotyping had previously been performed either by single gene assays for EGFR, KRAS and BRAF (prior to 2013) or by next generations sequencing (NGS) with the 50 gene AmpliSeq Cancer Hotspot Panel v2 (Ion Torrent). This testing was done on the brain metastasis (23), lung primary (4) or a lymph node metastasis (2). Histology was reviewed without knowledge of mutation status. Results: Of the 29 cases, the predominant histologic variant was the solid type (59\%, 17 cases) followed by papillary (14\%, 4 cases) and cribriform (14\%, 4 cases). Less commonly appreciated were the micropapillary ( 2 cases), and one case each of acinar, and colloid patterns. Mutations in TP53 and KRAS were the most frequently identified (13 and 11, respectively). Seven of the KRAS mutations were seen in tumors with solid pattern. Interestingly, none of the cases with cribriform or micropapillary patterns harbored KRAS mutations, however, this was only 6 cases. NGS testing identified clinically important mutations that would have gone undetected with single gene analysis including an EGFR ex 20 insertion, ERBB2 ex 20 insertion and two PIK3CA mutations. Conclusions: Perhaps not surprisingly, in this patient cohort who underwent surgical resection of brain metastases, the majority of tumors showed what are considered aggressive morphologic patterns (solid, cribriform and micropapillary; $\mathrm{N}=21$ ) and frequent KRAS mutations. The next phase of this study will compare the metastatic tumors to the primary lung cancers when available and correlate with patient characteristic, treatment and outcome. Understanding the histopathology of lung $\mathrm{ADC}$ brain metastases can potentially help guide treatment and perhaps contribute to, ultimately, the prevention of brain metastases.
1930 Histologic Findings of Video-Assisted Thoracoscopic Surgery Lung Biopsies Performed in Patients with Treatment-Resistant Severe Asthma

Humberto Trejo Bittar, Daniel Doberer, Mitra Mehrad, Sally Wenzel, Samuel A Yousem. University of Pittsburgh Medical Center, Pittsburgh, PA.

Background: Severe asthmatic patients suffer from a disease that is poorly understood and uncontrolled with traditional therapy. The histologic changes occurring in ATS/ERS defined "severe asthma", particularly at the level of the distal airways remain largely unknown. We aimed to describe the morphologic changes in the distal lung in these severe asthmatics studied by video-assisted thoracoscopic surgery (VATS) lung biopsies. Design: 43 VATS biopsy specimens from "treatment-resistant severe asthma" (TRSA) patients were available for histologic examination. Histologic variables studied included goblet cell metaplasia, basement membrane thickness, airway inflammatory infiltrates and muscle thickness, alveolar septal infiltrates and presence and location of granulomas. Final pathological observations were correlated with clinical presentation and serological data.

Results: Of the 43 "TRSA" cases, 4 (9\%) showed non-asthma related pathology (3 hypersensitivity pneumonitis (HSP), 1 chronic aspiration). The remaining 39 cases demonstrated conventional histologic findings of asthma often with intense lymphocytic bronchiolitis (LB) $(16 / 39=41 \%)$, asthmatic granulomatosis (AG) $(20 / 39=51 \%)$, and non-diagnostic airway changes $(3 / 39=8 \%)$. Unexpectedly, 22 of $39(56 \%)$ of TRSA patients had non-disease defining autoantibodies or a family history of autoimmune disease (AID), among which $13(59 \%)$ presented with AG; whereas in those without history of AID, only $41 \%$ (7/17) had evidence of AG.

Conclusions: Histologic examination of VATS lung biopsies in "TRSA" demonstrates diverse pathologic findings including other diagnoses (HSP and aspiration). AG was present in $51 \%$ of TRSA patients; therefore pathologists should be aware of this new pathologic entity. Importantly, history of AID was present in $56 \%$ of TRSA patients, of which $59 \%$ (13/22) were diagnosed as AG. Our data suggest that histologic findings in TRSA patients, might represent a hybrid of asthma and lung involvement by AID; therefore, VATS lung biopsies should be considered to identify patients who will benefit from treatment options beyond conventional corticosteroids.

1931 Expression of Programmed Death-Ligand 1 in Small Cell Lung Cancer May Correlate with Better Patient Outcome: Analysis of 29 Cases Pamela Villalobos, Kathryn A Gold, Edwin Parra, Barbara Mino, Jaime RodriguezCanales, Reza J Mehran, Cesar A Moran, Ignacio I Wistuba, Bonnie Glisson, Neda Kalhor. MD Anderson Cancer Center, Houston, TX.

Background: Key elements in promoting the immunosuppressive environment of tumor cells include upregulation of inhibitory pathways such as programmed death-ligand 1/programmed death1 (PDL-1/PD-1). PD-L1 expression and its clinical relevance in small cell lung cancer (SCLC) are not fully understood. We investigated the correlation between immunohistochemical (IHC) expression of PD-L1 in SCLC and the expression of PD1 in its tumor-infiltrating lymphocytes (TILs) population, and correlated those profiles with clinico-pathological data including patients' outcome.

Design: A total of 29 resected SCLCs were collected retrospectively from the archives of the Department of Pathology at MD Anderson Cancer Center. PD-L1 expression and density of TILs expressing PD-1 and CD3 were evaluated by immunohistochemistry (IHC) and digital image analysis (Aperio) in the intratumoral compartment. From the total tumor region, 5 random $1 \mathrm{~mm}^{2}$ areas were selected for PD-L1 expression analysis. PD-L1 was evaluated as percentage of tumor cells showing membrane positivity, and TILS were quantified as cell density (number of cells $/ \mathrm{mm}^{2}$ ).

Results: $69 \%$ of SCLC cases (20 of 29 ) showed $>1 \%$ PD-L1 positivity within tumor cells. PD-L1 expression showed significant correlation with TILs expressing PD-1 $(\mathrm{r}=0.797, P<0.001)$ and $\mathrm{CD} 3(\mathrm{r}=0.759, P<0.001)$. Patients' outcome analysis using Kaplan-Meier showed that PD-L1 expression ( $>1 \%$ of positive cells) correlated with better overall survival (OS; $P=0.001$, univariate analysis). PD-L1 expression using others different cutoff $(5 \%, 10 \%, 20 \%)$ did not have any significant correlation with OS. Conclusions: Our data shows that majority of SCLC have $>1 \%$ positive PD-L1 expression, by immunohistochemical analysis and contain $\mathrm{PD}-1$ positive

T-cells. Further study of PD-L1 as a prognostic factor and potential target for therapy is warranted.

1932 "Borderline" Neuroendocrine Carcinomas of the Lung Are Clinically and Genomically Distinct from Large Cell Neuroendocrine Carcinoma

Marina Vivero, Lynette M Sholl. Brigham and Women's Hospital, Boston, MA. Background: Small cell lung carcinoma (SCLC) is characterized by brisk tumor proliferation, high mutational frequency, and RB1 and TP53 loss of function, while typical (TC) and atypical carcinoids (AC) show low proliferation and lack mutations in these genes. Pulmonary large cell neuroendocrine carcinoma (LCNEC) has a high proliferation rate $(>10$ mitoses $/ \mathrm{mm} 2)$, but a better prognosis and more heterogeneous molecular profile than SCLC. A subset of tumors that resemble AC but have $>10$ mitoses/ $\mathrm{mm} 2$ are classified as LCNEC per the World Health Organization (WHO); however, evidence supporting this classification is lacking. We examine the clinical and genomic features of these "borderline" LCNEC (BT).

Design: Included were TC and AC (grouped hereafter as low grade tumors, LG);BT; and LCNEC and SCLC (grouped as high grade tumors, HG) resected between 2013 and 2015 and classified according to WHO 2015 criteria. Targeted hybrid capture next generation sequencing for 309 cancer-related genes was performed for detection of single nucleotide variants (SNVs), copy number variants (CNV) and rearrangements. Results: 59 tumors including 9 TC, 7 AC, 7 BT, 11 LCNEC, and 25 SCLC were obtained from 31 women and 27 men. Patients with LG and BT were younger (56.8 and 56.4 vs 
65.6 years, $\mathrm{p}=0.01)$, less frequently smokers $(9,56 \%$ and $2,29 \%$ vs. $33,94 \%$; $\mathrm{p}=0.001)$, and smoked fewer pack-years ( 8.9 and 8.3 vs $50.4, \mathrm{p}<0.0001)$ than patients with HG. BT and HG had higher numbers of SNVs compared to LG, (3.1 vs 6.1 vs 9.7 in LG, $\mathrm{BT}$, and HG, $\mathrm{p}<0.0001)$, but BT and LG had significantly fewer gene-level CNVs than HG (29.1 vs 38 vs 68 in LG, BT, and HG, p=0.0013). 9 (82\%) LCNEC and $23(92 \%)$ SCLC had loss-of-function alterations in TP53 and/or $R B 1$, while no TC, AC, or BT had these alterations. Other mechanisms of cell cycle dysregulation were seen in BT including CDKN2A deletion $(n=1)$ and CCND1 amplification $(n=1)$. Oncogenic alterations typical of lung adenocarcinoma were detected in 4 LCNEC (EGFR, KRAS, MET), one BT (AKT1+BRAF), one SCLC (EGFR), and no TC or AC. MEN1 mutations were seen in $2(28 \%)$ AC, $1(14 \%)$ BT and $1(9 \%)$ LCNEC.

Conclusions: Borderline tumors show a diverse profile of oncogene and tumor suppressor gene alterations. They are distinct from LCNEC in their younger age of onset, lack of a significant smoking association, absence of TP53 and RB1 alterations, and less pronounced copy number instability. These findings may have significant implications for selection of therapy and should be taken into consideration in pathologic classification.

\section{HPV Prevalence and Genotype in Squamous Cell Carcinoma of the Lung}

Christopher Vytlacil, Christina DiCarlo, Emily J Smith, Matthew A VanDeusen, Samantha A Martin, Lori A Kelly, Ali Zaidi, Gene Finley, Rodney J Landreneau, Blair A Jobe, Jan F Silverman. Allegheny General Hospital, Pittsburgh, PA; Allegheny Health Network, Pittsburgh, PA

Background: Human papilloma virus (HPV) has been reported as a potential cause in some cases of lung cancer. A few previous studies report from $6-24 \%$ of non-small cell carcinomas to be positive for HPV with high risk HPV 16 and 18 genotypes. We performed molecular studies to determine the prevalence of HPV positivity in squamous cell carcinomas of the lung and to determine the most common genotypes.

Design: Pulmonary squamous cell carcinomas and HPV positive cervical formalinfixed paraffin-embedded (FFPE) tissue samples with corresponding H\&E slides were analyzed. All cases had $20 \mu \mathrm{m}$ sections prepared and mounted onto microscopic slides. They were then macrodissected to remove the tumor area that was marked on the corresponding H\&E slides. The sections of tumor were stored in microcentrifuge tubes in a $-20^{\circ} \mathrm{C}$ freezer until DNA extraction could be competed. The Qiagen QIAamp DNA FFPE Tissue Kit was used to extract and purify the tumor DNA. The DNA was then quantitated using a spectrophotometer in preparation for PCR amplification using the INNO-LiPA HPV Genotyping Extra AMP Kit. After PCR amplification, the biotinylated lung DNA samples were tested for HPV using the INNO-LiPA HPV Genotyping Kit. One HPV positive cervical sample was used as a second positive control for each run in addition to the kit positive control. Water was utilized as a negative control in each run to ensure that there was no cross-contamination from HPV positive DNA. This kit is capable of identifying 32 individual HPV genotypes.

Results: One squamous cell carcinoma lung sample out of 68 tested positive for HPV. The lung sample was a $4.0 \mathrm{~cm}$ chemonaive stage IB cancer which tested positive for HPV genotype 11 , a low risk genotype, establishing a study prevalence of $1.5 \%$ in squamous cell carcinoma.

Conclusions: The prevalence of HPV positivity in squamous cell carcinoma of the lung is much lower in our series $(1.5 \%)$ than previously reported. Based on the study results, we believe that HPV DNA is uncommonly associated with squamous cell carcinoma of the lung.

1934 PD-L1, PD-1, CD4 and CD8 Expression in Neoplastic and NonNeoplastic Thymus

Ann E Walts, Alberto M Marchevsky. Cedars-Sinai Medical Center, Los Angeles, CA. Background: The ability of tumor cells to express PD-L1, programmed cell death protein-1, which binds with its receptor PD-1 on activated T-cells helps tumors avoid detection and allows for tumor growth and/or metastases. There is only limited information regarding the expression of PD-L1 and PD-1 in thymic epithelial tumors (TET).

Design: Serial sections from 38 thymomas (WHO type A ( $n=2), A B(n=9), B 1(n=9)$, B2 $(n=8), B 3(n=10), 8$ thymic squamous carcinomas (SqCA), and 20 non-neoplastic thymi (adjacent to the tumors $(n=16)$, hyperplastic $(n=2)$, and normal of childhood $(\mathrm{n}=2))$ were stained with antibodies against PD-L1 (clone SP142 Spring Bioscience), PD-1 (MRQ22 Cell Marque), CD4 (clone SPO32 Cell Marque), and CD8 (JCB117 Ventana). Membranous and/or cytoplasmic staining for PD-L1 and PD-1 in $\geq 5 \%$ of thymic epithelial cells, tumor cells or lymphocytes was scored as positive.

Results: PD-L1 was expressed in $55 \%$ of non-neoplastic thymi, $22 \%-100 \%$ of thymomas and $37.5 \%$ of SqCA. PD-L1 expression in TET was equal to or higher than that seen in adjacent non-neoplastic thymi in $75 \%$ of paired cases. A significantly higher $(\mathrm{p}<0.02)$ proportion of B2 $(100 \%)$ and B3 $(90 \%)$ thymomas were positive for PD-L1 than non-neoplastic thymi, $\mathrm{A}, \mathrm{AB}$ and $\mathrm{B} 1$ thymomas and SqCA. PD-1 was expressed in $10 \%-22.2 \%$ of cases. There was no significant difference $(\mathrm{p}>0.05)$ in the proportion of PD-1 positive cases by diagnosis, and PD-1 expression did not appear to correlate with PD-L1 expression within individual cases or across diagnostic groups. PD-L1 expression was diffuse and predominantly seen in epithelial cells while PD-1 expression was often observed in scattered and/or small groups of lymphocytes and/or in germinal centers. TET contained variable numbers of CD4+ and CD8+ lymphocytes, according to WHO type. CD4+ cells were generally concentrated at the periphery of neoplastic epithelial cells in B2 and B3 thymomas and SqCA; CD8+ lymphocytes were diffusely admixed with the epithelial tumor cells of these lesions.
Conclusions: PD-L1 expression is more frequent in B2 and B3 thymomas than in other TET, including SqCA, and non-neoplastic thymi. PD-L1 expression does not appear to correlate with PD-1, CD4 or CD8 expression, raising the possibility that the PD-L1/ PD-1 checkpoint pathway may not play a major role in TET.

1935 BAP1 Immunostain and P16 FISH: Clinical Applicability to Confirm Malignant Mesothelioma in Effusions

Ann E Walts, Kenzo Hiroshima, Stephanie McGregor, Aliya N Husain, Alberto M Marchevsky. Cedars-Sinai Medical Center, Los Angeles, CA; Tokyo Women's Medical University Yachiyo Medical Center, Yachiyo, Japan; University of Chicago Hospitals, Chicago, IL.

Background: Immunohistochemistry (IHC) helps distinguish carcinoma from malignant mesothelioma (MM), but not reactive mesothelial cells from MM. Loss of BAP1 expression by IHC and p16 deletion by fluorescence in-situ hybridization (FISH) have been proposed as adjuncts to diagnose MM in effusions but their clinical applicability in practice is still uncertain.

Design: Paraffin embedded cell block sections from 63 pleural and 4 peritoneal effusions from 32 patients with biopsy confirmed MM and 35 with reactive effusions were stained using a monoclonal antibody against BAP1 (clone 4, Santa Cruz Biotech.). 38 (56.7\%) of the 67 cell blocks ( $17 \mathrm{MM}$ and 21 reactive) were also analyzed for p16 deletion by FISH. Only mesothelial cells exhibiting atypical morphology were evaluated. Criteria for MM were: absence of nuclear staining for BAP1 in $>50 \%$ of the atypical mesothelial cells in the presence of a positive internal control, p16 homozygous deletion in $>15 \%$ or hemizygous deletion in $>41.5 \%$ of atypical mesothelial cells analyzed (Wu et al. AJCP 2013;139:39-46). Sensitivity (SS), specificity (SP), and positive and negative predictive values (PPV, NPV) for the diagnosis of MM were calculated for each test. Results: 17 of 32 MM were correctly diagnosed by BAP1 IHC. The SS, SP, PPV, NPV of BAP1 IHC for MM were $53.1 \%, 85.7 \%, 77.3 \%$, and $64.3 \%$, respectively. In the 38 cases studied by IHC and FISH, the SS, SP, PPV, and NPV of IHC for MM were $41.2 \%, 81.0 \%, 63.6 \%$, and $63.0 \%$, respectively. 7 of $17 \mathrm{MM}$ were diagnosed by FISH; 6 showed homozygous and 1 hemizygous deletion patterns. SS, SP, PPV, and NPV of FISH for MM in these 38 cases were $41.2 \%, 100 \%, 100 \%$, and $67.7 \%$, respectively. 10 of the 15 false negative cases by BAP1 IHC were also studied by FISH: 6 were also false negative by FISH and 4 were diagnosed correctly by FISH. 4 of the 5 false positive cases by BAP1 IHC were studied and correctly diagnosed by FISH. 2 MM correctly diagnosed by FISH were false negative by IHC.

Conclusions: BAP1 IHC and p16 FISH yield similar sensitivities for MM in paired samples but FISH has a higher specificity. As IHC is more readily available and easier to interpret in most laboratories, BAP1 IHC to screen effusions with atypical mesothelial cells and confirmation by FISH provides a cost effective algorithm for diagnosis of these cases. This strategy would reduce the number of cases requiring FISH analysis without significant differences in NPVs.

\section{Pure Atypical Thymomas: A Clinicopathological Analysis of 25 Cases}

Annikka Weissferdt, Maheshwari Ramineni, Neda Kalhor, Cesar A Moran. MD Anderson Cancer Center, Houston, TX.

Background: Thymomas are rare neoplasms of the anterior mediastinum. Atypical thymoma (AT)(WHO type B3) is an infrequent subtype of thymoma characterized by an epithelial-rich histology with limited immature T lymphocytes. Pure AT are uncommon as the majority of thymomas demonstrate mixed histology if sufficiently sampled. Lack of stratification by histologic type has prevented large scale research into these tumors and focused studies on this tumor type are lacking. We report the clinicopathological features of 25 such tumors, in one of the largest series of these tumors to date.

Design: Twenty-five cases of pure AT from the surgical pathology files of the MD Anderson Cancer Center were analyzed after review of 400 thymic epithelial neoplasms and after exclusion of cases with merely an AT component. An average of 10 tumor sections from thymectomy specimens was available for review in each case; cases with fewer than 5 sections were excluded from this study. Clinical information including presentation, treatment and follow up was obtained from the referring pathologists or institutional clinical files.

Results: The patients were 11 females and 14 males with an age range from 19 to 75 years (mean, 54). Main presenting symptoms included chest pain, shortness of breath and dyspnea. Myasthenia gravis was present in 7 patients. The average tumor size was $7.2 \mathrm{~cm}$. Histologically, the tumors were characterized by a lobulated epithelial proliferation with mild to moderate cytologic atypia, occasional perivascular spaces and scarce infiltrate of T cells. Using Moran's staging schema, 11 patients were stage I, 5 stage $2 \mathrm{a}, 6$ stage $2 \mathrm{~b}$ and 1 stage $3 \mathrm{a}$ and $3 \mathrm{~b}$, respectively. Sixteen patients received neoadjuvant and/or adjuvant chemoradiation. Clinical follow-up available for 22 patients showed that 15 were alive with a follow-up period from 5-140 months (mean, 54), 7 of which with stable recurrent and/or metastatic disease. Seven patients had died 24-172 months after diagnosis (mean, 87), 3 from unrelated causes.

Conclusions: Pure AT are tumors predominantly affecting patients in the $6^{\text {th }}$ decade of life with a slight male predilection. Although AT are widely regarded as an intermediate form between thymoma and thymic carcinoma, its clinical course is characterized by a low grade malignant behavior that is not much different from that of other types of thymoma. Although histology is important, the most relevant predictor of prognosis remains tumor stage at the time of diagnosis. The use of the Moran staging system appears to provide clear and direct pathological information with regard to the degree of invasiveness of the tumor. 
1937 Pleomorphic Carcinomas of the Lung: A Clinicopathological and Immunohistochemical Study of 68 Cases

Annikka Weissferdt, Maheshwari Ramineni, Neda Kalhor, Barbara Mino, Jaime Rodriguez Canales, Junya Fujimoto, Ignacio I Wistuba, Cesar A Moran. MD Anderson Cancer Center, Houston, TX.

Background: Pleomorphic carcinoma of the lung (PC) is a rare variant of lung cancer defined by the WHO as a poorly differentiated non-small cell lung carcinoma (NSCLC) that contains at least $10 \%$ giant and/or spindle cells or only giant and spindle cells. Their low incidence, lack of uniform diagnostic criteria and aggressive clinical course has impaired systematic research into these tumors. We report the clinicopathological and immunohistochemical features of $68 \mathrm{PC}$

Design: 68 pulmonary PC $(0.75 \%)$ were identified among 9000 resected NSCLC at our institution from 1986-2015. All cases were analyzed for clinicopathological parameters. In 56 cases, immunohistochemical studies with CAM5.2, CK5/6, p40, Sox2, desmocollin-3, CK7, TTF-1, napsin A, calretinin and D2-40 were performed. Results: The patients were 40 males and 28 females aged 36 to 87 years (mean, 64). Symptoms included chest pain, shortness of breath and hemoptysis. 57 patients had a smoking history. Histologically, the tumors were composed of malignant spindle and giant cells with or without differentiated NSCLC elements. 26 cases (28\%) contained differentiated elements (17 adenocarcinoma, 5 squamous cell carcinoma, 4 large cell carcinoma) by morphology and 42 cases $(62 \%)$ did not. Immunohistochemically, the pleomorphic component showed reactivity for CAM5.2 (86\%), p40 (4\%), CK7 (75\%), TTF-1 (41\%), napsin A (25\%), calretinin (18\%), Sox2 (13\%) and D2-40 (5\%). None of the cases expressed CK5/6 and desmocollin-3. By combination of morphological and immunohistochemical features, 33 cases (49\%) showed evidence of pneumocytic and 5 (7\%) of squamous differentiation. 41 patients received chemoradiation therapy. Clinical follow-up showed that 42 patients had died 1 to 88 months after diagnosis (mean survival 16.4); 26 patients were alive with a follow-up period from 1-145 months (mean 56.6). Conclusions: PC primarily affect patients in the $7^{\text {th }}$ decade with a M:F ratio of $1.4: 1$, have a strong association with smoking and an aggressive clinical course. Our results indicate that PC likely represent variants of poorly differentiated NSCLC. Thus, we recommend the use of immunohistochemistry in PC to determine any possible differentiation towards adenocarcinoma or squamous cell carcinoma. Such practice will lead to better identification of patients who may benefit from molecular testing and targeted therapies. A diagnosis of PC should be reserved for those cases which by a combination of morphology and immunohistochemistry show no distinct differentiation.

1938 Stromal Inflammation Score: A Prognostic H\&E Biomarker for Squamous Cell Carcinomas of the Lung

Daniel Xia, Ruben Casanova, Alex Soltermann, Andrew H Beck. Brigham and Women's Hospital, Boston, MA; University of Zurich, Zurich, Switzerland; Beth Israel Deaconess Medical Center, Boston, MA.

Background: Squamous cell carcinoma (SCC) is the second most common histologic subtype of non-small cell lung cancer. In comparison to lung adenocarcinomas, relatively less is known about the histologic determinants of survival in lung SCC. In a previous study, we performed a high-throughput computationally guided screen of lung SCC $\mathrm{H} \& \mathrm{E}$ histology, and identified cancer stroma chronic inflammation as a prognostic biomarker. Here, we report on additional efforts to validate this biomarker in univariate and multivariate survival analyses, and on efforts to better understand the molecular correlates of cancer stroma inflammation.

Design: We previously defined a stromal inflammation (SI) score for validation by human observers. Two observers, blinded to outcomes, scored all cases from a tissue microarray (TMA) image dataset. One observer, blinded to outcomes and molecular data, scored all full slide images available online through The Cancer Genome Atlas (TCGA) Network. Combining the scores with corresponding clinical and molecular data, we assessed for associations between the SI score and overall survival, somatic mutations, single nucleotide polymorphisms, and mRNASeq gene expression data. All statistical analyses were performed using $\mathrm{R}$ (version 3.2.2).

Results: The SI score is significantly associated with overall survival in both univariate and multivariate Cox proportional hazard (CPH) analyses in the TMA dataset. The effect size of the biomarker is substantial in multivariate $\mathrm{CPH}$ analyses (e.g. hazard ratio $=0.32,95 \% \mathrm{CI}=0.19-0.54, \mathrm{p}$-value $=2.2 \times 10^{-5}$ for one observer, see Table). The SI scores are also highly correlated between the two observers (Spearman rho $=0.88$ ). On molecular correlation studies using the TCGA dataset, the SI scores are positively correlated with the expression of genes involved in immune response.

\begin{tabular}{|l|l|l|}
\hline Variable & Hazard ratio $\mathbf{( 9 5 \%}$ CI) & P-value \\
\hline Overall stage & $1.58(1.36-1.84)$ & $3.1 \times 10^{-9}$ \\
\hline Age (years) & $1.04(1.02-1.05)$ & $4.7 \times 10^{-8}$ \\
\hline Vascular invasion & $1.81(1.42-2.32)$ & $2.1 \times 10^{-6}$ \\
\hline SI score & $\mathbf{0 . 3 2}(\mathbf{0 . 1 9 - 0 . 5 4 )}$ & $\mathbf{2 . 2 \times 1 0 ^ { - 5 }}$ \\
\hline Pleural invasion & $1.13(0.88-1.46)$ & 0.34 \\
\hline Tumor grade & $1.09(0.87-1.37)$ & 0.45 \\
\hline
\end{tabular}

Conclusions: Increased SI is associated with improved survival in lung SCC . The SI score requires only routine H\&E histology, a characteristic that should assist with further validation and research efforts.
1939 Simultaneous Detection of Gene Rearrangements and Variants in Non-Small Cell Lung Cancer by Next-Generation Sequencing

Shuo Xu, Alexander Boag, Mihaela Mates, Lina Chen, Harriet Feilotter. Queen's University, Kingston, ON, Canada.

Background: The treatment for non-small cell lung cancer (NSCLC) has been revolutionized by targeted therapy based on tumor mutation profiling. Currently, because different technologies are used to identify DNA variants and gene fusion events, serial single gene testing is the most common method used, which is expensive, tissue demanding and time consuming. Next generation sequencing (NGS) provides an avenue to examine both in a single assay. We used NGS to simultaneously interrogate clinically relevant DNA variants and gene fusion events, providing richer data about the tumours. Design: 21 consecutive formalin-fixed paraffin-embedded (FFPE) resected pulmonary adenocarcinomas underwent central pathology review and NGS. The assay, an AmpliSeq design using both DNA and RNA as input material, interrogated variants in approximately 50 cancer genes, as well as identifying fusion events that included ALK, ROS or RET. Gene rearrangement, single-nucleotide variants and small insertion or deletion events were reported, following filtering and alignment of the sequences using the Ion Torrent Suite and Ion Reporter software.

Results: Nineteen of the 21 samples had at least one mutation. KRAS was the most frequently mutated gene (12), followed by p53 (11), STK11 (4), MET (3), SMAD4 (3), RET (2) EGFR (2), SMAD4 (2), AKT1 (2), FGFR1 (1) and ERBB2 (1). The frequency of RET rearrangement was higher than expected (2). No ALK or ROS rearrangements were identified. 11 samples had more than one mutation, 7 of which had coexisting KRAS mutations. Both samples with RET rearrangement had coexisting mutations; one had concurrent KRAS mutation, contradictory to the current belief that RET rearrangements are mutually exclusive of KRAS mutation. Interestingly, KRAS mutation status appeared to correlate positively with survival, although this did not reach significance. Tumors with multiple mutations tended to have higher grade. To validate and extend these findings, we are continuing to accrue samples to strengthen the prognostic and predictive associations.

Conclusions: NGS is a tissue-conserving, time-efficient and versatile tool for molecular profiling of NSCLC, allowing simultaneous detection of translocation and targeted mutations using FFPE samples. The coexistence of multiple mutations is found to be a common event in our study, and these investigations will provide novel information about the co-occurrence and incidence of these rare variants, as well as their impact on clinical parameters.

1940 The Clinical Impact of Solid and/or Micropapillary Subtypes in Resected Lung Adenocarcinoma

Naoki Yanagawa. Yamagata Prefectural Central Hospital, Yamagata, Japan.

Background: Lung adenocarcinoma (ADC) presents various histopathologic images. Predominant growth patterns according to new ADC classification proposed by the International Association for the Study of Lung Cancer, American Thoracic Society, and European Respiratory Society (IASLC/ATS/ERS) are correlated with the outcome of the patients. In particular, the patients with solid or micropapillary predominant growth pattern show a worse prognosis than other predominant growth patterns. On the other hand, there are several cases which contain solid and/or micropapillary subtypes even if their subtypes do not present predominance. However the evaluation of these cases is not yet well determined. We aimed to investigate a clinical impact of these cases. Design: At first, we classified 531 ADCs according to the new ADC classification, and then, we reclassified the patients into 5 subgroups according to the proportion of solid (S) and/or micropapillary (MPP) subtype: 1) both subtypes absent (S-/MPP-), 2) solid predominant (S pre), 3) micropapillary predominant (MPP pre), 4) solid present although not predominant and micropapillary absent (S+ not pre/MPP-), 5) MPP present although not predominant (MPP+ not pre). The relationship between these results and clinicopathological data including outcomes was investigated statistically.

Results: Of the $531 \mathrm{ADCs}, 384$ were classified as S-/MPP- (72.3\%), 55 as S pre (10.4\%), 11 as MPP pre $(2.1 \%), 42$ as S+ not pre/MPP- $(7.9 \%)$, and 39 as MPP+ not pre (7.3\%). Recurrence-free survival (RFS) and overall survival (OS) differed among the 5 subgroups ( $\mathrm{p}<0.01$ and $\mathrm{p}<0.01$, respectively).

\section{Figure 1. Kaplan-Meier survival curve for recurrence free survival}

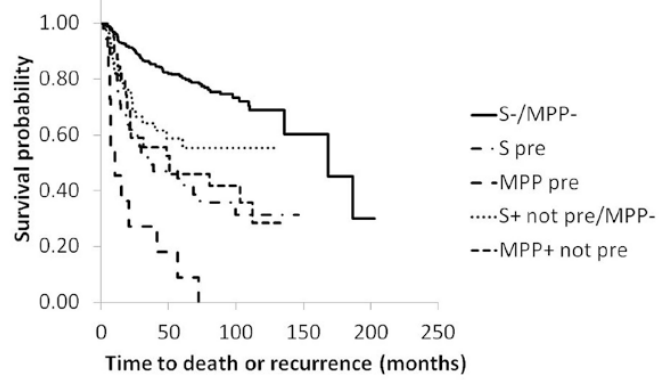

The S-/MPP- group showed a better RFS and OS than other groups. On the other hand, the MPP pre group showed a worse RFS and OS than S pre, S+ not pre/MPP- and MPP+ not pre groups. These three groups presented a similar survival curve of RFS and OS. Multivariate analysis revealed the recognization of S and/or MPP subtypes as independent predictors of RFS and OS ( $\mathrm{p}<0.01$, respectively). 
Conclusions: The patients with solid or MPP subtypes had a worse prognosis even if their subtypes were not predominant. Therefore we need to take care of solid or MPP subtypes at the time of diagnosis.

1941 Malignant Melanoma Presenting as Thoracic Midline Malignancy: Clinicopathological and Molecular Features

Keluo Yao, Weiqiang Zhao, David Cohen, Alejandro Gru, Greg Otterson, Konstantin Shilo. The Ohio State University Wexner Medical Center, Columbus, $\mathrm{OH}$.

Background: Malignant melanoma (MM) typically occurs in the skin, but also may occur in mucosal membranes and, rarely, visceral organs. Approximately $30-40 \%$ of cutaneous MM harbor activating BRAF V600 mutations providing the basis for treatment with BRAF kinase inhibitors. Primary thoracic MM is exremely rare with very few cases reported in the literature and their molecular profile is largely undefined. The aim of this study was to detail clinicopathological and molecular findings of MM in this uncommon location.

Design: Based on review of surgical pathology reports from January 2012 to June 2015, five cases of malignant melanoma presenting as a thoracic midline lesion were identified. The histological diagnosis was confirmed on subsequent re-review of H\&E and immunohistochemical stains. Sanger and/or Next Generation Sequencing were employed for molecular profiling. Review of electronic medical records revealed no evidence of an extrathoracic primary.

Results: The tumors occurred in 5 Caucasian patients ( 4 males and 1 female, medium age of 71.6 years, range from 54-80). The patients had site specific symptoms including dyspnea (2/5), cough (2/5), hemoptysis (1/5), and change in voice (1/5). At presentation, imaging studies showed disease confined to the chest (5 of 5), including middle mediastinum (4 of 5) and lung hilum (3/5). At light microscopy, 2 of 5 tumors featured cytoplasmic melanin. All five tumors showed expression of melanocytic markers. Four of 5 patients died of the disease within one year (mean follow up time five months). One tumor harbored BRAF V600K mutation and showed partial response to BRAF inhibitor. None of 4 tested cases showed BRAF V600E mutation.

Conclusions: MM presenting as a thoracic midline mass lesion elicits site specific symptoms likely due to central location associated with the injury to large airways. While their light microscopic morphology and immunohistochemical profile are identical to cutaneous MM, our analysis documents that MM in this location shows an aggressive clinical course and potentially different spectrum of BRAF mutations.

1942 Patchy High Frequency of Nonspecific Abnormal Signals (Background Noise): A Common Pitfall in Interpretation of ALK FISH Results

Hong Yin, Shaobo Zhu, Michele K Zelonis, Dana R Snyder, Zongming E Chen. Geisinger Medical Center, Danville, PA.

Background: The presence of EML4-ALK fusion as a consequence of ALK gene rearrangement is a useful biomarker to predict therapeutic responsiveness to anti-ALK agent in a subset of non-small cell lung cancer (NSCLC). The gold standard assay for detection of ALK rearrangement is by FISH analysis. Interpretation of FISH results can be difficult in some challenging cases. Patchy high frequency of abnormal signals is recognized as one potential pitfall that may lead to false positive results. Here, we report the frequency, distribution pattern, and useful remedy for this challenging diagnostic issue based on our own experience in Geisinger Medical Laboratory.

Design: A total of 188 FISH analysis for ALK gene rearrangement to investigate the background noise. In addition, there were 17 positive samples from our validation included in this study in order to compare the signal patterns. Cases with high background noise (abnormal signals $>10 \%$, falsely positive) were identified. FISH analysis was repeated using different specimens from the same patients. The signal patterns were carefully analyzed by two and more specially trained FISH technologists and pathologists. The characteristics of background noise signals were compared with true positive signals

Results: There were 3 of the $188(1.6 \%)$ cases with ALK gene rearrangement. A total of 20 ALK gene rearrangement samples were studied. In these 20 samples, the positive split signals showed a diffuse abnormal pattern. Non-tumor areas were compared, although abnormal signals can occasionally be seen, it is usually patchy and focal There are 5 cases that reveal high background noise, 3 cases up to $16-18 \%$ positive signals. The distribution of background noise appears as abnormal split signals were focal, and not a true diffuse pattern. When carefully examined abnormal signals were also found in adjacent non-tumor cells when compared to areas containing tumor. It would be interesting to confirm our hypothesis - background noise up to $18 \%$. For this study, we repeated ALK FISH assay for two patients using different specimens. Upon repeating with different specimens, the background noise decreased to 2-6\%. The third patient had tumor containing an EGFR mutation, essentially precluding any possible ALK gene arrangement.

Conclusions: Accurate assessment of ALK gene rearrangement by FISH is critical for identifying patients with NSCLC who may benefit from ALK inhibitor therapy. Our data indicate that it is important to recognize this background noise, which may be a potential pitfall in interpretation of ALK FISH results.

1943 Dysregulated miR-185-STIM1 Axis in Obliterative Bronchiolitis Chen Zhang, Guang-Sheng Lei. Indiana University School of Medicine, Indianapolis, IN.

Background: Obliterative bronchiolitis (OB) is the leading cause of graft failure and death after lung transplantation. The diagnosis of $\mathrm{OB}$ can be challenging even among experienced pathologists. No effective biomarker or treatment for OB is available. Epithelial-to-mesenchymal transition (EMT) plays critical roles in the pathogenesis of
OB. STIM1 (stromal interaction molecule 1), an endoplasmic reticulum $\mathrm{Ca}^{2+}$ sensor and a direct target of miR-185, has been shown to promote EMT in cancer cells. We hypothesized that the miR-185-STIM1 axis is dysregulated during OB

Design: Fifteen patients who underwent second lung transplantation due to $\mathrm{OB}$ in our institution from 2005-2015 were included as our study objects. The sections of explant lung were examined microscopically and a block showing typical OB lesion was selected from each patient. Normal appearing lung tissue from age and sex matched group of 15 patients was used as control group. The study has been approved by the Institutional Review Boards. The STIM1 immunohistochemical stain was performed on a 4-mm microscopic slide cut from the above mentioned blocks. Total RNA was isolated from formalin-fixed paraffin-embedded (FFPE) lung tissue and was reverse transcribed into cDNA. The expression levels of miR-185 were analyzed by real-time PCR, and compared between the two groups using unpaired student's t-test.

Results: STIM1 was overexpressed in airway epithelium of the lungs with OB, compared with the normal airway epithelium that expressed no or low level of STIM1. The expression level of miR-185 was significantly decreased in lungs with OB compared with that in normal lungs $(\mathrm{P}<0.0001)$

Conclusions: The EMT-related miR-185-STIM1 axis was dysregulated in the lungs with OB, with STIM1 overexpression and miR-185 down-regulation. The dysregulated miR-185-STIM1 axis may serve as a candidate biomarker for diagnosis and a target for therapies.

1944 Comparison of Mutational Profiles in Cytology and Corresponding Surgical Specimens from Patients with Lung Adenocarcinoma

Hamid Zia, Alan Marcus, Brian E Geraghty, Rana S Hoda, Navneet Narula, Hanna Rennert, Helen Fernandes. New York Presbyterian Hospital-Weill Cornell Medical College, New York, NY.

Background: Recent data suggests that surgical specimens from patients with Lung Adenocarcinoma produce a more consistently viable tumor sample for molecular analyses, than cytology cell blocks. Minimal tumor material available in cytology cell blocks is often a limiting factor for multigene molecular assays. We compared mutational profile of cytology and corresponding surgical specimens from patients with Lung Adenocarcinomas.

Design: 16 patients ( 8 female, 8 male; mean age 68 years) with a diagnosis of Lung Adenocarcinoma on cytology (cell blocks) and surgical specimens from same anatomic site were tested. DNA was extracted from the selected tumor tissue and subjected either to targeted Next Generation Sequencing (NGS) using the 50-gene hotspot panel or a realtime PCR assay for detection of $E G F R$ and $K R A S$ variants. A two tailed student t-test was used for statistical analysis.

Results: A specific diagnosis of lung adenocarcinoma was rendered in $83 \%$ of the FNA cases and immunostains for TTF-1 were positive in these cases. The average percentage of neoplastic cells and extracted DNA concentration for cytology specimens was significantly lower than the surgical specimens [Neoplastic cells: $31 \%$ vs $68 \%$ $\left({ }^{*} \mathrm{p}<0.0001\right)$; DNA concentration: $5.5 \mathrm{ng} / \mathrm{ul}$ vs $58.8 \mathrm{ng} / \mathrm{ul}\left({ }^{*} \mathrm{p}=0.025\right) .6$ KRAS and 4 sensitizing $E G F R$ variants were detected in cytology and corresponding surgical specimens from 10 patients. 5 cases were negative for variants in both specimen types. One surgical specimen had a $K R A S \mathrm{Q} 61 \mathrm{H}$ variant that was absent in the corresponding cytology specimen. This discordant specimen had two surgical blocks tested, with the other block giving an identical result to cytology. 6 additional variants $(T P 53=2$; $P I K 3 C A=1 ; A P C=2 ; S T K 11=1$ ) were identified in cytology; corresponding surgical specimens had 8 variants including $T P 53=2 ; P I K 3 C A=2 ; A P C=2 ; S T K 11=1$ and $C D K N 2 A=1)$

\begin{tabular}{|l|l|l|l|l|l|l|}
\hline & $\begin{array}{l}\text { Ave Neoplastic } \\
\text { cells (\%) }\end{array}$ & $\begin{array}{l}\text { Ave DNA } \\
\text { conc. (ng/uL) }\end{array}$ & $\begin{array}{l}\text { \# of EGFR } \\
\text { variants }\end{array}$ & $\begin{array}{l}\text { \# of KRAS } \\
\text { variants }\end{array}$ & $\begin{array}{l}\text { Addt'l } \\
\text { variants }\end{array}$ & $\begin{array}{l}\text { Total \# of } \\
\text { variants }\end{array}$ \\
\hline Cytology & 31 & 5.5 & 4 & 6 & 6 & 16 \\
\hline Surgical & $68^{*}$ & $58.8^{* *}$ & 4 & 7 & 8 & 19 \\
\hline
\end{tabular}

Overall there was $91 \%$ concordance rate among actionable variants and $>85 \%$ correlation among all variants identified in both specimen types.

Conclusions: Despite significant differences in the percentage of neoplastic content and DNA concentration, the NGS results from cytology are comparable to surgical specimens. Cytology specimens are excellent candidates for interrogation of genomic targets with therapeutic significance in patients with lung adenocarcinoma.

\section{Quality Assurance}

1945 Cost-Benefit Analysis of C4d Immunoflourescence and Immunohistochemistry in Evaluation of Pulmonary Allograft Dysfunction Monica Aldulescu, Razvan Lapadat, Robyn Essendrop, Kamran M Mirza, Swati Mehrotra, Vijayalakshmi Ananthanarayanan. Loyola University Medical Center, Maywood, IL.

Background: Antibody-mediated rejection (AMR) is a well-recognized cause of allograft dysfunction in solid organ transplants of the kidney and heart, however no consensus definition exists on its occurence in lung transplantation. Detection of the specific complement degradation product $\mathrm{C} 4 \mathrm{~d}$ by immunofluorescence (IF) or immunohistochemistry (IHC) has shown to be a sensitive and specific marker for AMR; however, its role in evaluating pulmonary allograft rejection remains contentious. The aim of this study is to determine the frequency and clinical impact of C4d IF and IHC and to determine a cost-benefit analysis for performing these tests in lung allograft recipients. Design: Cases of transbronchial lung transplant biopsies were identified using a retrospective review of our database from 2012 to 2015. A total of 194 cases in 104 